International Journal of Modern Physics B

Vol. 31, No. 14 (2017) 1750205 (75 pages)

(C) The Author(s)

DOI: $10.1142 /$ S0217979217502058

\title{
Isolating lattice from electronic contributions in thermal transport measurements of metals and alloys above ambient temperature and an adiabatic model
}

\author{
Everett M. Criss* \\ Panasonic Avionics Corporation, \\ Lake Forest, CA 92630, USA \\ ecriss@eng.ucsd.edu \\ Anne M. Hofmeister ${ }^{\dagger}$ \\ Department of Earth and Planetary Sciences, \\ Washington University, St. Louis, MO 63130, USA \\ hofmeist@wustl.edu
}

Received 27 November 2016

Revised 25 April 2017

Accepted 27 April 2017

Published 15 May 2017

\begin{abstract}
From femtosecond spectroscopy (fs-spectroscopy) of metals, electrons and phonons reequilibrate nearly independently, which contrasts with models of heat transfer at ordinary temperatures $(T>100 \mathrm{~K})$. These electronic transfer models only agree with thermal conductivity $(k)$ data at a single temperature, but do not agree with thermal diffusivity $(D)$ data. To address the discrepancies, which are important to problems in solid state physics, we separately measured electronic (ele) and phononic (lat) components of $D$ in many metals and alloys over $\sim 290-1100 \mathrm{~K}$ by varying measurement duration and sample length in laser-flash experiments. These mechanisms produce distinct diffusive responses in temperature versus time acquisitions because carrier speeds $(u)$ and heat capacities $(C)$ differ greatly. Electronic transport of heat only operates for a brief time after heat is applied because $u$ is high. High $D_{\text {ele }}$ is associated with moderate $T$, long lengths, low electrical resistivity, and loss of ferromagnetism. Relationships of $D_{\text {ele }}$ and $D_{\text {lat }}$ with physical properties support our assignments. Although $k_{\text {ele }}$ reaches $\sim 20 \times k_{\text {lat }}$ near $470 \mathrm{~K}$, it is transient. Combining previous data on $u$ with each $D$
\end{abstract}

This is an Open Access article published by World Scientific Publishing Company. It is distributed under the terms of the https://creativecommons.org/licenses/by/4.0Creative Commons Attribution 4.0 (CC-BY) License. Further distribution of this work is permitted, provided the original work is properly cited.

*E. M. Criss is an employee of Panasonic Avionics Corporation, but prepared this paper independent of his employment and without use of information, resources, or other support from Panasonic Avionics Corporation.

$\dagger$ Corresponding author. 


\begin{abstract}
provides mean free paths and lifetimes that are consistent with $\sim 298 \mathrm{~K}$ fs-spectroscopy, and new values at high $T$. Our findings are consistent with nearly-free electrons absorbing and transmitting a small fraction of the incoming heat, whereas phonons absorb and transmit the majority. We model time-dependent, parallel heat transfer under adiabatic conditions which is one-dimensional in solids, as required by thermodynamic law. For noninteracting mechanisms, $k \cong \Sigma C_{i} k_{i} \Sigma C_{i} /\left(\Sigma C_{i}^{2}\right)$. For metals, this reduces to $k=k_{\text {lat }}$ above $\sim 20 \mathrm{~K}$, consistent with our measurements, and shows that Meissner's equation ( $\left.k \cong k_{\text {lat }}+k_{\text {ele }}\right)$ is invalid above $\sim 20 \mathrm{~K}$. For one mechanism with multiple, interacting carriers, $k \cong \Sigma C_{i} k_{i} /\left(\Sigma C_{i}\right)$. Thus, certain dynamic behaviors of electrons and phonons in metals have been misunderstood. Implications for theoretical models and technological advancements are briefly discussed.
\end{abstract}

Keywords: Thermal diffusivity; metals; electrons; phonons; lifetimes; thermal conductivity; mean free path; high temperature; adiabatic approximation.

PACS numbers: 65.40.G-, 44.05.te, 66.10.cd, 72.10.Bg, 72.15.Eb, 72.15.Lh, 72.60.tg

\title{
1. Introduction
}

In metals and alloys, heat can be conducted by both electrons and lattice vibrations..$^{1-\underline{3}}$ Because electron-phonon interactions occur when heat is conducted through a metal, as evidenced in thermoelectric power ${ }^{4}$ as well as during resistance heating, studying this phenomenon has played an important role in historic and modern investigations of the physics of metals. $\frac{3-\underline{5}}{}$ Electrons have a very low heat capacity but high speeds (near the Fermi velocity) which are thought to compensate during heat transfer. Both properties stem from the Pauli exclusion principle, which only allows electrons near the Fermi energy to be promoted upon receipt of small amounts of heat energy. $\frac{4,6,7}{}$ Phonons, being bosons, are not subject to this restriction, and contain the majority of the heat content of the metal, but move comparatively slowly, similar to sound speeds. ${ }^{8}$ Electronic and phononic processes should be largely independent. ${ }^{2-8}$ Ultrafast optical spectroscopic experiments on thin metal films ${ }^{9}$ and surfaces (e.g., Ref. 10) confirm this deduction. Furthermore, these experiments show that a hot electron gas will equilibrate internally prior to equilibration with the lattice in diverse types of metals. ${ }^{11-14}$ Although the consequences of the electron temperature exceeding the lattice temperature by hundreds of degrees during these transient excitation experiments ${ }^{15,16}$ have received considerable attention, ${ }^{14,17}$ ramifications of this discovery on heat transport in bulk metals at ordinary temperatures (above $\sim 100 \mathrm{~K}$ ) have been overlooked. This paper quantifies the behavior of electrons and phonons in metals and alloys during timedependent heat transport, thereby providing important constraints for theoretical models and for technological applications.

Several parameters (thermal diffusivity, $D$; thermal conductivity, $k$; and heat capacity, $C$ ) describe heat flow through a material. However, previous models and measurements have focused on thermal conductivity, stemming from $k$ being the sole physical property in Fourier's law and thus being more applicable pragmatically. In one dimension,

$$
q=-k \partial T / \partial z
$$


where $q$ is the heat flux, $z$ is the distance, and $T$ is the temperature. Electrons are considered to dominate heat conduction in most metals. ${ }^{2} \underline{-8}$ However, experimental data and theoretical analyses of $k$ show that the phononic (lattice) component is substantial in many transition metals, $\frac{18}{2}$ particularly iron, $\frac{19}{,}$ and roughly equals the contribution from electrons in impure metals or disordered alloys. .5 Ambiguities exist because proportions of the lattice (lat) and electronic (ele) contributions to $k_{\text {meas }}$ have been derived from models ${ }^{5}$ rather than by experimentally isolating either of these components. Section 2.1 describes these models. Here we illustrate significant inconsistencies by comparing the nearly-free electron model to existing measurements of $k$ and $D$, and then explain these inconsistencies in Sec. 1.1 in view of lifetimes ascertained independently via ultrafast spectroscopy. ${ }^{14}$

A simple analysis of available data indicates that problems exist when considering electrons as the important carrier, as follows. If electrons dominate heat transport, then

$$
k_{\text {meas }} \cong k_{\text {ele }}=\rho c_{\text {ele }} D_{\text {ele }},
$$

where $\rho$ is the metal density and $c$ is the specific heat on a per mass basis. All models rest on the mean free gas description of electron transport:

$$
k_{\text {ele }} \cong \frac{1}{3} u_{\text {ele }}^{2} \tau_{\text {ele }} \frac{N}{V} C_{V, \text { ele }}^{*}=\frac{1}{3} u_{\text {ele }} \lambda_{\text {ele }} \frac{N}{V} C_{V, \text { ele }}^{*},
$$

where $u=$ particle velocity, $\tau=$ the mean lifetime, $\lambda=$ mean free path, $N=$ the number of electrons in a relevant volume, $V$, and * indicates a per mole basis. In the long-standing Wiedemann-Franz (WF) law, lifetimes are obtained from the DC conductivity $(\sigma)$ :

$$
\sigma=\frac{N}{V} e^{2} \frac{\tau_{\text {ele }}}{m_{e}}
$$

where $e$ is the electron charge and $m_{e}$ is the electron mass. Using Fermi-Dirac statistics for electron speed and heat capacity provides $k_{\text {ele }}$ with Sommerfeld's ${ }^{20}$ Lorenz number $\left(L_{S}\right)$ :

$$
k_{\text {ele }}=\frac{\pi^{2}}{3} \frac{k_{B}^{2}}{e^{2}} \sigma T=L_{S} \sigma T,
$$

where $k_{B}$ is Boltzmann's constant. To obtain $D_{\text {ele }}$ near room temperature, we combine Eqs. (2) and (3), utilizing

$$
C_{\text {ele }}^{*}=\gamma T=\frac{\pi^{2}}{2} \frac{R_{\mathrm{GC}} Z}{T_{F}},
$$

where $R_{\mathrm{GC}}$ is the gas constant, $Z$ is the valence, and $T_{F}$ is the Fermi temperature. ${ }^{4}$ This representation of $C_{\text {ele }}^{*}$ is advantageous because Sommerfeld's constant $(\gamma)$ does not vary much per mole, ${ }^{21}$ but requires incorporating atomic mass $(M)$ :

$$
D_{\text {ele }}=\frac{L_{S} \sigma T}{\rho c_{\text {ele }}}=\frac{L_{S} \sigma M}{\rho \gamma} .
$$




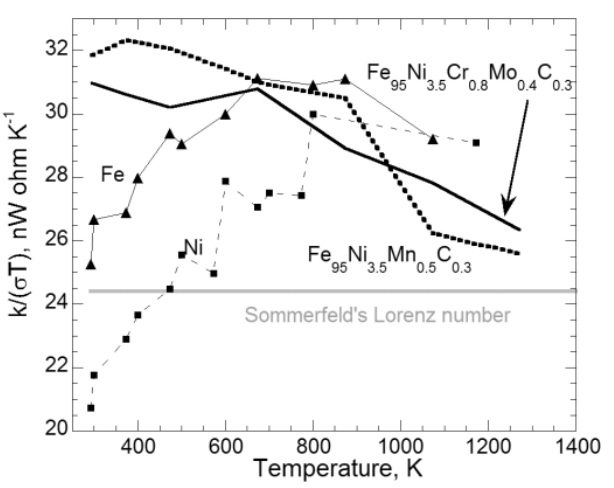

(a)

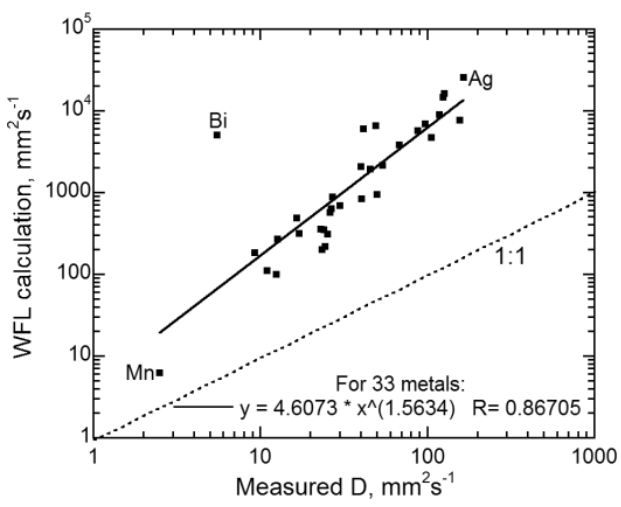

(b)

Fig. 1. Wiedemann-Franz law versus measurements: (a) Thermal conductivity (5), showing that the Lorenz number, $k /(\sigma T)$, depends on temperature, which is unexpected. Triangles $=$ nearlypure $\mathrm{Fe}$, squares $=\mathrm{Ni}$, heavy lines $=$ two different steels, and Gray $=$ Sommerfeld's Lorenz number. Data from Ref. 23. (b) Thermal diffusivity, showing the WF model, Eq. (7), as a function of measurements. Data on $D$ from the present study and Ref. 22, with electrical conductivity from Ref. 24, describing 33 metallic elements. A few elements are labeled.

Measured $k$ (see Refs. 22-24) agrees with the model near $298 \mathrm{~K}$, as is well known. However, as shown in Fig. 1, two problems exist with this electronically dominated model. First, problems exist in describing the temperature dependence of $k$ (e.g., Refs. 4 and 25). Second, measurements of $D$ for bulk metals near $298 \mathrm{~K}$ depart greatly from the nearly-free electron model, should electrons dominate.

\subsection{Misunderstood aspects of heat transport as revealed by ultrafast spectroscopy}

Spectroscopic experiments utilizing femtosecond (fs) laser pulses have revealed that electron-electron scattering events are more probable than electron-phonon events in metals and that thermal disequilibrium exists between electrons and phonons. These findings are supported by theoretical investigations (reviewed by Refs. 14 and 17). Hence, the long-standing descriptions of heat flow across large samples and bulk materials, which argue that electron-phonon scattering dominates and equilibrium exists (e.g., Refs. 2 and 8), need to be reconsidered.

These spectroscopic experiments create a high electron flux by stimulating electrons occupying a broad range of energy levels to higher energies using highintensity, monochromatic light (usually visible), and then monitor the short-time response. ${ }^{12,14,26,27}$ Most experiments are on thin films, although reflectance configurations permit study of single crystals. $\frac{11}{}$ Lifetimes from spectroscopy describe the duration that the excited electrons remain "hot." Thermalization is rapid, $\sim 1$ fs. Next, the photoexcited electrons reequilibrate with each other in $\sim 10-500 \mathrm{fs}$, depending on the material, the thickness of the film, and the energy of the electrons above the Fermi level. ${ }^{14,26}$ Electron lifetimes associated with energies in excess of 


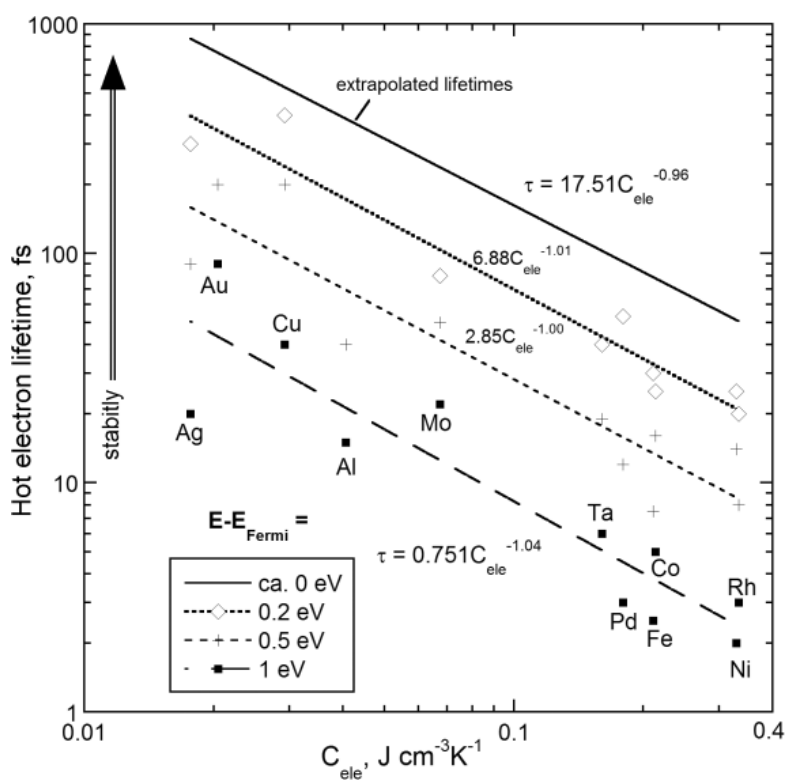

Fig. 2. Dependence of excited electron lifetimes from ultrafast spectroscopy on the electronic heat capacity. Data on lifetimes represent multiple studies of each substance, and were taken from graphs in Ref. 14. Lifetimes for very small energy excesses above the Fermi level were obtained by extrapolating the experimental values. Data on $C_{\text {ele }}$ from Ref. 21. Inverse fits exist whether the basis for $C_{\text {ele }}$ is per mass, per mole, or per volume. In all cases, the correlation coefficients, $R$, vary from 0.73 to 0.99 .

the Fermi level depend on $C_{\text {ele }}$ of the metal (Fig. 2), which is related to the proportion of the electron population that can be excited. Reequilibration with the lattice at ambient temperature occurs much later, in $\sim 1-10$ ps. ${ }^{9,17,26}$

That electron-phonon scattering is the least probable event is emphasized by the behavior of magnetic materials such as $\mathrm{Ni}$, wherein magnetic alignment of electrons occurs after thermal equilibration of the electron cloud, but before the electrons reequilibrate with the lattice. As such, these magnetic interactions involve lifetimes similar to phononic interactions for transition elements, 12 although magnetic lifetimes in the complex pnicnides are comparable to electron-electron lifetimes. 11

Femtosecond spectroscopy (fs-spectroscopy) experiments provide a consistent and coherent picture of the transient behavior of electrons in metals during heat transfer. Absorption of heat (light with frequencies determined by a Planck curve) by some section of a metal causes this portion to exist in a state of quasi-equilibrium, in which temperature differences within the electron gas are rapidly removed, while significant differences between the temperatures of the electron and phonon gases persist for a much longer time.

Disequilibrium of electrons and phonons during transient heating events, as demonstrated by ultrafast spectroscopy, is a consequence of electrons and phonons obeying different statistics, of Coulombic interactions between electrons being 
strong, 4,8 and of electrons moving $10^{3}$ faster than phonons. Disequilibrium during heating is thus compatible with the nearly-free electron model.

\subsection{Ambiguities in the WF model}

Previous assignment of electrons as the main carrier of heat in metals rests on the historic WF law. Yet, agreement of $k$ computed from the WF law with thermal conductivity data occurs only at one temperature [Fig. 1(a)]. This limited agreement suggests that resistivity is not the only factor governing the mean free path. Crucially, the WF law is inherently ambiguous. Its simplicity $(k \propto \sigma)$ results from factors in the product $u_{\text {ele }} C_{\text {ele }}$ canceling. However, factors cancel regardless of whether the quantum product $\left(u_{\text {ele }} C_{\text {ele }}\right)$ or the classical product $\left(u_{\text {gas }} C_{\text {gas }}\right)$ is used. ${ }^{4}-\underline{7}$ Inherent ambiguity is underscored by the historic derivation of Drude, 28 who applied classical statistics to electrons, resulting in nearly the same formula as Eq. (5). Ambiguous behavior is a characteristic of models which contain lumped (multiplied) factors, whereby data can be fit even if the physics is incorrect. ${ }^{29}$

This issue extends beyond uncertainties in the current description of electronic heat flow. Importantly, the $u_{\text {gas }} C_{\text {gas }}$ term is a commonly used representation of $u_{\text {lat }} C_{\text {lat }} \cdot \stackrel{4,6-8}{-8}$ Thus, the WF could actually be describing phonon-phonon scattering, if resistivity is relevant to this mechanism (next subsection).

\subsection{Overlooked implications for electron-phonon interactions in metals}

Although it may seem counterintuitive, the mean free path in phononic transport involves electrical resistivity. This deduction is based on the Born-Oppenheimer (adiabatic) approximation, in combination with electrons and phonons following different statistics: During steady-state, the fast conduction electrons nimbly adjust to the slow motions of the cations ${ }^{4,6-} \underline{-8}$ and hence, the vibrations set up an alternating microcurrent amongst the conduction electrons. No electrical resistance is associated with this AC microcurrent for materials in thermal equilibrium. However, this picture is altered during time-dependent, transient heat flow, as a consequence of thermal disequilibrium between electrons and phonons coupled with their different speeds, as follows:

Electrons move at speeds $10^{3}$ times those of phonons and reequilibrate with one another much faster (up to $\times 10^{3}$ ) than they equilibrate with the lattice. ${ }^{14}$ Applying heat to one end of a metal supplies a flux of photons. The incoming photons excite some electrons but many phonons, in accordance with the statistics of the available states and the photon frequencies. Both excited states travel down the temperature gradient. The front of propagating electrons outpaces the phonons in their respective journeys towards the cold end (see Sec. 2.2 for discussion of how carrier speed relates to $D$ ). By the time that the phonons reach any given region, the electrons have already equilibrated with each other and possess the random motions of a gas. The specific temperature of the electron gas, and whether 
reequilibration with the lattice has occurred when the phonons arrive is immaterial. What is important is that the electrons possess random motions. Thus, in the frame of reference of the forward scattered phonons, the electrons are moving, albeit backwards. Effectively, heat being transported by phonon scattering superimposes an additional DC component on to the preexisting AC electric field.

Conduction of heat by the lattice therefore is tied to electrical resistivity, and, hence, a lattice mechanism can explain the observed connection of bulk heatds transport in metals with electrical resistivity at a single temperature.

In contrast to phononic behavior, inconsistencies exist with a purely electronic mechanism for heat transport. In an imposed electrical field, conduction electrons flowing together in a current do not impede each other; instead, their flow is impeded by vibrating cations (the valance electrons): this impedance is represented by resistivity. Hence, higher probability electron-electron scattering does not involve resistivity, and does not describe key aspects of measured heat transport in bulk metals, as shown in Fig. 1. As such, the WF law can only reasonably describe electronic transport if the electron-phonon mechanism dominates, which is questionable theoretically, as follows:

Unlike electron-phonon scattering, electron-electron scattering should have a high probability due to Coulombic forces being strong., ${ }^{4,6}$ To justify the order of probability associated with the WF law, which is inconsistent with recent ultrafast spectroscopic experiments, ${ }^{9-17,26}$ it was previously argued that the excited electron lacks a state to fall into during electron-electron collisions. $\stackrel{4,6}{ }$ This picture is incorrect regarding heat transfer because it treats electron population statistics as independent of temperature. Clearly, the statistical distribution of states in the "hot" region differs from that of the "cold." The energy levels do not exactly match, including near the Fermi level [Fig. 3(a)], so the transition is much more complicated than the textbook picture of two electrons simply trading places, while being highly probable. In essence, when a "hot" conduction electron travels down the thermal gradient, its electric field distorts the fields of the thermally stable conduction electrons as it enters a colder region of the metal. During a "collision," the interacting electrons form a metastable state [Fig. 3(a)] that has some finite lifetime. After this event, electronic temperature is higher in the region of interaction [Fig. 3(a)].

Improbability of energy transfer without an available destination state for the electron actually applies to electron-phonon scattering. This type of interaction reduces the energy of the carrier electron, but because this energy is lost to a phonon, the surrounding field of electrons will remain mostly unchanged, and no additional destinations will become available. This type of inelastic interaction could be aided near a defect, grain boundary, or impurity atom that provides additional energy levels [Fig. 3(b)]. For example, consider a high purity metal. A metal that is $99.99 \%$ pure has one impurity ion per 10,000 majority ions. With an average interatomic distance of $0.5 \mathrm{~nm}$, this corresponds to one impurity atom in a volume of 1250 common cations, providing a distance $\sim 600 \mathrm{~nm}$ between impurity atoms, 


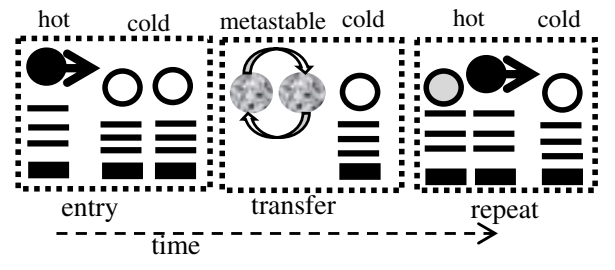

(a)

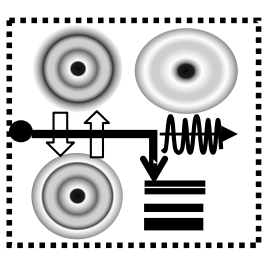

(b)

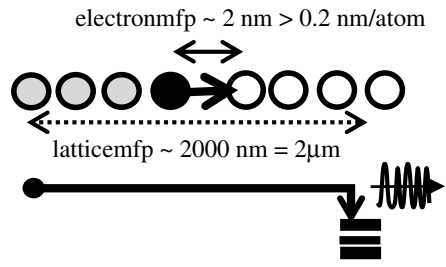

(c)

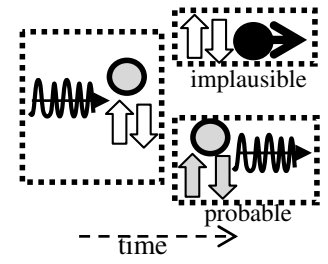

(d)

Fig. 3. Schematics of electron and phonon behaviors in metals. In all panels: dotted outline $=\mathrm{a}$ small volume of the metal in a temperature field; dashed arrow indicates time; various small circles $=$ individual electrons in different states; horizontal lines = energy levels associated with the region of space where the electron of interest resides; large shaded circles $=$ atoms; pairs of open arrows indicate atomic motions; thick black arrow = electron motion down the thermal gradient and termination of motion; thin arrow with squiggle $=$ phonon motion. (a) Sequence of events during electron-electron scattering. Left panel = initial state. At the hot end (left), a conduction electron (black dot) is excited above the top of the Fermi levels (horizontal bars) and travels towards the cold end (right), where small proportion of the electrons (circles) are near the top of their Fermi levels, which differ from the levels in the hot region. Middle panel $=$ thermalization of conduction electrons. In order for the "hot" and "cold" electrons to equilibrate, they interact (stippled circles and curved white arrows). Energy levels are not shown during this brief, metastable state. Right panel $=$ progression of heat across the volume. Through the interaction, the incoming excited electron has lost some of its energy, and is now part of the equilibrium, but hot, state (gray circle). This energy was transferred to the middle electron (black dot) which then travels into the colder region. Repetition of these steps (i.e., thermal diffusion) brings the electron population across the metal into the hot state. (b) Electron-phonon interactions. Conduction electrons avoid the vibrations of the cations, but if a defect is encountered, and an open energy level exists, then the incoming hot electron can give energy to the phonons. Impurities and point defects have separations consistent with mean free paths attributed to phononic interactions, see text. The excited phonon (wave with light arrow) next travels into the cold region, but slowly. (c) Comparison of mean free paths, calculated using a typical Fermi velocity $\times$ lifetimes from thin film spectroscopy. 14 The exchange of energy between electrons (top row) occurs at about every $2 \mathrm{~nm}$ and at $u_{\mathrm{fermi}}$. This distance is similar to the average distance of $2.3 \mathrm{~nm}$ for $1 \%$ of the conduction electrons being near the top of the Fermi level (suggested by comparing quantum to classical heat capacities). Bottom row shows the exchange of energy of electrons with the lattice being over distances $\sim 1000 \times$ longer ( $p$ s rather than fs lifetimes ${ }^{14}$ ). After this exchange, a phonon is excited. (d) Movement of heat by phonons. Left panel $=$ the initial state where a phonon enters some volume in the metal. Due to the relative speeds, the electron population has already transformed to the hot state (gray circle), but the lattice is in the cold state (white arrows). Two different exchanges are possible: Top right panel $=$ excitation of an electron, which increases the temperature of the electron gas, which is already hot, while leaving the lattice in the cold state. Bottom right panel $=$ excitation of another phonon, where the lattice temperature increases to the hot state. The electron giving energy to a phonon is irreversible, because it advances the thermal fields toward equilibration. 
roughly. For typical Fermi velocities, this corresponds to a lifetime $\sim 1$ ps, which is considered to represent reequilibration of the electrons with the lattice. Point or line defects, which should have roughly the same spacing as impurity atoms, will provide roughly similar lifetimes. Regardless of the details, ultrafast optical spectroscopy shows that electron-phonon interactions are far less probable than electron-electron scattering [Fig. 3(c)], and in some cases, such as the complicated pnicnides, $\frac{11}{1}$ it is significantly less probably than magnetic processes.

The behavior of a hot electron after it undergoes a "collision" is very important. If the collision is with an electron near the Fermi level, the heat can be propagated [Fig. 3(c)]. If instead, some hot electron interacts with the lattice, then this electron's thermal energy is lost to the lattice, becoming part of the phononic heat transfer mechanism at slow acoustic speeds [Figs. 3(b) and 3(c)]. If the diffusional sequence for electron transport in a metal is electron-phonon collisions followed by a phonon-electron event [Fig. 3(d), top], then the associated speed cannot be $u_{\text {Fermi }}$ but instead lies between electronic and phononic speeds, depending on event probabilities. Moreover, a serious problem exists with scattered phonons exciting electrons during heat transfer across a material, as follows.

Thermodynamic law dictates that the phonon donating heat must be at a higher effective temperature than that of the receiving electron. Because phonons move slower than electrons, the phonon excited by an electron travels into an area where the electron temperature is higher than the lattice temperature (viewing both as a distribution of temperature and energies, and recognizing that electrons precede phonons during travel away from the heat source). These stipulations make inelastic phonon-electron collisions unlikely to contribute to thermal diffusivity. The much higher probability event is phonon-phonon scattering, which elevates the lattice temperature [Fig. 3(d), bottom].

Given the above arguments, heat transfer in bulk metals should be dominated by phonon-phonon scattering. To explore this possibility, we consider the end-member case:

$$
k_{\text {meas }} \cong k_{\text {lat }}=\rho c_{\text {lat }} D_{\text {lat }} \cong \rho c_{\text {meas }} D_{\text {meas }},
$$

and compare measured $D$ and $k$. Excellent agreement exists at 298 K (Fig. 4), and at other temperatures, as is known. ${ }^{22,24}$ Importantly, excitable phonons greatly outnumber excitable electrons (those with near the Fermi level), and hence phonons uptake the vast majoring of heat [Infrared (IR) light] entering the solid. Because phonons contain the heat content of the metal, only their interactions can propagate significant amounts of heat. The simplest explanation of heat transfer in metals is phonon scattering.

Historical assignment of the heat carrier as electrons resulted from lack of data on hot electron lifetimes, and from ambiguities in the WF law. Because the WF law contains lumped parameters which obscure the effect of individual constituents (in this case, $u C$ ) it cannot be used to rigorously determine the effects of any particular component. $\stackrel{29}{ }$ Understanding heat transfer in metals requires new models 


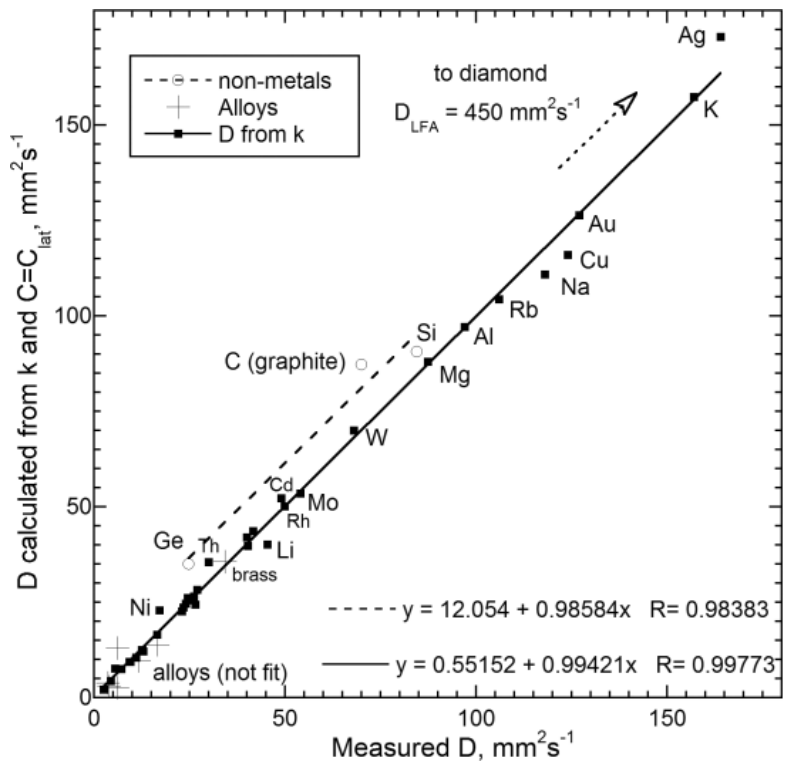

Fig. 4. Dependence of $D$ calculated from measured $k$ using measured heat capacity, which describes the lattice, $\underline{4}$ on direct measurements of $D$. If $D$ had been computed using $c_{\text {ele }}$, which is required if electrons are the dominant carrier, then the computed values would be $100 \times$ those shown. Data on $k$ from Ref. 24. Direct measurements of $D$ from Refs. 33 and 22, describing 39

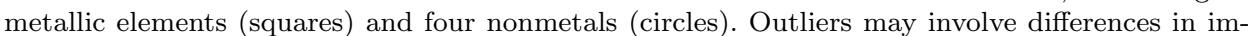
purities, because purification techniques have improved since the data were collected, see text. All semiconductors lie above the 1:1 line because ballistic radiative transfer affects $k$ but was removed from $D$ in the laser-flash analysis (LFA) studies shown. Diamond was not included in the fit due to its extreme ballistic transfer.

and measurements to further investigate and clarify the results provided by ultrafast spectroscopy.

\subsection{Why measuring thermal diffusivity provides additional insights on mechanisms over measuring thermal conductivity or thermal effusivity}

From the above, independent measurement of at least one of the electronic and lattice components in metals is needed to improve our understanding of the microscopic basis of heat diffusion. Previously, different mechanisms were evaluated through modeling measurements of $k$, but such results are ambiguous due to the strong influence of specific heat [Eqs. (2) and (8)] and the lack of temporal resolution. Limitations on measurements of $k$ are shared by thermoreflectance measurements, which extract $k$ from thermal effusivity $(e)$, where $e^{2}=k \rho c=\rho^{2} c^{2} D . \underline{30}$ Although this method probes short times using fast detection systems, experimental conditions are quasi-steady-state, resulting from multiple pulses. As such, thermoreflectance measurements do not record the temporal response to an individual heat pulse. Hence, mechanism proportions from thermoreflectance data ${ }^{31}$ are model- 
dependent, as in existing analyses of $k$ (below and in Sec. 2.1). Furthermore, the influence of heat capacity is even stronger than in measurements of $k$. For the above reasons, and because bulk material properties are the subject of this paper, we focus on other means for measuring $D$ and $k$.

Regarding thermal conductivity, different signs for $\partial k_{\text {meas }} / \partial T$ at high $T$ in crystals and glasses have been interpreted as these material types are having different transport mechanisms. ${ }^{32}$ But, $D$ goes as $F T^{-G}+H T$ regardless of the degree of cation order or crystallinity, and so the difference in $\partial k_{\text {meas }} / \partial T$ stems instead from the relative sizes of $\partial D / \partial T$ and $\partial c / \partial T{ }^{33,34}$ Similarly, $k_{\text {meas }}$ of metals being large compared to $k_{\text {meas }}$ of electrical insulators has been interpreted in terms of different mechanisms operating: however, $k_{\text {meas }}$ in metals is large mainly because metals have high density and fairly high heat capacity. ${ }^{24}$ Comparing values of $D$ provides a contrasting picture. Among the elements, most metals have $D$ of $\sim 3-50 \mathrm{~mm}^{2} \cdot \mathrm{s}^{-1}$ at $298 \mathrm{~K}$, whereas electrically insulating diamond has $D=450 \mathrm{~mm}^{2} \cdot \mathrm{s}^{-1}$ which is triple the $D$ value of the highest metal (Ag); similarly, semiconductors (Ge, graphite, and Si) have $D=25-90 \mathrm{~mm}^{2} \cdot \mathrm{s}^{-1}$, as in moderately efficient metals ${ }^{22,23,33}$ (Fig. 4). Thermal conductivity data, being the product of $D$, density, and heat capacity, each of which respond differently to temperature and structure, provide insufficient evidence that heat transport mechanisms differ between metallic and insulating elements.

Measurements of thermal diffusivity have great potential to improve our understanding of metals. Not only do these measurements isolate the movement of heat from the amount of heat carried, which is portrayed separately by the physical property of heat capacity, but furthermore, diffusivity techniques are transient (time-dependent). Another obvious advantage is that $D$ depends more simply on $T$ than does $k$, given Eq. (2) and the complex dependence of $c_{\text {meas }}=c_{\text {lat }}$ on $T$. More importantly, Eq. (2) and $c_{\text {ele }} \ll c_{\text {lat }}$ requires that $D_{\text {ele }} \gg D_{\text {lat }}$ for all conditions except $k_{\text {ele }} \ll k_{\text {lat }}$, which is unexpected. Fast electron speeds also suggest high $D$ (Sec. 2.2). Much larger $D$ being expected for electrons than for phonons should make these two processes distinct in transient measurements. In detail, $D$ alone governs temperature-time $(T-t)$ evolution:

$$
\frac{\partial T}{\partial t}=D \frac{\partial^{2} T}{\partial z^{2}}
$$

This simple form for the heat equation ${ }^{35}$ describes small changes in $T$, where relevant physical properties $(k, c$, and $\rho$ ) can be treated as temperature-independent. As implied by the form of Eq. (9) and as detailed in Sec. 2.2, fast (high $D$ ) heat transfer processes can be distinguished from slow (low $D)$ in methods which directly measure the time dependence of heat flow across a sample. Mechanisms with different speeds, e.g., photonic and phononic heat transports in insulators, are distinguishable in transient experiments because the speed of the heating front is directly related to carrier speed,,$\frac{36}{}$ see Sec. 2 . 


\subsection{Purpose of the present study}

This paper uses LFA ${ }^{37,38}$ to quantify the temperature dependence individually for $D_{\text {ele }}$ and $D_{\text {lat }}$ for diverse metals at ordinary temperatures, independent of any formulations suggested by mechanistic models. Low $T$ behavior likely differs, but is not addressed here. The LFA method (Sec. 2.2) is robust, yet simple, and, importantly, measures the time-dependent response of temperature to a small excursion from thermal equilibrium. ${ }^{39}$ We explore conditions not previously investigated: long samples over short durations (Sec. 3). We find that most metals, especially those with high $c_{\text {ele }}$ and high IR penetration, $, 40,41$ have a fast, weak diffusive signal in addition to the previously monitored, slow, strong signal. Various tests confirm that the rapid rise is intrinsic. The signal is analyzed using a slight modification of Parker et al.'s ${ }^{37}$ original adiabatic model. ${ }^{38,39}$ Signal properties suggest an electronic mechanism. From our measurements of $D_{\text {ele }}$ (Sec. 4 ), we compute $k_{\text {ele }}$ using previous data on $\rho$ and $c_{\text {ele }},{ }^{21,24}$ and find that measured $k_{\text {ele }}$ considerably exceeds model predictions. This discrepancy traces to errors in the classical physics formulations which we reveal (Sec. 5) by deriving sum rules for thermal conductivity components. Our model conserves energy during transient conditions in parallel heat flow, and suggests revisions in current mechanistic models.

Analyzing our data on $D_{\text {ele }}$ using known carrier speeds gives thermal lifetimes of electron-electron scattering and its temperature dependence (Sec. 6). Attenuation of this signal with sample length is consistent with electron-phonon scattering removing electrons from their thermal current. We further show that magnetism alters the behavior of the electrons during heat transport. Our results agree reasonably well with fs-spectroscopy even though the latter probes higher fluxes and energies of electrons. ${ }^{9,13}$ Mean free paths deduced from previous data on $k_{\text {meas }}, \rho$, $c_{\text {lat }}$, and acoustic velocities for elements at $298 \mathrm{~K}$ (e.g., Ref. 24) and other observations require a lattice mechanism, which is what produces the strong, slow response in LFA measurements. The relationships among electrical and lattice components of thermal conductivity, thermal diffusivity, and heat capacity are understood by considering both the temperature evolution during transient heating and the roles played by carrier speed, mean free path, and absorptivity at infrared frequencies (Secs. 6 and 7).

\section{Background}

\subsection{Problems in previous efforts to extract $k_{\text {lat }}$ and $k_{\text {ele }}$ from measured $k$}

In a thermal gradient, heat is conducted by diffusion because the energies of the particles near the hot end are greater than those near the cold end. Both phonons and electrons diffuse heat in a metal, as first proposed by Koenigsberger. $\cdot$ Modern analyses of bulk thermal conductivity measurements of metals assume that a simple sum pertains ${ }^{3,5,18}$ :

$$
k_{\text {meas }}=k_{\text {lat }}+k_{\text {ele }}
$$


Meissner proposed Eq. (10), see based on fitting a few experimental data points to $k=A+B T^{-1}$, where $\bar{A}$ and $B$ are constants presumed to represent different mechanisms. $\frac{42,43}{}$ A derivation was neither provided in these early works nor subsequently $2,3,5,44,45$ to the best of our knowledge. The lack of an explicit derivation during the crucial period when quantum mechanics was initially applied to the electron prevented critical analysis of Eq. (10) and the limiting conditions under which it operates. Hence, some consequences of electrons and phonons following different statistics have gone unrecognized. Of particular importance is that thermal fields associated with each particle type can differ in accordance to their statistics: for example, in response to high frequency, high intensity laser pulses, electrons are immediately promoted to high energy levels, next they thermalize to a Fermi distribution which may be $\sim 500 \mathrm{~K}$ different from ambient. ${ }^{15,16}$ The electrons may then experience magnetic interactions, before finally reequilibrating with the phonon gas. $\frac{11,13,14,26}{}$ After presenting our results (Sec. 4), conditions under which Eq. (10) is valid will be stipulated (Sec. 5).

The electronic mechanism is thought to dominate metals, based on Eq. (10), because Sommerfeld's ${ }^{20}$ semiclassical expression of the Wiedemann-Franz law (5) gives $k_{\text {ele }}$ similar to $k_{\text {meas }}$ at $298 \mathrm{~K}$. Sommerfeld's derivation accounts for only electrons close to the Fermi level participating, stemming from the Pauli exclusion principle, which results in $c_{\text {ele }}$ for metals being small $\left(\sim 1 \%\right.$ of $c_{\text {lat }}$, except below $\sim 20 \mathrm{~K}) \cdot{ }^{4-7}$ For similar reasons, Fermi velocities $\left(u_{F} \sim 10^{6} \mathrm{~m} \cdot \mathrm{s}^{-1}\right)$ describe electrons participating in heat transfer. ${ }^{4}$ These speeds are $\sim 100$ times faster than acoustic (thermal) velocities (see tables in Refs. 4 and 6). Notably, the WF law assumes that the mean free paths of the electrons are identical in thermal and electrical fields, which is inexact (Ref. 4, p. 323; Secs. 5 and 6).

For many materials, the WF law is reasonably accurate near $298 \mathrm{~K}$, but a temperature-dependent fitting function is needed otherwise: this adaptation is in violation of the underlying assumptions. ${ }^{4}$ Furthermore, disagreements with Eq. (5) are commonly significant. ${ }^{25}$ Figure 1 shows that minor alloying elements in steels affect not only the values of $k /(\sigma T)$ but also the sign of the temperature derivative. Nickel alloys 46 and purple bronze ${ }^{47}$ violate the WF law. For both Fe and Ni, the pressure derivatives of the electrical and thermal conductivities differ, $\frac{48}{}$ also in violation of the WF law. Violations and ad hoc use of a temperature-dependent fitting function suggest that the lattice component is significant. Furthermore, phonons in most metals are limited to the acoustic type, which is the most efficient contributor to phonon-phonon scattering in electrical insulators ${ }^{49}$ and therefore, phononic transport in metals should be substantial. Notably, the element with highest $D$ by far is diamond ${ }^{24,33}$ which only transports phononically. Similarly, $D$ of Si exceeds $D$ of most metals (Fig. 4).

Previously, to decipher the how much each mechanism contributes, data on $k_{\text {meas }}$, which were collected as a function of temperature, have been fit to various dependencies on $T$ (e.g., Ref. 19). Ho et al. ${ }^{18}$ concluded that $k_{\text {meas }}=1 /(B / T+A)$ 
for 10 binary alloy systems where the constant $A$ is related to impurity scattering and constant $B$ stems from the WF law. Different forms for $k$ as a function of $T$ have been derived for pure metals and other alloys. Ewing et al. ${ }^{50}$ fit over 140 metals and alloys to the equation: $k_{\text {meas }}=A T+B T^{2}+F / T$, where $F$ is another constant. Smith and Palmer's ${ }^{51}$ formulation, $k_{\text {meas }}=A+B T$, is widely used. ${ }^{52}$ These recent studies support neither Meissner's fit nor Eq. (10).

Explaining the remarkable variability of $k_{\text {meas }}$ and its $T$ derivative for metals and alloys has engendered complex models. $\frac{5}{}$ That many different models have been posited for thermal conductivity of metals suggests shortcomings exist in the current understanding.

\subsection{LFA: Principles and practice}

The large number of scientific papers dealing with applications and continued developments of the flash method testify to the quality of the underlying principles. $\frac{39}{}$ Many reviews exist. .53 Briefly, in this transient method, temperature is measured as a function of time over a constrained and constant length, and thermal diffusivity is extracted from these raw data using Eq. (9).

In LFA experiments ${ }^{37,39,53}$ emissions are recorded remotely, using an IR detector, from the rear (top) surface of a slab, with length $L$, parallel faces, and area $A$ [Fig. 5(a)]. A heat pulse ( $Q$ is applied remotely to a thin graphite coating on the front (bottom) surface of the slab using a laser or lamp, and the subsequent time-evolutions of the emissions from the front (rear) surface are recorded (Fig. 6). Because small amounts of heat are applied and temperature changes are small,

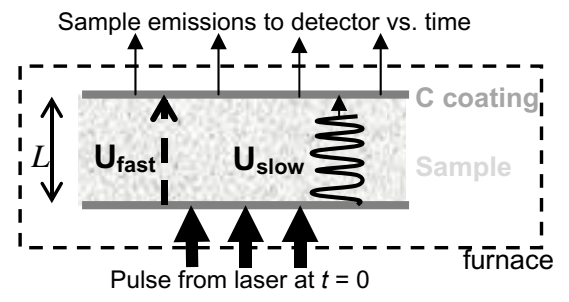

(a)

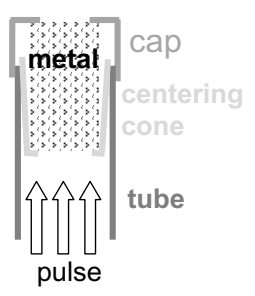

(b)

Fig. 5. Schematics of LFA experiments. (a) Operation essentials. Dashed frame indicates furnace surrounding the sample. Speckled rectangle with thickness $L$ shows the sample edge-on, which has graphite-coated faces. Arrows show receipt of energy from the laser and travel of emissions from sample to detector. Dashed and squiggle arrows inside the sample indicate heat transfer processes traveling at relatively fast and slow speeds. (b) Detailed cross-section of the cylindrical sample and holder. Solids = three graphite parts, distinguished by shade. Stipple = metal sample. Arrows $=$ path of the laser, which is blocked from reaching the detector, which therefore only collects emissions from the faces of the sample and cap. The base of the centering cone may be heated by the laser pulse, but its increase in temperature must be transferred radially through the tube and cap, and then vertically to be sensed. Thermal contacts between the graphite parts and metal are poor so any such conductive transfer is greatly delayed, and cannot possibly produce the rapid rise observed for certain metals. 


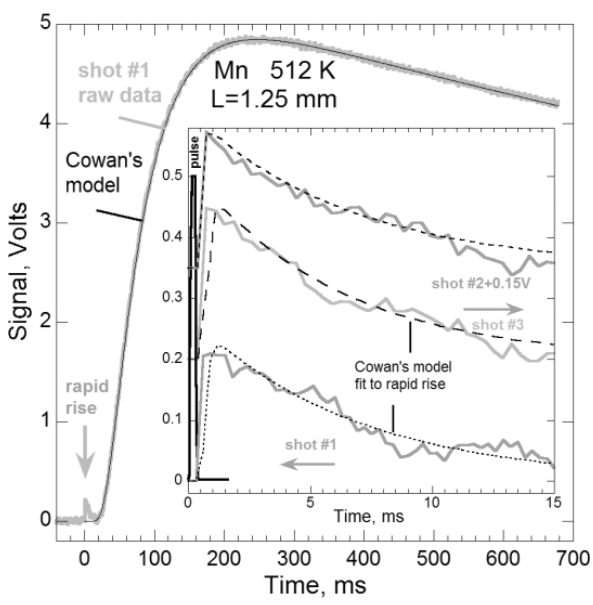

(a)

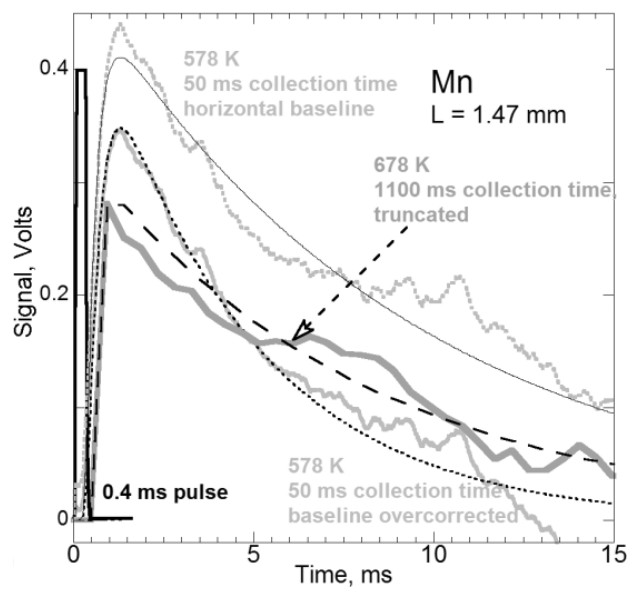

(b)

Fig. 6. Temperature-time curves for thin samples of the element Mn. (a) Mn at $512 \mathrm{~K}$ with $L=$ $1.25 \mathrm{~mm}$. The fits (thin black) to the raw data (thick gray) are over the entire curve (large panel), or only over the rapid rises (inset). Thick black line = laser pulse. Because the slow rise begins at $20 \mathrm{~ms}$ whereas $50 \mathrm{~ms}$ is the shortest collection duration possible with our software, we could not record the rapid rise at better resolution. Nonetheless, the rapid rise differs insignificantly between the three shots acquired under the same conditions. (b) Slightly thicker sample of Mn, comparing data from short and long collection times, using a narrow pulse. Linear baseline corrections have a small effect on most shots. Short shots used a horizontal correction.

emissions are proportional to temperature. ${ }^{38}$ Thus, the raw data are referred to as a $T-t$ curve. Its shape determines the thermal diffusivity in accordance with Eq. (9). For a diffusional process, an asymmetric "S" shape is observed (Fig. 6) which describes heating of the rear (top) surface following the laser pulse, and subsequent reequilibration of the sample with the surroundings.

Various mathematical algorithms exist to address details of the "S" shape. $\frac{54}{4}$ The basic physics is embodied in a simple relationship used in the original (adiabatic) analysis ${ }^{37}$ :

$$
D=0.138785 \frac{L^{2}}{t_{1 / 2}},
$$

where $L$ is the sample length and $t_{1 / 2}$ is half of the time taken for the rear surface to reach maximum temperature, as indicated in Figs. $5(\mathrm{a})$ and 6 . Note that $t_{1 / 2}$ is a measurement time, but is related to much shorter lifetimes as discussed below. Equation (11) comes from a one-dimensional heating model. $\frac{35}{5}$ The numerical factor results from the summations. Because time and distance can be measured accurately, $D$ can be measured accurately.

Advantages of LFA are that neither heat input nor absolute temperature needs be quantified, and physical contacts, which produce heat losses, are avoided. Furthermore, baseline corrections remove effects of stray light and background radiation 
from hot components. Requirements are straightforward $37-39,53$ : It is important to have parallel faces, to avoid strong temperature gradients across the sample, and for the pulse to be absorbed by a thin coating (Fig. 5). Ballistic transmission of light through partially transparent samples added systematic errors up until 1998, $\frac{55}{,}$ but this correctable effect does not apply to the opaque, bulk metals examined here. Similarly, errors associated with finite pulse duration have been removed through modeling. ${ }^{56}$ Improvements in the LFA technique over the 55 years since its development have led to a highly accurate and robust, yet simple, method for evaluating transient heat conduction. $\underline{39,53}$

The rise in temperature (for a single temperature field) is described by ${ }^{37}$

$$
\Delta T \cong \frac{Q}{A L C}
$$

where $C=$ heat capacity on a per volume basis $=\rho c$. The remainder of this paper utilizes $C$. To meet the conditions underlying Eq. (9), $Q$ is kept sufficiently low so that $\Delta T<4^{\circ} \mathrm{C}$. Consequently, conditions are not far from equilibrium. The temperature dependence of $D$ is then determined solely by varying furnace temperature. $37-39,53$ Heat capacity can be determined from $\Delta T$ using additional information on $Q$. But because the efficiency of energy absorption affects $Q$, this determination assumes only one carrier exists. Two carriers will produce different rise heights depending on uptake proportions, carrying capacities, and the processes involved in converting the heat of each carrier to surface emissions. Because the incoming heat (photons) is disparately absorbed by electrons and phonons (due to differing statistics and infrared photons having low energy) and because these carriers are nearly independent, Eq. (12) with $C=C_{\text {lat }}$ describes the phononic uptake, because this carrier absorbs virtually all the power input. Since electrons absorb only a small fraction of the applied heat compared to the greedy phonons, we suggest:

$$
Q_{\text {ele }} \cong \frac{Q C_{\text {ele }}}{C_{\text {lat }}} .
$$

The efficiency of electronic uptake compared to phononic may also factor in.

Under adiabatic conditions, emissions (i.e., T) remain constant for a "long" time after the maximum temperature is reached. More commonly, the detector signal decreases with $t$ after reaching a maximum (Fig. 5) due to the sample cooling radiatively to the surroundings, which requires use of more complicated models than Eq. (11) $.54,55$ For a robust fit to the $T-t$ curves, the experimental duration should be $\sim 10$ half-times. However, very long durations are often associated with a timedependent baseline. Therefore, thin samples are used to provide short collection times when ascertaining low $D$, as in glasses. $\frac{34}{}$ Regarding the contrasting situation of high $D$, the shortest duration available in any given apparatus requires some minimum $L$ for any given $D$. Also, rise times should exceed the $\sim$ ms duration of the purse. Thus, to quantify large $D$, large $L$ is needed [Eq. (6)]. Previous LFA measurements of metals typically probed sections with thickness $L$ of $1-4 \mathrm{~mm}$ 
(Refs. 37 and 57) which provides $T-t$ curves that extend to 10 half-times over a reasonably short time, as in Fig. $6(\mathrm{a})$, and $D$ from $\sim 2 \mathrm{~mm}^{2} \cdot \mathrm{s}^{-1}$ to $\sim 170 \mathrm{~mm}^{2} \cdot \mathrm{s}^{-1}$ which is consistent with other measurements. ${ }^{22,57-60}$ From Eq. (11), different heat transport mechanisms, if these have sufficiently different thermal diffusivities, will be manifested in the temperature-time history as sequentially occurring rises for any given length. This behavior can be explained in terms of a certain speed being associated with the heating front, as follows:

All solutions to Eq. (9) have the form:

$$
D \sim \frac{L^{2}}{\zeta},
$$

where $\zeta$ is a thermalization time (e.g., Ref. 35) which differs from the half-time by a geometrical factor. From dimensional analysis,, 36 the heating front moves at a speed of $u \sim L / \zeta$. Inserting this linear approximation into Eq. (14) gives

$$
u \sim \frac{D}{L}
$$

If it was possible to conduct the experiments over lengths $L$ comparable to the mean free path $(\lambda)$, then $u$ would be the average carrier velocity. High $D$ thus correlates with high carrier speed. For an experiment at a given $L$, mechanisms with substantially different $D$ have different $t_{1 / 2}$ [Eq. (11)], and should be distinct in the $T-t$ curves, so long as interactions between carriers are weak, and the apparatus has sufficient resolution to capture the shorter mechanism. Carrier speeds for electronelectron and phonon-phonon mechanisms differ by $\times 1000$. So, roughly, $D_{\text {ele }}$ should be about $1000 \times D_{\text {lat }}$, and so the two mechanisms should be distinguishable in $T-t$ curves.

Sequential rise times occurring for independent mechanisms is well known in electrical insulators which are partially transparent to high frequency light (e.g., Ref. 55). The carriers for transparent insulators are phonons and high- $\nu$ photons, which have vastly different speeds over any given distance (sound versus light speeds over small distances). A very rapid initial rise is associated with the photons,, 55 whereas the phononic pulse is slow and gradual. One identifying factor of a photonic signal is that its maximum height increases greatly with $T$ (see $T-t$ curves in Ref. 61). Another clue is that the photonic shape is not the characteristic, diffusional "S" following the pulse. Instead, the signal height increases strongly only while the laser pulse is applied: after the pulse, decay is quite rapid as the sample reequilibrates with the surroundings. $\frac{36}{6}$ The photonic increase is not instantaneous because the pulse is gradually applied. But because photons are ultrafast, the time dependence of photonic rise is unaffected by either changing sample length (e.g., from $\sim 0.1 \mathrm{~mm}$ to $10 \mathrm{~mm}$ ) or by temperature: the photonic rise always overlaps with the pulse. Mehling et al.'s model ${ }^{55}$ removes the effect of the rapid initial rise on extraction of $k_{\text {lat }}$ from $T-t$ curves. Applying Eqs. (14) and (15) to $D$ of insulators yields carrier speeds similar to acoustic velocities. $\frac{3 \overline{6}}{.}$ 
The above discussion shows that conduction of heat by (fast) electrons should be distinct from conduction by (slow) phonons and (ultrafast) photons in LFA measurements because of their substantially different arrival times and relative rise heights, as well as the different temperature dependence of these two mechanisms. Importantly, whether a process involves diffusion is evident in the shape of the $T-t$ curves (i.e., in the raw data: Fig. 6). Finally, Eqs. (14) and (15) permit extraction of lifetimes from our data, even though our measurement times are slow compared to the actual physical process. The latter is possible because diffusion consists of repeated microscopic events, as regulated by Eq. (9) and encapsulated in Eq. (15).

\section{Experimental}

\subsection{Samples, sample preparation, and length requirements}

Samples are described in Table 1. Compositions are based on atomic percentages. This collection spans a wide variety of metals and alloys, and includes elements for which IR reflectivity data ${ }^{40,41}$ or $C_{\text {ele }}$ data exist, ${ }^{21}$ or were studied extensively using ultrafast spectroscopy (Fig. 2; Ref. 14).

Regarding Mn, we selected a few pieces with the least amount of surface pits, to minimize the effect of porosity reducing $D$ from intrinsic values. ${ }^{62}$ Otherwise, our metals and alloys are visually homogeneous, although the alloys are generally chemically and structurally heterogeneous on a microscopic scale. This is certainly the case for the hexahedrite. This type of iron meteorite (chemical type IIAB) almost entirely consists of low-Ni $\alpha$-kamacite (bcc with 5-10 wt.\% Ni), but has small amounts of other phases (e.g., FeS, Ref. 63). Hexahedrites are mechanically twinned as evidenced by Neumann lines, which appear in etches and involve lamella of $1-10 \mu \mathrm{m}$ wide by possibly $\sim \mathrm{cm}$ long (typical of North Chile samples). In addition, all iron meteorites have been shocked and are deformed to some degree.

Our Mn-bronze (Table 1 ) is actually a brass and is slightly magnetic. Its magnetism is not described in the literature, but may resemble that of Heusler alloys, $\frac{64}{4}$ which are ternary alloys containing $\mathrm{Mn}$ with fcc structures. Its $\mathrm{Cu}$ content falls near the edge of the $\alpha$-brass field and $\mathrm{Mn}$ and $\mathrm{Al}$ are present, as in other Heusler alloys. The temperature of the Curie transition is unknown. Our experiments do not reveal a Curie transition for this material, although obvious Curie transitions exist in our $\mathrm{Ni}$ and invar data.

For thermal diffusivity measurements, sections were sawed and/or ground into disks with nearly-parallel surfaces of $\sim 8-15 \mathrm{~mm}$ diameter $(d)$ with "standard" 1.25-3.3 mm thicknesses $(L)$ for ordinary $D<150 \mathrm{~mm}^{2} \cdot \mathrm{s}^{-1}$ as well as with longer lengths (4-22 mm) for higher D. Some samples (Mn, SRM-1461) were available only in short lengths. After sand-blasting with 50-150 $\mu \mathrm{m}$ alumina grit, samples were cleaned ultrasonically, and then were immediately sprayed with a thin coating of graphite, roughly of micrometers thickness, to avoid oxidation. For repeated runs, these steps were repeated to provide a clean metal surface for each experiment. 


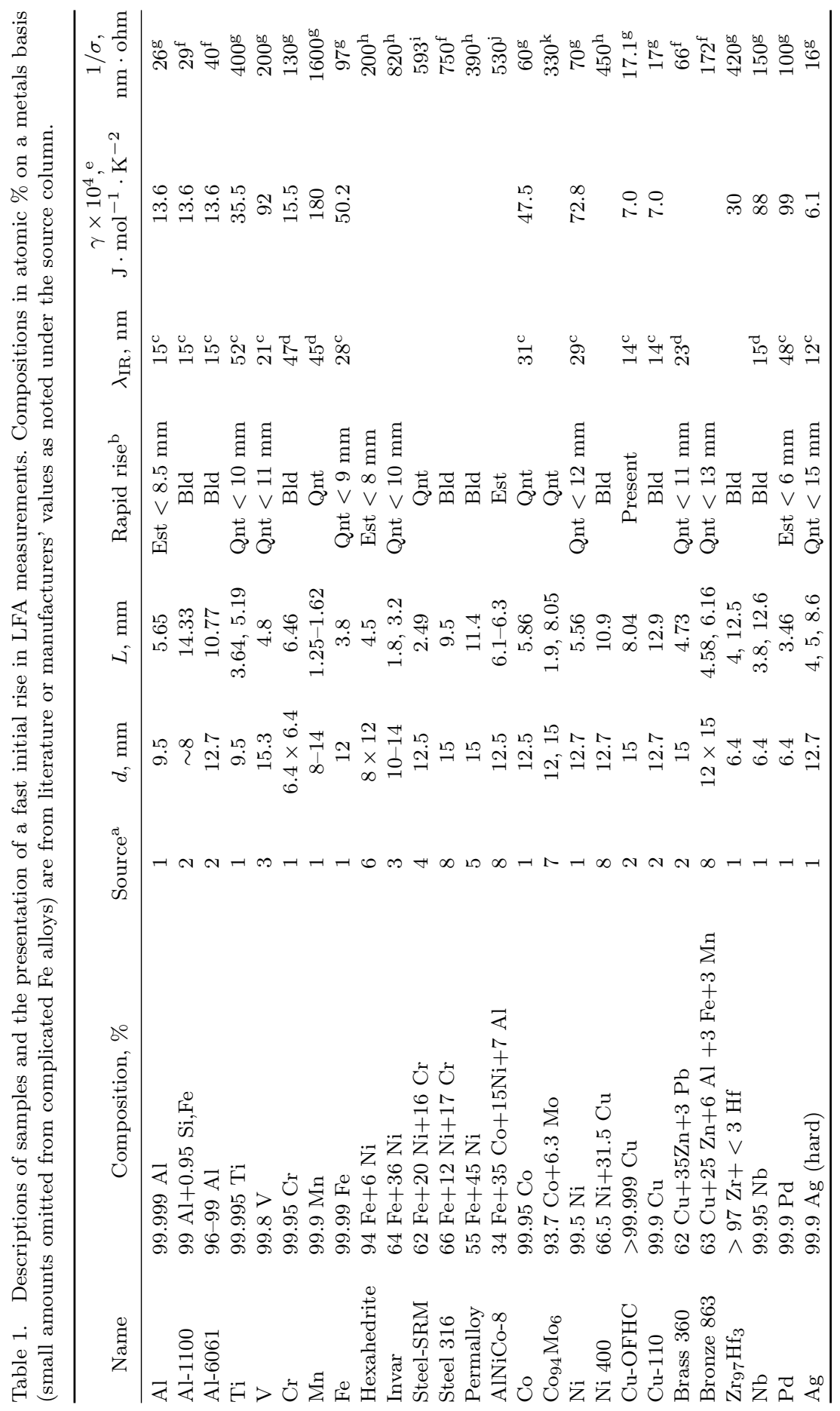




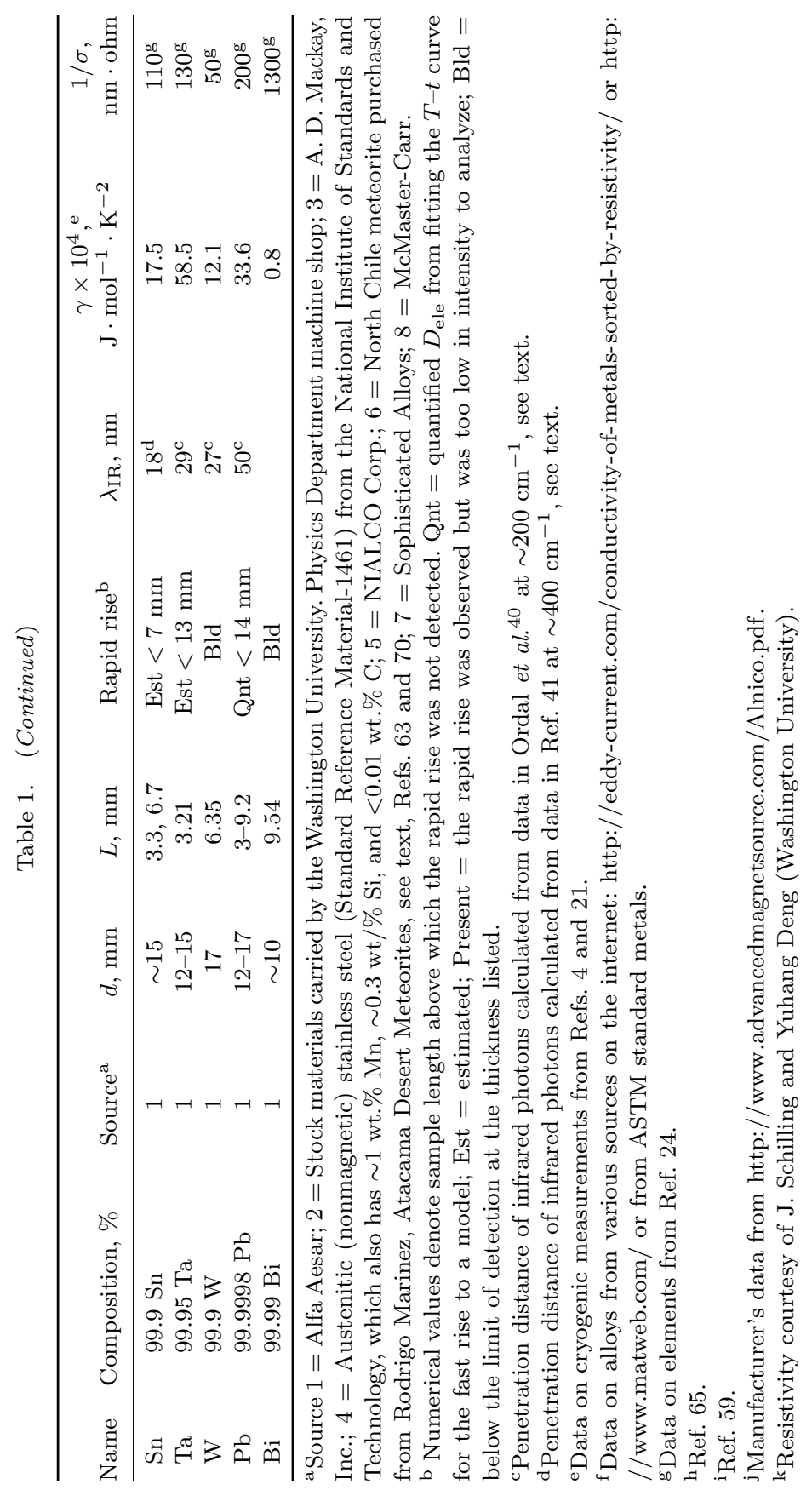


For $\sim 10 \mathrm{~mm}$ lengths, $D$ up to $1000 \mathrm{~mm}^{2} \cdot \mathrm{s}^{-1}$ can be accurately $( \pm 2 \%)$ measured without instrument modification, presuming that the $T-t$ curves are well described by an appropriate model (discussed below). This limit on $D$ (for $L=10 \mathrm{~mm}$ ) is set by the shortest collection duration allowed by our particular software (50 ms), and requires that longer samples be used (Table 1) to determine higher $D$ with this same level of accuracy. We began by probing $>10 \mathrm{~mm}$ lengths, but discovered that $\sim 5 \mathrm{~mm}$ lengths are needed to observe the rapid rise. As discussed below, use of atypically long lengths to probe fast signals degrades the accuracy from nominal (2\%) values generally associated with LFA by nearly an order of magnitude, but is necessary for detection.

\subsection{General procedures for thermal diffusivity measurements}

Operating principles are covered in Sec. 2.2. The main components of our LFA 427 (Netzsch Gerätebau, Germany) are an evacuated furnace with a graphite heating element and water cooled housing, an IR laser, and an InSb detector. For details, see Ref. 66 .

Specimens are mounted by their lower edge using graphite holders in an Ar atmosphere in the furnace (Fig. 5). The temperature dependence of $D$ is obtained by varying furnace temperature, which is measured to $\sim 1^{\circ} \mathrm{C}$ using a type-D W-Re thermocouple placed near the sample, which was calibrated against the Curie transition points of $\mathrm{Fe}$ at $1043 \mathrm{~K}$, and of $\mathrm{Ni}$ at $627 \mathrm{~K}$ for low- $T$ runs. A pulse with $0.8 \mathrm{~ms}$ width from a $400 \mathrm{~W}$ Nd:GGG laser heats the metal sample remotely from below, limiting the initial difference to $<4^{\circ} \mathrm{C}$ across the sample, as required for accurate modeling. This pulse width at $400 \mathrm{~V}$ is recommended for studying metals, because high power $Q$ is needed to offset high heat capacity and density. The same high voltage and width were applied in almost all measurements. However, to better resolve the rapid rise, we used smaller widths in some runs, after confirming that pulse duration does not affect $D$ values. In some experiments on $\mathrm{Ag}, 500 \mathrm{~V}$ with either $0.4 \mathrm{~ms}$ or $0.5 \mathrm{~ms}$ was applied.

To reach $D>1000 \mathrm{~mm}^{2} \cdot \mathrm{s}^{-1}$ expected for electron-electron scattering while maintaining accuracy via lengths exceeding $10 \mathrm{~mm}$, we modified the centering cap to recess the sample bottom into the tube [Fig. 5(b)]. That this modification did not affect the outcome was confirmed by measuring a few $\sim 10 \mathrm{~mm}$ samples with and without the recess. Newer Netzsch instruments accommodate $20 \mathrm{~mm}$ samples.

As heat diffuses across the sample, emissions upward are recorded as a function of time with an InSb detector. Graphite coatings on the sample enhance absorption of the laser pulse and sample emissions. The configuration [Fig. 5(b)] prevents laser light from reaching the detector, which was confirmed using the alignment laser prior to starting each run. If leakage should accidentally happen during the run, (e.g., if the sample falls upon melting), light leakage is evident from saturation of the detector by the intense laser pulse and also from the $T-t$ curve being steep and tall while the laser is fining (for example see Refs. 36 and 53). The configuration 
also prevents laser heating of the centering cone from contributing to the $T-t$ curve (Fig. 5). In some older measurements, a " $\Lambda$ " shape designated as "spark noise" is observed, as a result from light leakage or electrical induction. .53 "Spark noise" is avoided here due to equipment design (Fig. 5). $\frac{66}{}$ Section 3.4 describes additional steps taken to verify that our data on the rapid rise are not corrupted by this problem. Electrical induction is minor and contributes noise to the $T-t$ curves but does not significantly affect measured diffusivity, as discussed below.

Thermal diffusivity values at temperature were calibrated against two standard reference materials from the National Institute of Standards and Technology (NIST): electrolytic iron (SRM-8421), which is highly pure Fe, and austenitic stainless steel (SRM-1461). Applying Cowan's 54 model to our measurements produces $D$ within $2 \%$ of values obtained from $298 \mathrm{~K}$ to $1073 \mathrm{~K}$ on these specific materials using another laser flash apparatus as well as reference data provided by NIST, ${ }^{58,59}$ thereby demonstrating that our instrument attains the uncertainty attributed to this technique. Accuracy of our specific instrument is further evidenced in comparisons involving garnets (YAG, YIG, and GGG), perovskite-structured oxides, alkali halides, $\frac{61}{}$ silica glass, $\frac{67}{} \mathrm{MgO}$ and $\mathrm{Al}_{2} \mathrm{O}_{3}, \frac{68}{}$ diamond and semiconductors,,$\frac{33}{}$ recognizing that earlier measurements of $D$ and $k$ on non-metals often contain systematic errors due to contact resistance and ballistic radiative transfer.

Raw data were obtained at intervals of $\sim 50-100^{\circ} \mathrm{C}$, with several acquisitions (laser shots) at each $T$ setpoint, and processed using the manufacturer's software using Cowan's algorithm ${ }^{54}$ to extract $D$ from the time-dependent emissions (Fig. 6). Each $T-t$ curve corresponds to a single shot. Software corrects for baseline drift during the measurement, and zeros the emissions before the shot, which removes background radiation, temperature drift, and stray light. The shape of the pulse, measured with a light sensor below the furnace, was accounted for. ${ }^{56}$ This sensor also indicates when the shot was fired. Existence of a small initial rise at short time is unimportant to the overall fitting of the slow rise (Fig. 6).

\subsection{Special procedures used to explore the rapid rise}

To probe the rapid and weak rise, which was analyzed using Cowan's ${ }^{54}$ model, we collected data at shorter times and with higher gains than the software recommended. This model is commonly applied to metals. $\frac{38,39}{2}$ The shortest collection time allowed is $50 \mathrm{~ms}$. If the slow rise has a perceptible effect before $50 \mathrm{~ms}$ as in $\mathrm{Mn}$ (Fig. 6), then the software requires using the longer collection time associated with measuring the slow rise. In this case, thermal diffusivity can still be extracted from the rapid rise by truncating the interval used for analysis and fitting [Fig. 6(a), inset]. However, due to the entire $T-t$ curve being comprised of 3000 points, resolution is reduced, and uncertainties are greater, depending on the duration, which controls resolution (i.e., step size).

The software also controls the gain and a physical aperture to produce a signal with height below $9 \mathrm{~V}$. The gain is gauged by the detector response to the emissions 
prior to the shot, and thus signals are less amplified as $T$ increases. Usage of multiple (often atypically long) sample lengths, in conjunction with manual overrides, made measurement of the rapid process in many metals possible. However, because the fast signal involves only a small change in the overall emissions, it has an unavoidably low signal-to-noise $(\mathrm{S} / \mathrm{N})$ ratio, which makes it difficult to process with the commercial software, and inherently difficult to observe at very low and very high temperatures. Below $\sim 400 \mathrm{~K}$, the $\mathrm{S} / \mathrm{N}$ ratio is low in any measurement due to detector characteristics. Measurement at high temperature is hampered because background emissions and thus noise increase more strongly with temperature than the height of the rapid rise. Furthermore, we found that the height of the rapid rise depends somewhat on sample length for a given sample composition. Thus, tradeoffs are involved in its observation.

Importantly, "white" noise accompanying closely spaced data points [Fig. 6(a), shot 3] does not strongly affect extraction of $D$, as long as the shape of the rise is consistent with diffusion, e.g., it is fit by Cowan's 54 model. However, noise can have an effect when extracting $D$ of the rapid rise from curves collected over long times. This happens because small segments of $T-t$ curves have data points spaced far apart relative to the rapid rise profile [Fig. $6(\mathrm{~b})$, curve at $678 \mathrm{~K}$ ]. This reduction in resolution adds uncertainty to the results, which is usually $\sim 20-30 \%$, but depends on the time interval over which the rapid rise is observed compared to the duration of the data collection. Despite the larger uncertainty, these results are important, because acquisitions over long durations allow concomitant study of the slow rise and rapid rise. Specifically, relative rise heights provide a ratio of heat carried by each process.

Collection of many data points (5-10 rather than the typical three per temperature setpoint) was used to improve accuracy at low temperatures, where the rapid rise is weakest, the detector is least sensitive, and the signal is most susceptible to noise. This approach also allows us to maintain statistics when discarding individual runs where truncation created inadequate sampling or where the signal was too weak to properly fit. Large samples and cap apertures were used where possible, because the higher absolute emission improves the $\mathrm{S} / \mathrm{N}$ ratio.

For the short durations, the fits to the initial rise are robust. However, in some cases, the interval prior to the pulse was too short for the LFA software to compute an accurate baseline. For consistent analysis of short shots, a horizontal baseline correction was used instead of the software-controlled (sloped) correction. Usage of a horizontal baseline generally caused only slight adjustments to the extracted $D$ because baseline corrections determined by the software are flat and small over the short time of the rapid rise. The accuracy of the $D$ values obtained using a horizontal baseline is evidenced by the consistency of the three separate shots used per temperature setpoint.

The intrinsic nature of the rapid rise is evident in its associated $D$ being independent of input power, pulse width, sample diameter, sample aspect ratio, amount of the sample that was recessed, signal noise (as long as the rise was distinct and well resolved), or duration of data collection (within the greater uncertainties asso- 
ciated with less-than-optimal resolution). Various tests, detailed below, show that our results are not artifacts. That the rapid rise is a physical property is demonstrated by its shape and is confirmed by the systematic dependence of our $D$ values and relative intensity from the rapid rise on temperature, chemical composition, and certain physical properties. Uncertainties are unavoidable due to the weakness of the rapid rise, which is also a consistent attribute.

\subsection{Recognition and avoidance of spark noise}

Laser light should not reach the detector from the geometry and cross-checks during loading. Laser light impingement was not evident in the presented temperaturetime curves. However, spark noise from heating of the graphite cap is possible if the sample shifted during evacuation or purging or if the centering cone had a tiny crack. We eliminated this potential problem from our data as follows.

To identify this effect, spark noise was intentionally introduced by placing the narrow edge of our rectangular hexahedrite on a centering cone with an aperture slightly larger than the sample width, but with a sample cap aperture that was smaller than the sample width. With this configuration, a small portion of the laser pulse strikes the cap overlying the sample. Due to the cap being thin, a diffusion curve is seen at fairly short time, $\sim 10 \mathrm{~ms}$ (Fig. 7). One of these test runs also involved a rise in emissions while the laser was firing: this run had the larger of the two light leaks and a sample cap aperture that was very close to the sample width. This rise during the pulse probably results from radiative transfer across the very thin lid. Even though the lid is opaque to visible light, a small amount of near-IR light is transmitted. Graphite has a high absorption coefficient, $\frac{69}{\text { but is not opaque }}$ in the near-IR at $\sim 1 \mathrm{~mm}$ thicknesses of the thin caps.

For these contaminated test runs, the apparent $D$, calculated for the fast rise, decreases as a power-law with temperature, consistent with lattice conduction of graphite, but unlike our presented results. Because the length was set to the sample thickness rather than to lid thickness, diffusivity calculated from these test runs does not equal $D$ of graphite. We compared $t_{1 / 2}$ from these contaminated test runs to that calculated from Eq. (9) for lid thickness of $1.55 \mathrm{~mm}$ using data from Poco graphite. Agreement is good, confirming that $T-t$ signals in Fig. 7 are from laser heating of the cap.

The presence of a spark noise error is easy to detect for several reasons. The rise times due to cap heating are always around $5 \mathrm{~ms}$ at $473 \mathrm{~K}$, which is many times the rise times of the rapid rises for all but the longest samples, and increase strongly with temperature. This spurious effect has higher intensity (compared to the slow rise) than do the curves presented below. An adiabatic shape for the erroneous $T-t$ curves is secondary evidence. Suspect runs are not included below. The reported runs showed no evidence of spark noise and had $t_{1 / 2}$ unlike that observed or expected for the graphite cap as in Fig. 7. 


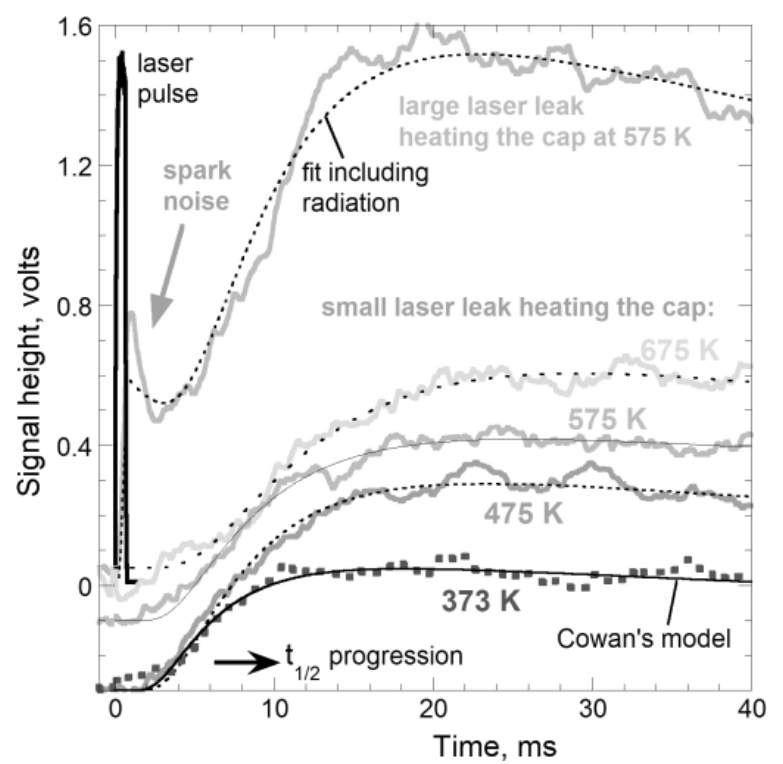

Fig. 7. Characteristic temperature-time curves associated with laser light leakage (spark noise). In these experiments, the rectangular hexahedrite sample purposefully did not cover the aperture of the sample cone, permitting laser light to directly heat the cap. Raw data (gray curves) with fits (black lines) are offset for clarity. Note that voltages are much larger than those in Fig. 6 and most of the other temperature-time curves shown below. The shapes are also consistently adiabatic or nearly so, whereas the rapid rise is followed by radiative cooling to the surroundings, as shown for Mn in Fig. 6.

\section{Results on Diverse Metallic Elements and Alloys}

Over the past decade, our facility has been used to assemble a large database on thermal diffusivity. $\frac{33}{3}$ Our measurements on metals and alloys with thicknesses of 0.5-3 mm (Ref. 70) provide $D$ consistent with previous other measurements using diverse techniques (Refs. 18, 22, 48 and 57-60). For many different metallic elements and alloys, over some range of the temperatures explored (from ambient to melting), we observed a small rise overlapping with or immediately following the laser pulse (Fig. 6). Because such rise characteristics are expected for fast electron transport, and because distinct signals for electrons and phonons should be resolvable (Sec. 2.2), we proceeded to measure many different metals and alloys at varying lengths (Table 1; Sec. 3.1) to understand this phenomenon.

Although both thermal rises are often observed in short samples [e.g., Figs. 6 and $8(\mathrm{a})$ ], quantifying thermal diffusivity associated with the rapid rise generally requires longer samples and shorter collection times than are conventionally applied to metals (Figs. 8-10). Importantly, characterization of the rapid rise, if electronic, is inherently limited by the low carrying capacity of the electrons $\left(C_{\text {ele }}\right)$, their fast speeds, and resistive or magnetic degradation of the electronic heat current (see Secs. 1.3 and 3.3). Because precise characterization of $D_{\text {ele }}$ is not possible, this 


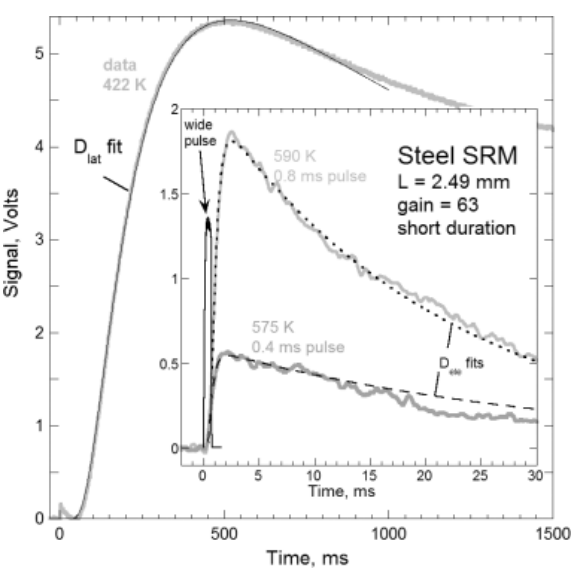

(a)

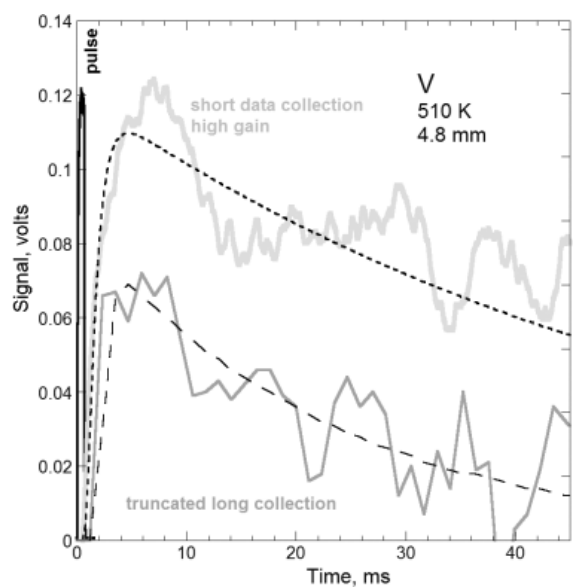

(b)

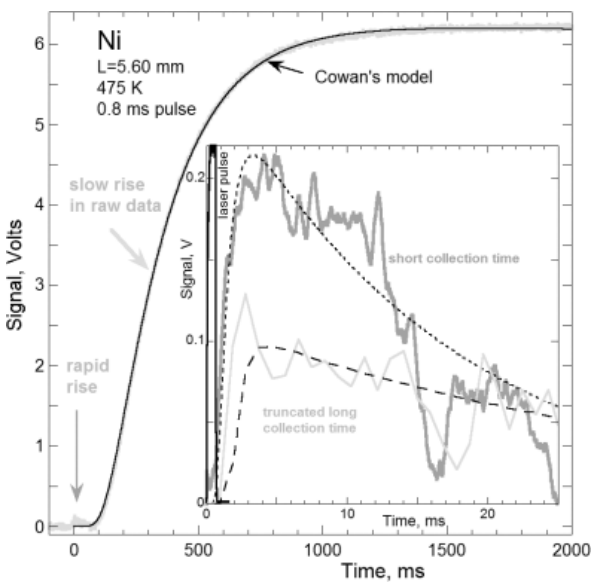

(c)

Fig. 8. Temperature-time curves of transition metals with strong to moderate rapid rises in order of increasing thickness. Gray = baseline corrected data. Black = fits. (a) Nonmagnetic steel, showing collections over long time (main panel) and a short interval (inset). The intensity of the rapid rise correlates directly with the energy input. The large voltage for these two shots is associated with high gain. (b) Element V, showing data collection over long and short times. For the short interval, the slope is determined by the automatic baseline correction which was removed before calculating $D$. Baselines are always correctly computed for the long durations, but the sampling interval creates noise. For short samples (below $5 \mathrm{~mm}$ ), the noise is not a problem for the fits, but does add uncertainty. (c) Nearly-pure Ni. Long samples require a longer collection interval for the main signal (large panel), which causes the truncation for the rapid rise to be noisier (inset).

study instead surveyed transient heating behavior of the electron in diverse metals. We quantified electron behavior in these metals within uncertainties set by the limitations of our equipment, which largely result from the low heat capacity and high speed inherent to the electron. 


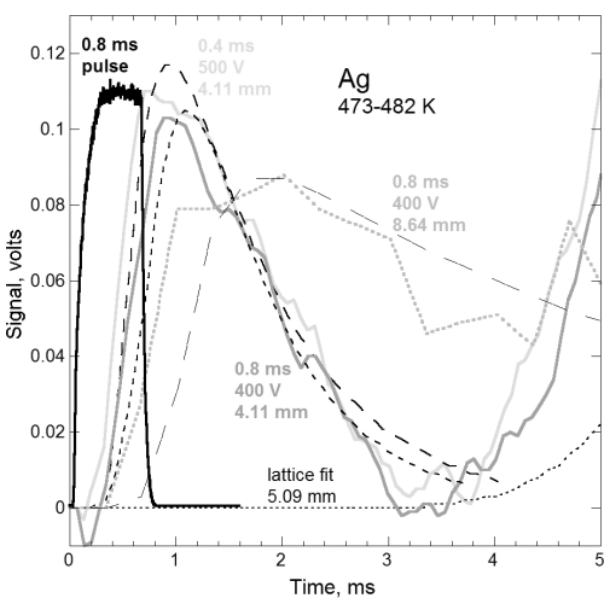

(a)

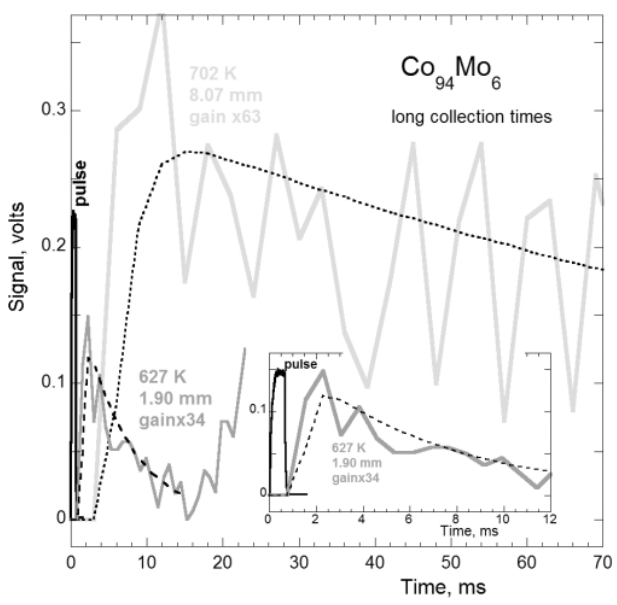

(b)

Fig. 9. Temperature-time curves involving different sample lengths. Gray = baseline corrected data. Black = fits. Heavy black = laser. Doubling the width increases the half-time, but not as much as expected, suggesting $D_{\text {ele }}$ depends on length. As described in the text, electron-phonon scattering removes the hottest electrons and therefore makes the lifetimes increase with length. (a) Silver, showing that the $T-t$ curves are not affected by the width of the pulse, within uncertainty. (b) Slightly impure cobalt showing that temperature affects half-time.

Because the rapid rise is weak (Figs. 6 and 8-10) and would have been masked by spark noise associated with older instruments, $\overline{53}$ such signals were not previously recognized as intrinsic to metals and were not previously studied. Section 4.1 focuses on the raw data. Section 4.2 summarizes why the rapid rise is not an artifact. Section 4.3 describes the intensity of the rapid rise. Section 4.4 examines conditions needed to observe the rapid rise. Section 4.5 discusses assignments of the two rises in the LFA curves. Section 4.6 provides $D$ values for electron-electron scattering and their dependence on $T$. Section 4.7 discusses both the consistency of our observations and the dependence of heat transfer on physical properties. Section 4.8 provides electronic thermal conductivities.

\subsection{Resolution and characteristics of simultaneous rapid and slow rises in temperature-time curves}

Temperature-time curves from long samples of many metals and alloys possess a rapid and weak rise subsequent to the laser pulse. Figures 6 and 8-10 show

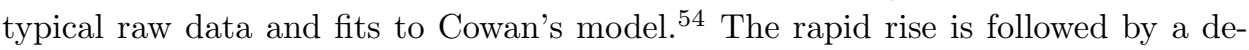
crease in emissions which is terminated by the arrival of slow and strong rise, as shown. This large and slow rise has been explored previously in LFA studies of short samples ${ }^{37,57-59}$ and represents how the majority of heat is conducted through metals. Both short and long samples have dual signals resolvable from the laser pulse [Figs. 6, 8(a), 8(c), and 10(b)]. 


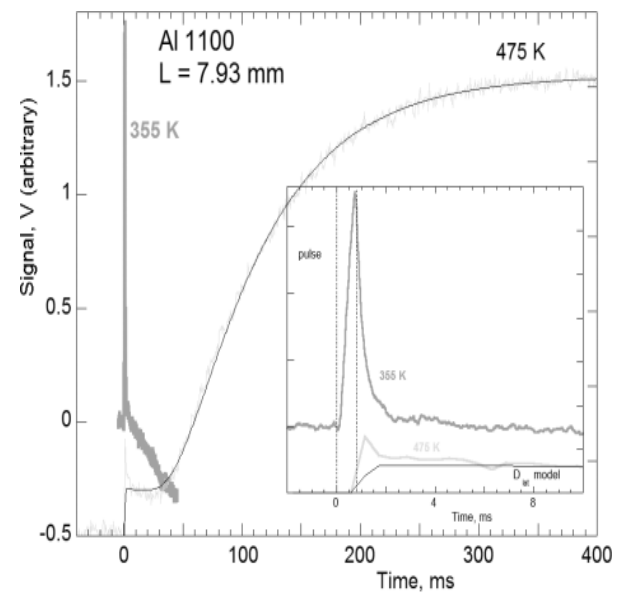

(a)

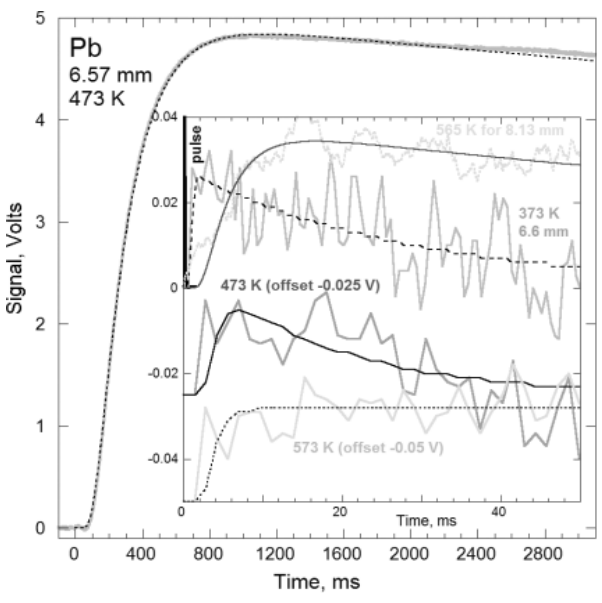

(b)

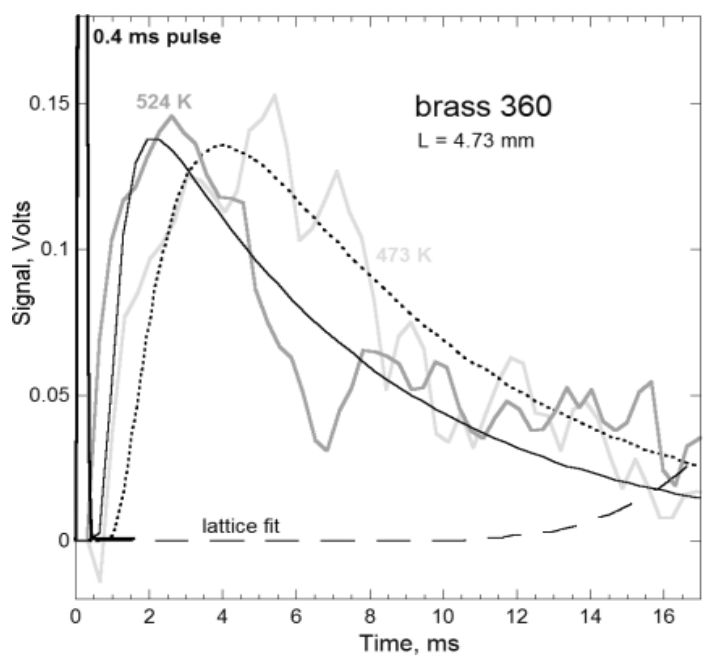

(c)

Fig. 10. Temperature-time curves for nontransition row metals. Symbols as in Fig. 9. (a) Pure aluminum, showing half-time decreases as $T$ increases. (b) Highly pure lead, showing a very weak rapid rise for which the half-time increases as $T$ increases. The noise spacing for the rapid rise increases because the data collection interval increases with temperature. (c) Brass. The rapid rise becomes more rapid as temperature increases.

To establish whether the apparatus is correctly analyzing the $T-t$ signals of the rapid rise, we conducted tests and collected data under diverse experimental conditions. In Figs. 6-10, shot numbers refer to different acquisitions at the same temperature. Unless noted otherwise, voltages of the $T-t$ curves are arbitrary due to variable gain and variable aperture diameter, geared to produce a maximum signal height between $1 \mathrm{~V}$ and $10 \mathrm{~V}$. 


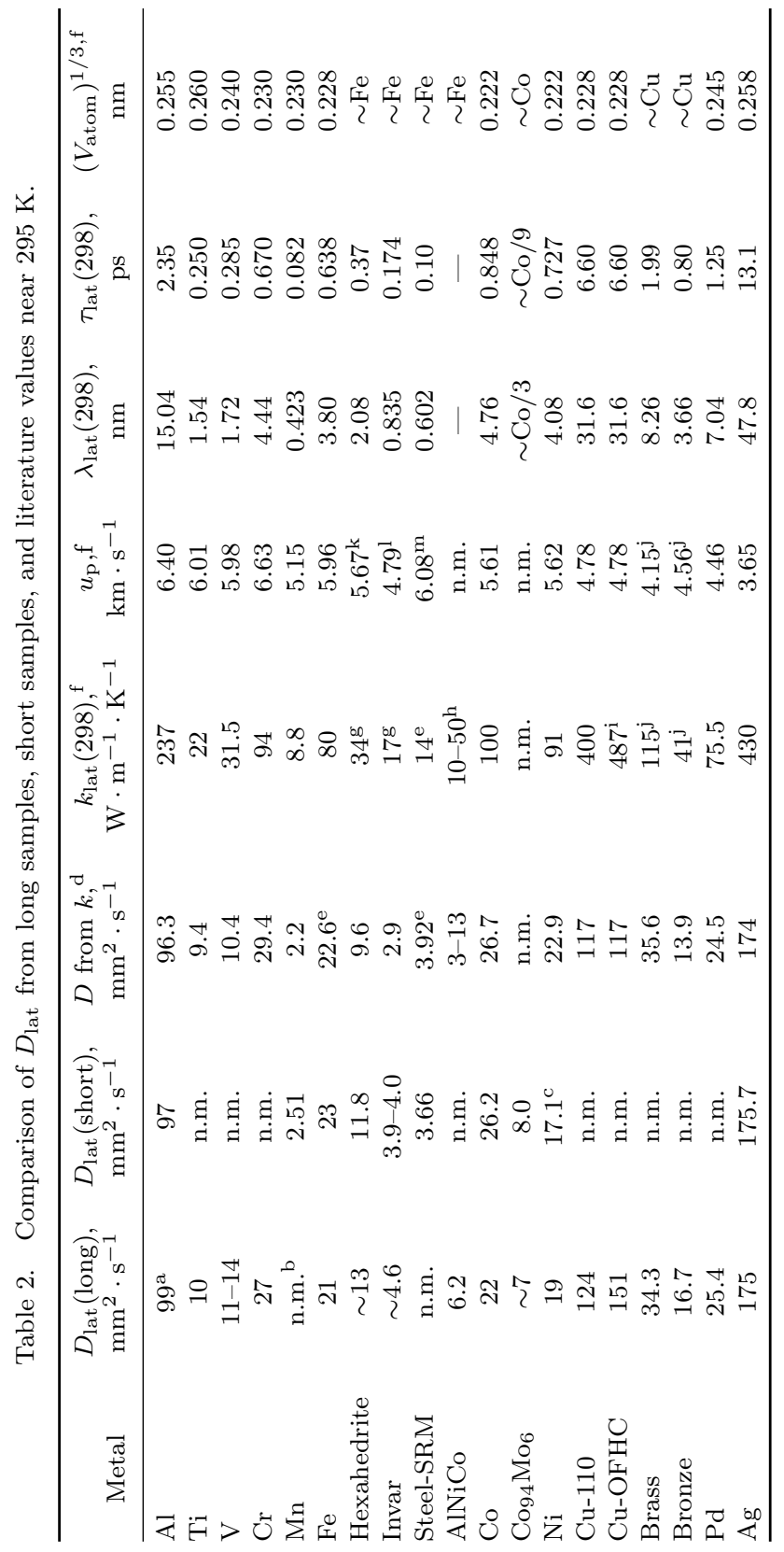




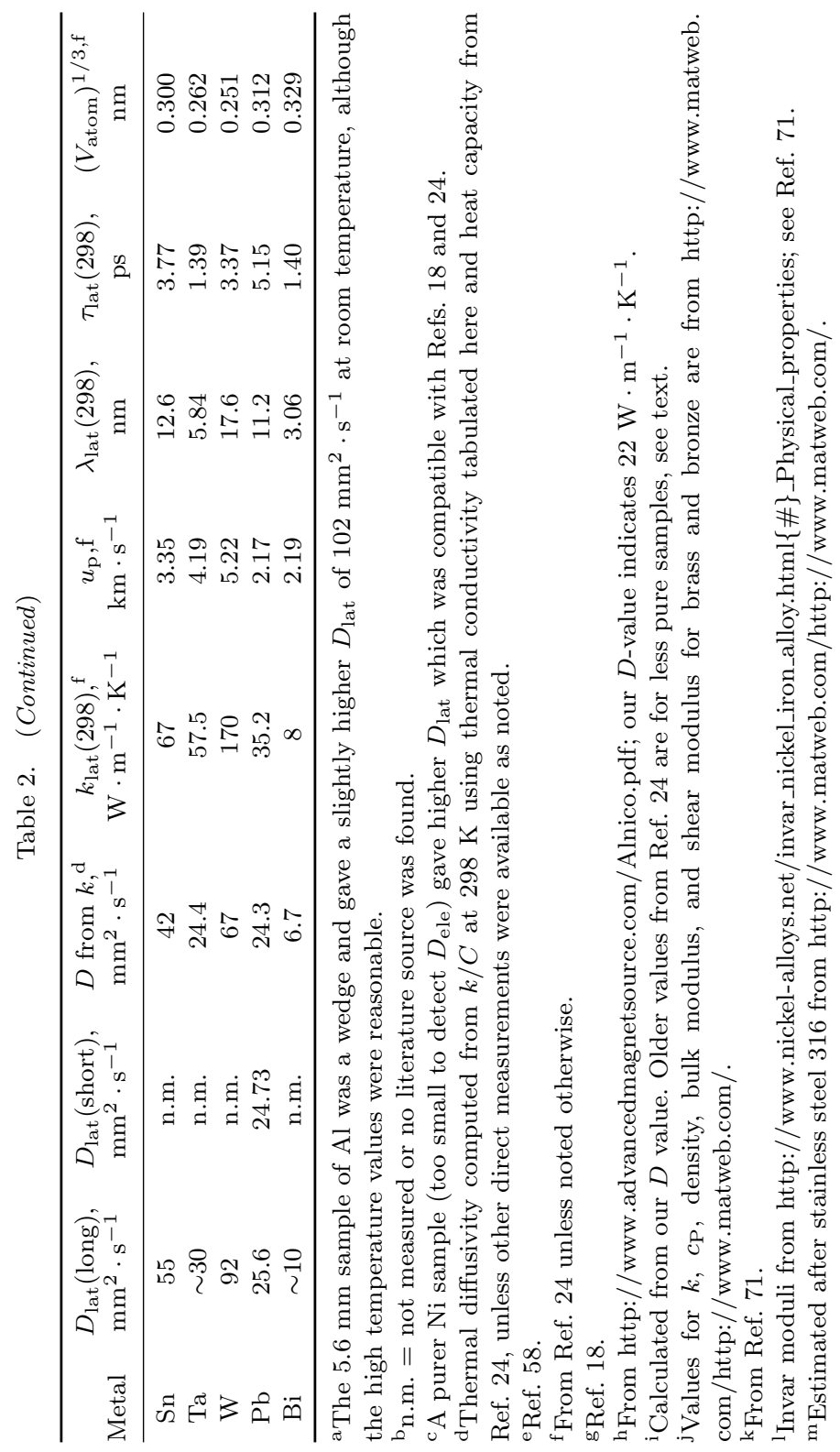


Roughly, $D$ of the slow rise for any given long sample equals that determined from a short section of the same sample or from previous measurements of this composition (Table 2). For the short $(L<3 \mathrm{~mm}$ ) samples, $D$ from the main, slow contribution is optimally constrained. For the long samples, $D$ from the slow rise is less certain than the nominal $(\sim 2 \%)$ error due to long collection times (detector stability limitations) and possible small losses from the sides, because the samples do not follow the ideal of $L \ll d$.

Interestingly, for oxygen-free $\mathrm{Cu}$, our $D_{\text {lat }}$ is higher than values previously measured (Table 2). The $T-t$ curve for $\mathrm{Cu}-\mathrm{OFHC}$ was well matched to Cowan's model ${ }^{54}$ and collection times were short. Previous data on oxygen-free copper predate 1970 (Ref. 22) and purification techniques have improved since then. The older data on $D$ and $k$ (Table 2) compare closely with our measurements of $D_{\text {lat }}$ for $\mathrm{Cu}-110$. Hence, the present result of $D_{\text {lat }}=151 \mathrm{~mm}^{2} \cdot \mathrm{s}^{-1}$ best represents nearly-pure $\mathrm{Cu}$.

Analysis of the rapid rise is more uncertain than of the slow rise because the software collects a set number of points (3000) over the duration of each experiment. Resolution of the rapid rise thus decreases as collection durations increase. Lower resolution in small segments taken from the full $T-t$ curve causes noise to occur at widely spaced intervals whereas noise in a shorter data collection is closely spaced [Figs. 6, 8(b), and 8(c)], and so the fit is better and definitive. Also, collecting data only over the time of rapid rise requires a minimum intensity and that the slow rise begins after at least $50 \mathrm{~ms}$, which infrequently occur together. In addition, for short collection intervals, the baseline is sometimes incorrectly calculated which affects $D$ : in Figs. 8(a) and 8(b), the baseline corrections appear to be correct, whereas in Figs. 6(b) and 8(c), the baseline is overcorrected. Undercorrected baselines should provide a reasonable value for $D$, because the shape is still reasonable (flat, as in adiabatic conditions). We recalculated these short shots using a horizontal baseline and found that the resulting $D$ for three shots constituting one setpoint was tightly constrained. This behavior is consistent with baseline drift being negligible over the short duration of the rapid rise. Therefore, for all short shots, we applied a horizontal baseline correction.

The high noise level mostly results because the rapid rise is intrinsically weak for many metals (Figs. 6 and 8-10) and becomes weaker as sample lengths and temperatures increase (see Sec. 4.3). Thus, characterizing the rapid rise under a wide range of conditions necessarily involves some noise and uncertainty. To reduce uncertainties, our low temperature measurements conducted at the end of our study consisted of 5-10 (rather than three) shots at a given setpoint. We also found that uncertainties were further reduced by using large diameter samples. This occurs because larger samples emit more thermal radiation, so gain can be reduced, thereby improving the $\mathrm{S} / \mathrm{N}$ ratio.

Figures $6,8(\mathrm{a}), 8(\mathrm{c})$ and 10 (b) show that both a fast process and a slow process exist during a single data collection, and that shortening the experimental duration improves the resolution of the rapid rise. Furthermore, shortening experimental duration does not significantly alter the characteristics of the rapid rise so long 
as the horizontal baseline has been correctly computed. Moreover, the rapid rise is always weak compared to the slow rise. Hence, the long lengths are probing the same processes as the short lengths, and potential problems such as large heat losses from the sides are secondary or unimportant. Values of $D$ extracted from the rapid rise are presented in Sec. 4.4 .

\subsection{Why the rapid rise must be intrinsic?}

Experimental artifacts, including spurious (ballistic) radiative transfer, can be refuted as the cause of the rapid rise for several reasons:

- The small, rapid rise cannot be photonic, since temperature does not provide the strong, power-law increase in signal intensity associated with this effect (cf. $T-t$ curves here to those in Refs. 33 and 61). Rather, rapid rise intensity relative to

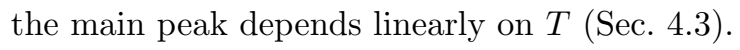

- Furthermore, ballistic radiative transfer should not occur significantly after the pulse, due to the ultrafast speed of light. To check this, we measured a $10 \mathrm{~mm}$ length of transparent, silica glass and found a photonic rise during the laser pulse (not shown).

- Metals having near mm thicknesses are opaque, so the concern is not that light crosses the sample, but rather that the cap is radiatively transmitting. However, the geometry used (Fig. 5) prevents the cap from being directly heated by the pulse. The absence of laser leakage was confirmed with the alignment laser during loading, after evacuating and backfilling the chamber with Ar, and again upon unloading. Hence, only emissions from the sample cap and sample reach the detector but cap emissions cannot cause the small rise because these are included in the baseline. The effectiveness of our precautions were confirmed by the resultant $t_{1 / 2}$ values being too small compared to those obtained by purposefully heating the cap with the laser (cf. Fig. 7 to Figs. 6 and 8-10; Sec. 3.4). Also, spark heating of the cap is always associated with adiabatic $T-t$ profiles regardless of test duration. In contrast, the rapid rise is associated with radiative cooling to the surroundings, which is tempered by the arrival of the slow rise.

- Changes in the temperature of the cap or underlying graphite parts do not contribute to the $T-t$ curves in a way relevant to the rapid rise. Poor physical contact between parts, as well as the removal of background emissions via baseline corrections, prevents changes in the cap or graphite temperature from affecting the $T-t$ curves, with the possible exception of very long times. Long times are not germane.

- Electronic induction does not cause the rapid rise. Not only does the rapid signal vary with composition, length, and temperature, but moreover two electronic boards in the detection circuit were replaced in the course of our experiments. Replacing these boards reduced the noise, but did not otherwise affect this phenomenon. Most of the noise is white, but a small sine wave with a period of $\sim 8.4 \mathrm{~ms}$ exists in some measurements, which probably represents a small 
amount of induction. Both this sine wave and the random noise exist for metals and nonmetals, before and after the laser shot. Both forms of noise are smaller than the rapid rise from $\sim 350 \mathrm{~K}$ to $800 \mathrm{~K}$. Below $\sim 350 \mathrm{~K}$, quantification of the rapid rise was not possible (except in $\mathrm{Mn}$ ) because of low detector sensitivity. At moderate temperatures, the fits average over both types of noise. Above $\sim 800 \mathrm{~K}$, our measurements are again limited because the noise increases more with $T$ than does the height of the rapid rise. This increase in noise with temperature is actually small and is seen in our other measurements on electrical insulators, and thus is instrumental in origin.

- The rapid rise is reproducible. For most samples, several experiments were performed; these runs were separated by hours to years.

Hence, existence of a rapid rise in $T-t$ curves of many metals and alloys, which is followed by a decline in emissions, reflects an intrinsic process. The nature of the process is extracted from the observed behavior (an inverse problem).

\subsection{Relative intensity of the rapid rise and its length and temperature dependence}

A consistent and important characteristic of the rapid rise is its low intensity compared to that of the slow rise (Fig. 11). Near $473 \mathrm{~K}$, where the signal is the least noisy, the rapid rise could be detected when it was as low as $\sim 1 \%$ of the slow rise. At lower $T$, the signal-to-noise ratio increased, limiting detection. Inaccurate data are not included in the fits. Data on thin $\mathrm{Co}_{94} \mathrm{Mo}_{6}$ were collected prior to employing extra precautions to avoid spark noise, but this effect is not evident in the $T-t$ curves and should not be present based on sample diameter. The same holds for measurements of a thin invar sample; however, use of lower laser power resulted in a weaker rapid rise, so that only the trend with temperature is used for comparison. Figure 11 is otherwise based on standard pulse conditions applied to metals of $400 \mathrm{~V}$ and $0.8 \mathrm{~ms}$.

The rise height ratio increases roughly linearly with $T$. For a few samples (bronze and thick invar), the rise height ratio decreases more slowly than linearly near the highest temperatures measured. However, these high $T$ data are insufficient to quantify this decrease in slope. Given the experimental uncertainties, linear fits were mostly used. Exceptions were the thin silver, where noise was reduced at low $T$ via many data collections, and $\mathrm{Co}_{94} \mathrm{Mo}_{6}$, where data were collected over a wide range in $T$. The data on $\mathrm{Mn}$ could be fit to a polynomial, but the linear fit is equally good. Fits in Fig. 11 were not constrained to intersect the origin. The resulting slopes vary with chemical composition and it is clear that the slope is higher below $\sim 400 \mathrm{~K}$ than above.

For most samples, the trends point to zero rapid rise height at some low, finite temperature: intercepts are given in Table 3. Intercepts are near ambient 


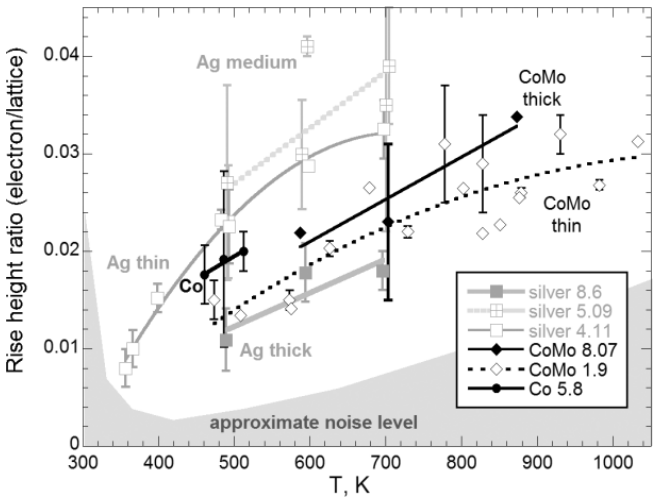

(a)

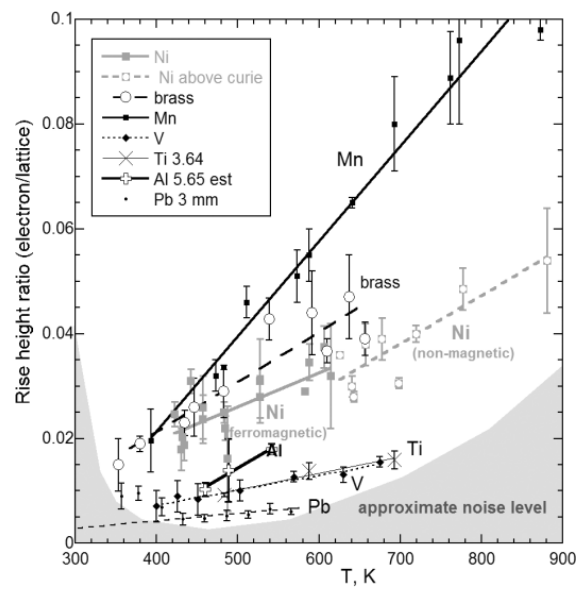

(b)

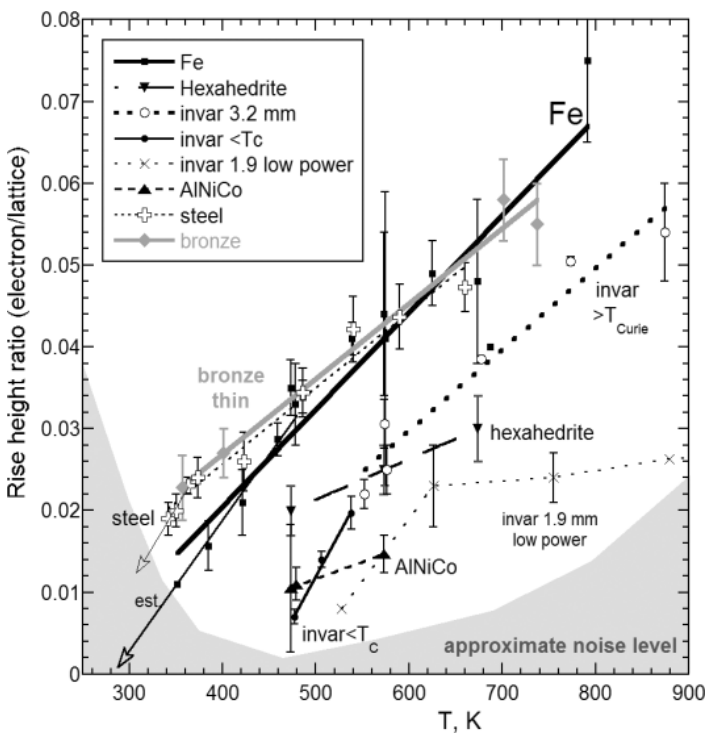

(c)

Fig. 11. Temperature dependence of the ratio of the rapid to the slow rise heights. Fits are linear or quadratic least squares and were not forced through the origin. Samples with few data points or where characteristics are estimated $(\mathrm{Pd}, \mathrm{Sn}$, and $\mathrm{Ta})$ are excluded, but have similar ratios. Noise level depends on many factors: typical values are shown. (a) Comparison of thick and thin samples. For $\mathrm{Co}_{94} \mathrm{Mo}_{6}$, the fits for thick and thin samples are similar, given the uncertainties. (b) Nickel above and below the Curie point and nonmagnetic samples. The trend for $\mathrm{Pb}$ is by inspection where the noise is least, whereas least squares fits are shown for the other samples. Conditions for one run on Mn permitted data collection near room temperature. (c) Magnetic and Fe-rich samples. For invar $(L=3.2 \mathrm{~mm})$, points above and below $T_{\text {Curie }}$ are separately fit. Invar with $L=1.9 \mathrm{~mm}$ is shown only as a rough comparison because these data were collected with lower lase power, which provides a weaker rapid rise. Arrows show the steeper slopes at very low $T$ for steel and Fe. 


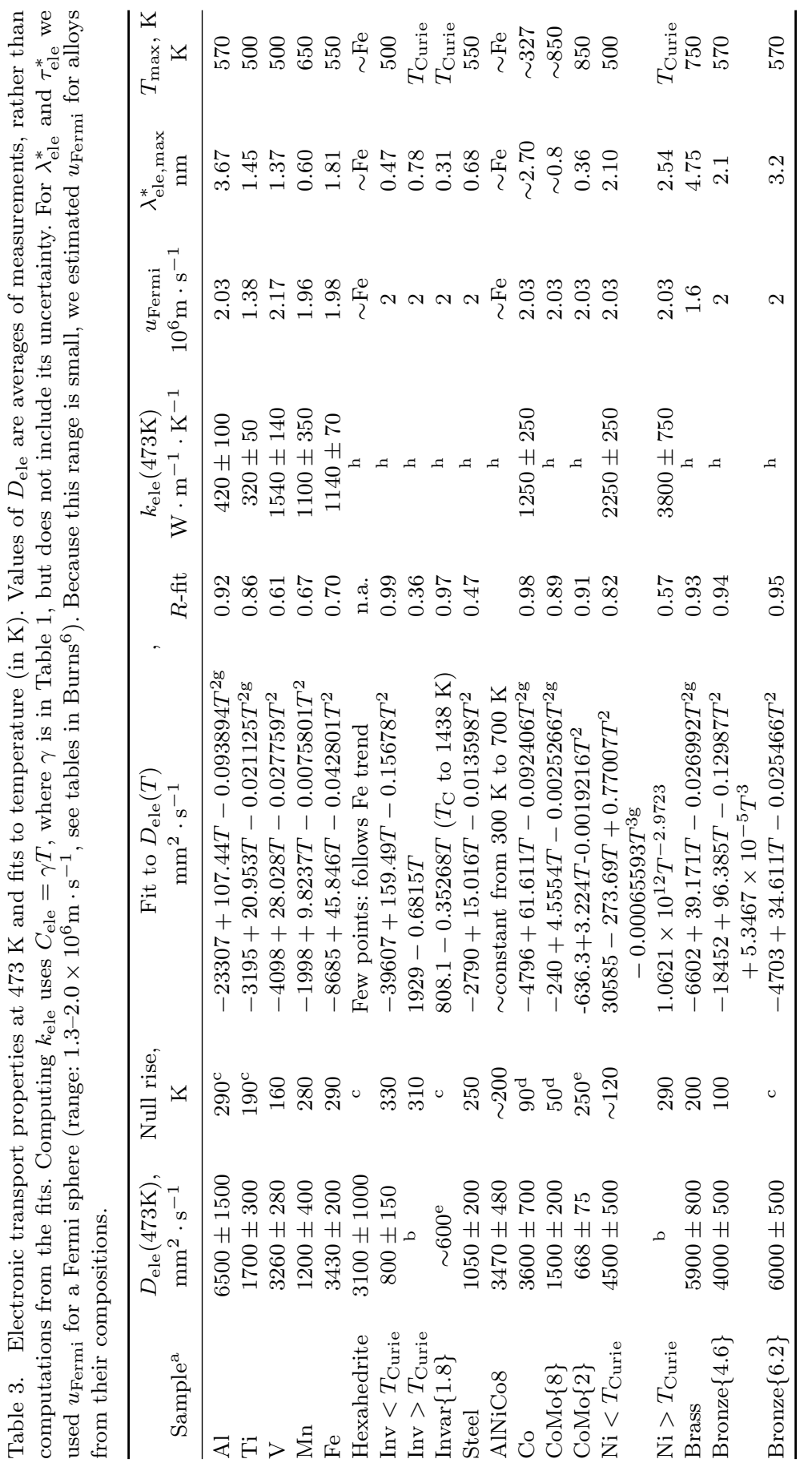




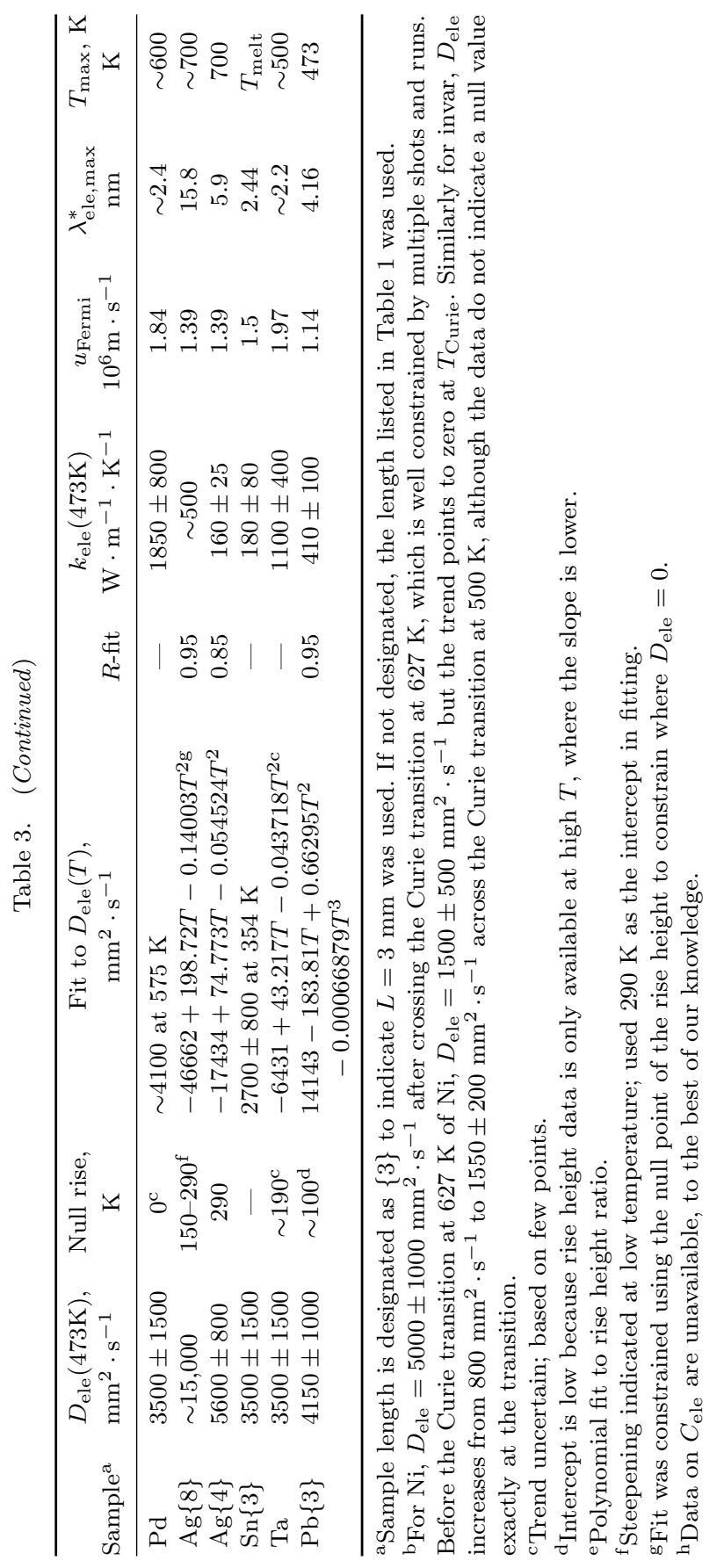


temperature for all materials with well-constrained rise heights below $400 \mathrm{~K}$. The intercept for invar is higher $(440 \mathrm{~K})$ but is reasonably constrained. Intercepts for $\mathrm{Al}$, Ti, thick $\mathrm{Ag}$, and thick $\mathrm{Co}_{94} \mathrm{Mo}_{6}$ are highly uncertain, being based on few data points at relatively high temperatures; calculated intercepts vary from approximately room temperature down to cryogenic temperatures. Data on $\mathrm{Pd}$, $\mathrm{Sn}$, and Ta are insufficient to estimate an intercept. Low temperature intercepts are suggested for $\mathrm{V}$ and $\mathrm{Pb}$ [Fig. 11(b)]. However, below $400 \mathrm{~K}$, the signals are close to the noise level. The well-constrained intercept temperatures from rise height ratios (Table 3) agree with temperatures where the $D$ values extracted from the rapid rise project to zero (Sec. 4.6). All well-constrained intercept temperatures are above $250 \mathrm{~K}$.

The Curie transition seems not to significantly affect the ratio. Within uncertainty, one trend exists for $\mathrm{Ni}$ at all temperatures [Fig. 11(b)]. For invar, data collected below $T_{\text {Curie }}$ follow a steeper trend, but the rise height ratio changes continuously across the transition [Fig. 11(c)]. Ferromagnetism does not appear to control rise height ratio.

Intensity depends on length in a complicated fashion [Fig. 11(a)]. Importantly, the rapid signal is not resolved above the noise for long samples, above $\sim 8 \mathrm{~mm}$ (Table 1). This cutoff length is a rough estimate, and clearly depends on composition. We did not pursue a detailed study of the length dependence: once we realized that $\sim 10 \mathrm{~mm}$ lengths did not resolve the rapid rise, we explored $\sim 5 \mathrm{~mm}$ lengths, which mostly did have a rapid rise.

A minimum length exists for resolution of the rapid rise, which depends on pulse duration $(\sim 0.8 \mathrm{~ms}$ here). With too short a sample $(\sim 1 \mathrm{~mm}$ lengths) the rapid rise completely overlaps the pulse. Although finite pulse width is accounted for in extracting $D$ from the $T-t$ curve, complete overlap may not produce reasonable values of $D$ or peak height, so we do not analyze the rapid rise unless the overlap affects $<1 / 2$ of the rapid rise. With these findings in mind, the data [Fig. 11(a)] indicate that the rise height ratio first increases with sample length, to a maximum when length is $\sim 3-6 \mathrm{~mm}$, and then decreases as length increases. This behavior is observed for $\mathrm{Ag}$, which is the only sample for which rise height was quantified for multiple thicknesses, unlike other materials which were only measured for two different lengths. Data on $\mathrm{Ti}(L=3.6 \mathrm{~mm}$ and $5.1 \mathrm{~mm})$ are not shown, because the rise height ratio is the same within uncertainty. For the very low intensity signal of $\mathrm{Pb}$, the rise height ratio is too uncertain for meaningful comparison of data for different thicknesses. This attenuation is consistent with electron-electron scattering and is attributable to the irreversible loss of electronic heat to the lattice (Sec. 1.3), discussed further below.

\subsection{Conditions under which the rapid rise is observed}

An obvious condition for detection is that the signal must be resolved above the noise (Figs. 6 and 8-10). Holding input power constant in virtually all experiments 
makes detection a meaningful quantity and permits comparison of different materials. The following factors pertain:

- Observation is commonly associated with relatively high $C_{\text {ele }}$ [gray area in Fig. 12(a)]. Electronic heat capacity is calculated from $C_{\text {ele }}=\gamma T$, where Sommerfeld's constant $(\gamma)$ was obtained from cryogenic measurements ${ }^{21}$ and is listed in Table 1.

- The few elements where the rapid rise is detected at low $C_{\text {ele }}$ involve very low electrical resistivity (Al, Ag: Table 1). Long mean free paths are consistent with nearly-free electron behavior, which is further indicated by modeled and measured Fermi surfaces for Al and the noble metals (e.g., Ref. 7).

- High infrared penetration (computed next) also appears germane (Fig. 12). Kramers-Kronig analysis of IR reflectivity data ${ }^{72}$ provides the complex indices of refraction:

$$
n=n_{1}+i n_{2} .
$$

The absorption coefficient $(\alpha)$ is ${ }^{73}$

$$
\alpha=4 \pi \nu n_{2}(\nu)
$$

where $\nu$ is the frequency. Light is attenuated, with penetration depths of $\alpha^{-1}$ (e.g., Ref. 74) which also is the photon mean free path. Many measurements of reflectivity extend down to $200 \mathrm{~cm}^{-1}$ which is appropriate for the black body curve near room temperature. $\stackrel{40}{ }$ Measurements of $\mathrm{Co}, \mathrm{Pd}$, and $\mathrm{Mn}$ begin at $500 \mathrm{~cm}^{-1}$ whereas those of $\mathrm{V}, \mathrm{Nb}, \mathrm{Ru}, \mathrm{Pt}$, and $\mathrm{Sn}$ begin at $800-900 \mathrm{~cm}^{-1} . \underline{41}$ For Fe, the penetration depth decreases from $29 \mathrm{~nm}$ to $26 \mathrm{~nm}$ over this frequency range, which is not a large change. Photon penetration for $\mathrm{Co}, \mathrm{Pd}, \mathrm{Mn}, \mathrm{V}, \mathrm{Nb}$, $\mathrm{Ru}, \mathrm{Pt}$, and $\mathrm{Sn}$ in Table 1 is thus slightly underestimated. Photon penetration depths are somewhat uncertain because tradeoffs exist in determining optical constants from Kramers-Kronig analysis (due to extrapolations to very high and low frequencies ${ }^{75}$ ) and because absolute reflectivity is difficult to measure. ${ }^{41}$

Despite some uncertainties, observation of the rapid rise is associated with large photon penetration depths when $C_{\text {ele }}$ is relatively low; conversely, observation of the rapid rise also occurs in materials with low penetration depths but high $C_{\text {ele }}$ [Fig. 12(a)]. These two factors linearly compensate [Fig. 12(b)]. Thus, observation of the rapid rise is connected with how much heat the electrons can uptake which is governed by both the Fermi statistics of the particular metal and by how deeply the incoming heat reaches into the interior, which excites more electrons. Regarding alloys, this assessment could not be made due to a lack of data on their $C_{\text {ele }}$, and IR reflectivity being rarely measured.

The relevance of photon penetration to heat transfer is consistent with the rise height ratio being positively correlated to laser power. Higher power means a higher number of photons in a given depth, which should stimulate more electrons. 


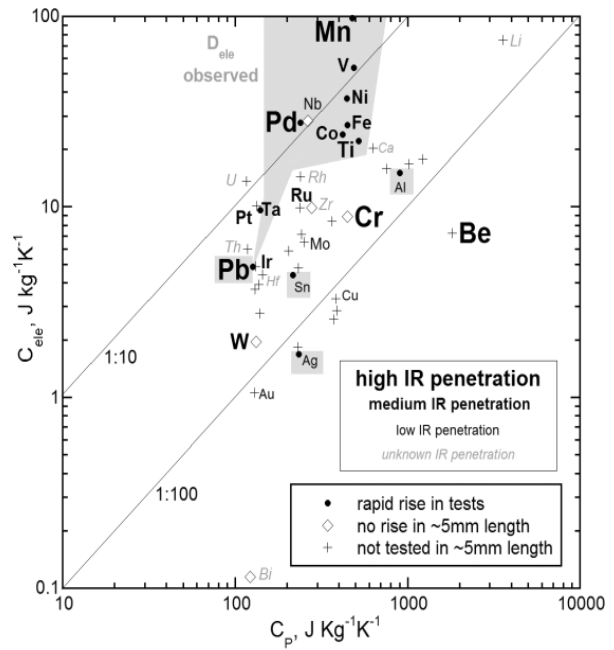

(a)

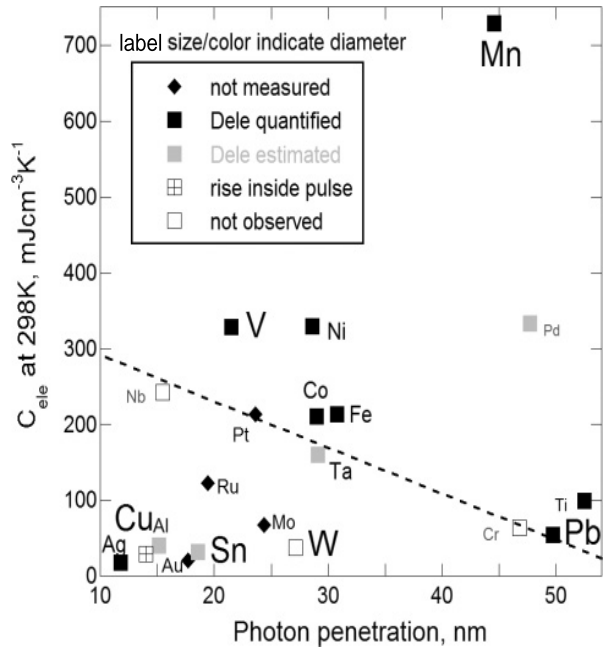

(b)

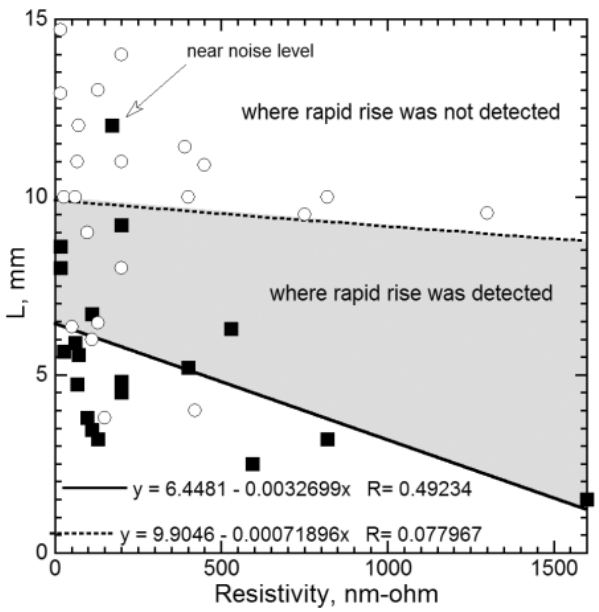

(c)

Fig. 12. Conditions where the rapid rise is observed. Electronic heat capacity from the data in Gopal. ${ }^{21}$ Diameter pertains, due to noise level. Photon penetration depths were calculated from data in Refs. 40 and 41, see text and Table 1. (a) Correlation with lattice heat capacity, where

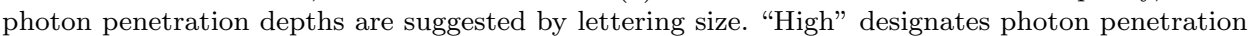
$>40 \mathrm{~nm}$. "Medium" designates 20-35 nm. "Low" designates <20 nm. (b) Map of rapid rise characteristics associated with $C_{\text {ele }}$ and photon penetration. Inset lists the symbols. Large font size for labels designates diameters $>13 \mathrm{~mm}$. Medium font size designates typical $d=12-13 \mathrm{~mm}$. Small font size $=d \sim 9.5 \mathrm{~mm}$. Gray and small font size $=d \sim 6.5 \mathrm{~mm}$. The dotted line divides metals with rapid signals that could be quantified from metals with weak or nonexistent rises. Diameters below $\sim 6.5 \mathrm{~mm}$ inhibit detection. (c) Relationship of the rapid rise with electrical resistivity and sample length. Circles = long samples, indicating the shortest length tested where the rapid rise was no longer observed. Fit and squares = the smallest length tested where the rapid rise exceeded the noise. Gray area shows the range of observation. 
- Sample diameter somewhat affects detection because the $\mathrm{S} / \mathrm{N}$ ratio improves with $d$. The trend in Fig. 12(b) represents a diameter of $12 \mathrm{~mm}$, which describes most samples. Much smaller diameter for $\mathrm{Cr}$ than $\mathrm{Pb}$ explains detection in $\mathrm{Pb}$ only, for which the noise was about a factor of $\sim 4$ lower. Small diameters for Ta and Pd explain why their signals were resolved only near $473 \mathrm{~K}$, where noise is lowest. For large diameter $\mathrm{Cu}$, the rise was observed but entirely overlapped by the laser pulse. For large diameter Sn, the rapid rise was mostly low and contained within the pulse, resulting in useful data acquisitions at only two temperatures.

- Observation of the rapid rise occurs in many classes of metals and alloys. Different electronic configurations, crystal structures, and amounts of disorder are involved. Of the transition elements, the rapid rise is seen in those that are ferromagnetic (Fe, Co, and $\mathrm{Ni}$ at low T), as well as in paramagnetic ( Ti, V, Mn, and Ni at high $T)$. The rapid rise is observed in many different types of metals: simple nonferrous (Al), noble (Ag), transition (listed above, including ferrous), base (Ni), precious $(\mathrm{Pd})$, utilitarian $(\mathrm{Sn})$, and heavy $(\mathrm{Ta}, \mathrm{Pb})$. The rapid rise is observed in slightly impure alloys (hexahedrite, $\mathrm{Co}_{94} \mathrm{Mo}_{6}$ ), in extensive binary cation substitutions (invar), and in alloys with multiple cations (brass, bronze, AlNiCo8, and steel). Of the alloys, magnetism varies from nonexistent (brass, steel, and invar above their Curie points) to strong (AlNiCo8).

- The rapid rise cannot be observed above some moderate length of 6-12 mm, which value depends on composition (Table 1). Although we did not conduct a detailed study of the length dependence of the rapid rise, it is clear that a maximum length exists where the signal can be resolved above the noise and that this length depends roughly on resistivity [Fig. 12(c)].

Hence, observation of the rapid rise given the noise level inherent to our equipment depends on physical properties associated with electrons in metals and alloys (i.e., heat capacity and bulk resistivity) as well as the penetration depth of the graphite's thermal emissions into the sample. Observation of the rapid rise also depends on its half-time since the laser pulse has a finite width. Consequently, our results mostly concern metals and alloys with low to moderate thermal diffusivity values associated with the rapid rise.

\subsection{Why the rapid rise must be electronic whereas the slow rise must be phononic}

An internal origin for the rapid rise is demonstrated by its characteristics and their dependence on certain experimental variables, but not others.

- The $T-t$ shape matching Cowan's model (Figs. 6 and $8-10$ ) proves the process is diffusional.

- Repeatability is demonstrated by shot being similar at each setpoint [Fig. 6(a), 8(a) and 8(b)]. 
- That the same phenomenon is being studied in all experiments is indicated by rise characteristics being roughly similar for diverse metals and alloys at roughly similar lengths and similar temperatures, but not exactly alike (Figs. 6 and 8-11). Thus, a compositional difference exists.

- Halving the pulse width provides half the energy and results in both the rapid and slow rises being about half as intense [Fig. 8(a), same gain for both shots], which is consistent with Eq. (13). Changing the voltage has a similar effect. However, the rapid rise is more dependent on power than the slow rise, which partially results from its proximity to the noise level, but also may stem from the efficiency with which electrons uptake a small part of the incoming heat. Half-times are not affected by these run conditions, nor are they affected by the diameter of the sample, except that increased noise causes greater uncertainty.

- The raw data show that half-time depends on temperature. For $\mathrm{Al}$ and brass, $t_{1 / 2}$ decreases as $T$ increases (Fig. 10), but is roughly constant for others [Fig. 9(b)], which corroborates a compositional dependence.

- The raw data show that $t_{1 / 2}$ of the rapid rise depends on length in a manner that is more complicated than the behavior of the slow rise, which is expected due to differences in electron and phonon behaviors.

- The rapid rise is consistently weak compared to the slow rise, but the relative heights depend on composition, temperature, and, importantly, length (Fig. 11). The length dependence results from attenuation of the electron heat current via interactions with defects and phonons.

- The rapid rise is observed in many but not all metals (Sec. 4.2), indicating a compositional effect, which is controlled by uptake of heat by the electrons, and modified by irreversible loss of heat by the electronic current as it crosses the sample.

Two distinct diffusive rises coexisting in the same $T-t$ curve [Figs. $6,8(\mathrm{a}), 8(\mathrm{c})$ and $10(\mathrm{~b})$ ] requires that two mechanisms independently conduct heat in metals, which is consistent with the known existence of two very different heat carriers. Electrons participating in thermal conduction in metals have fast speeds of $\sim 10^{6}$ $\mathrm{m} \cdot \mathrm{s}^{-1}, \underline{4}$ which should provide rapid (transient) heating. We therefore interpret the small, rapid rise in LFA curves of metals (Figs. 6 and 8-10) as transient heat flow of electrons and the subsequent large, slow rise as phononic. The later assignment is supported by the large, slow rise being similar to $T-t$ curves of simple insulators $\left(\mathrm{MgO}, \mathrm{Al}_{2} \mathrm{O}_{3}\right)$ with comparable thickness (see Ref. 36). Specifically, at $298 \mathrm{~K}$, $D=23 \mathrm{~mm}^{2} \cdot \mathrm{s}^{-1}$ for $\mathrm{Fe}$, which compares reasonably with $15.3 \mathrm{~mm}^{2} \cdot \mathrm{s}^{-1}$ and $12 \mathrm{~mm}^{2} \cdot \mathrm{s}^{-1}$ for $\mathrm{MgO}$ and $\mathrm{Al}_{2} \mathrm{O}_{3}$, respectively 68 : see Fig. 4 for comparisons with elements. The phononic nature of the slow rise in metals is verified in Sec. 6.1 using a preexisting database on $k$. This comparison and the above results show that the slow rise in the $T-t$ curves provides $D$ values which correctly describe both steady-state and transient heat conduction, although these $D$ values cannot correctly describe the immediate response of a metal to a heating pulse. Furthermore, the rapid rise, being small in height and declining rapidly with time, does 
not measurably affect extraction of $D$ from the gradual, but large, rise, but instead governs transient heat conduction over only very brief intervals.

It is self-evident that the rapid rise cannot be phononic, because it is faster than the slow, primary rise. Furthermore, the rapid rise cannot be photonic (Sec. 4.2). The ratio of peak heights that projects to $\sim 1 \%$ near ambient temperature (Fig. 11) is also consistent with our assignment of the weak signal to electrons. From the Pauli exclusion principle, only about $\sim 1 \%$ of the electrons can uptake, and thus transfer, heat, due to low $C_{\text {ele }}$. Phonons being much more efficient at uptake is consistent with heat energy being similar in value to vibrational energy and with measured heat capacity being largely phononic. $\stackrel{4,6-8}{-8}$

The rapid rise must be electronic, as only this carrier type satisfactorily explains our observations. Mechanisms are discussed further below, with reference to our model (Sec. 5). Here we note that observing heat transported across the entire sample by the electrons is predicated on several factors:

- Sufficiently short times and long samples are needed to observe high $D_{\text {ele }}$, which is expected from high Fermi velocities [Eqs. (14) and (15)].

- Electrons near the bottom surface must be excited by infrared emissions of the graphite coating (Fig. 5). Therefore the amount of heat received by the electrons depends on the distance that the infrared photons penetrate (since power was held constant), which is calculated in Sec. 4.4 from IR reflectivity data, and the electronic heat capacity, combined, as shown in Fig. 12.

- The experiments record the rise in temperature at the top surface (Fig. 5) as this heat is lost to the surroundings. Loss to the surroundings is essential: if the electrons release their heat during their journey across the sample, it will be promptly reabsorbed. If another electron receives this energy, the process will continue: in essence, the electronic process consists of energy diffused among the participating electrons. However, this heat energy can only be transferred down the thermal gradient. Any heat transferred to the phonons progresses slowly to the topside and is not recorded in the rapid pulse. The amount of electronic heat lost to the lattice is represented by the resistivity. However, differences between electronic conduction, where resistivity measurements are possible, and thermal conduction by electrons may result in different applicable resistivities because the energy and number of carriers involved differ substantially.

- It is also relevant that the signal is not observed once the length exceeds some value, $\sim 6-10 \mathrm{~mm}$, depending on composition at constant diameter (which affects the $\mathrm{S} / \mathrm{N}$ ratio). This behavior is consistent with some proportion of the excited electrons releasing their heat energy to the lattice, whereupon this energy becomes part of the phononic heat current. At some long length, the electronic heat current is attenuated to a level that is below our detection limit.

- Low resistivity limits the conversion of electronic heat to phononic heat, which explains why the signal is observed in $\mathrm{Al}$ and $\mathrm{Ag}$, despite their low $C_{\text {ele }}$ and low IR penetration. Although fewer electrons uptake heat in these metals, degrada- 
tion of the electronic heat current with distance is greatly reduced, permitting observation.

\subsection{Results on $D_{\text {ele }}$ and its dependence on temperature, length, and transitions}

Figures 13-15 show thermal diffusivity extracted from the rapid rise as a function of temperature for all samples, except $\mathrm{Sn}$ and $\mathrm{Pd}$, for which data are few and uncertain. Table 3 reports $D_{\text {ele }}$ at $473 \mathrm{~K}$, where it could be determined with reasonable accuracy for all samples. This value represents our best determination, computed by averaging over a small range of temperatures, and is independent of the fits.

The $T$ dependence is generally best described by least squares fitting to a secondorder polynomial, listed in Table 3. For Sn and $\mathrm{Pd}$, with only two temperature measurements, $D_{\text {ele }}$ at the second temperature is reported. Table 3 and the figures indicate if these fits were unconstrained or if the temperature, where the rapid rise projects to the null value (the intercept), was utilized in the fitting. The constrained and unconstrained fits differ only at low temperature, below where $D_{\text {ele }}$ could be extracted from the $T-t$ curves. Using the intercept from the rise height ratio as a fitting constraint (elements $\mathrm{Al}, \mathrm{Ti}, \mathrm{Mn}, \mathrm{Co}$, and $\mathrm{Ni}$ ) allowed us to calculate $k_{\text {ele }}$ at $298 \mathrm{~K}$ for comparison to the WF law (Sec. 4.8). This constraint was necessary when the fitting suggested that $D_{\text {ele }}=0$ at temperature where the rapid rise existed but its value cannot be reliably ascertained due to low intensity.

Although $D_{\text {ele }}$ responds differently to $T$ for our samples, many commonalities exist. Key findings are that thermal diffusivity extracted from the rapid rise is large $\left(\sim 1000 \mathrm{~mm}^{2} \cdot \mathrm{s}^{-1}\right.$ for alloys and $\sim 4000 \mathrm{~mm}^{2} \cdot \mathrm{s}^{-1}$ for most elements), and depends on temperature, structural or magnetic phase transitions, and length, sometimes strongly (Figs. 13-15; Table 3). A compositional dependence obviously exists, as discussed in detail in Sec. 4.7. Such variations are sufficiently distinct that trends can be discerned despite large uncertainties. Effects of various experimental parameters are discussed next.

Data on $D_{\text {ele }}$ acquired using short and long collection times generally agree within uncertainty, as shown in our most detailed measurements (Figs. 13 and 14). However, for very fast rises, truncating the collection time can reduce accuracy, and can yield either over- or under-estimates for $D_{\text {ele }}$. This variability results from a combination of high noise and resolution limitations. For the remaining materials, collection durations are not generally distinguished. Sample diameter (tested for $\mathrm{Pb}$ and $\mathrm{Mn}$ ) does not affect $D_{\text {ele }}$ values, although smaller $d$ is associated with greater uncertainty because the $T-t$ curves are noisier. Similarly, reducing laser power or shortening pulse duration also increases the noise and thus the uncertainty of $D_{\text {ele }}$, but does not appear to alter its value.

Length affects $D_{\text {ele }}$ and the rise height ratio in opposite directions (Fig. 16). Our quantitative data on the rapid rise as a function of $L$ are limited to very few samples, although all samples have no measurable rapid rise above some minimum $L$ 
of $\sim 6-14 \mathrm{~mm}$. The available information indicates a roughly linear decrease in rise height with $L$. However, a power-law is more likely from the perspective of the heat current being attenuated with length, via interactions with defects and/or phonons. Electronic thermal diffusivity increases with $L$, apparently following a power-law. Although linear fits to $D_{\text {ele }}(L)$ are permissible, these do not give reasonable values for $D_{\text {ele }}$ for small lengths. The limit of $D_{\text {ele }} \rightarrow 0$ as $L \rightarrow 0$ is consistent with

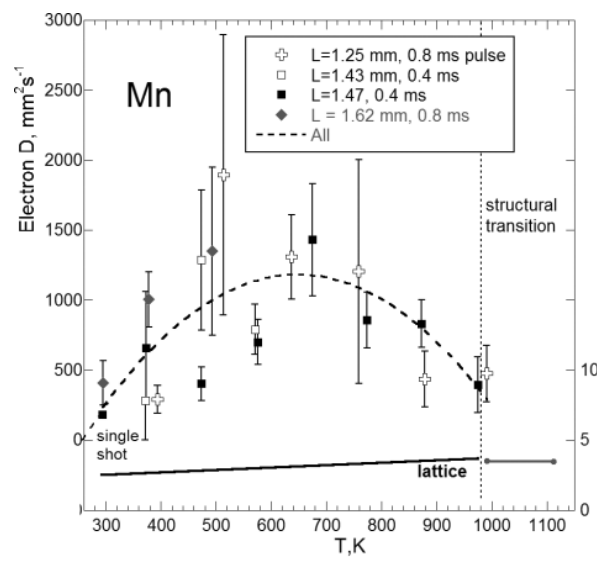

(a)

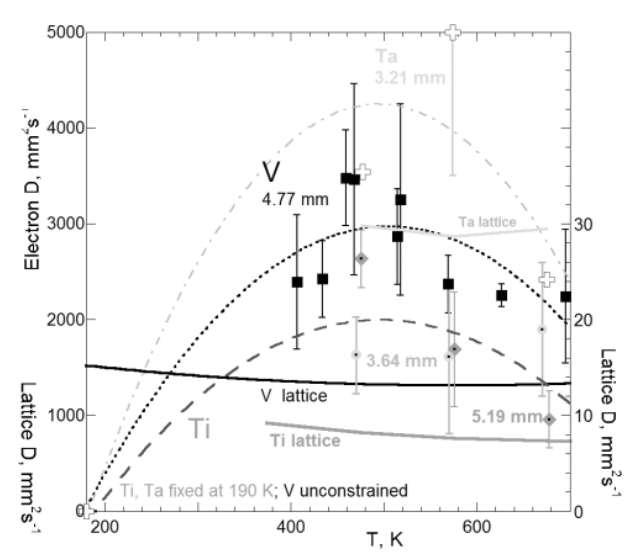

(b)

Fig. 13. Temperature dependence of $D_{\text {ele }}$ for elements. The electronic contribution (left axis) is shown as symbols with error bars and a least squares fit (listed in Table 3). The lattice contribution (right axis) is shown as a heavy line (or lines), generally without symbols, and is labeled. The right and left axes always differ by a factor of 100, but in some panels their zeros are offset for clarity. (a) Data on Mn gathered from four different plates, indicated in the legend. The structural transition affects the slope of the lattice contribution. The effect on the electronic heat transfer is uncertain. Values for $D_{\text {ele }}$ of Mn are low compared to those of other elements. The intercepts for the fit were not constrained. Due to porosity, data for different lengths were not distinguished in the fitting. (b) Data on moderate lengths of $\mathrm{V}$ (black), Ti (medium gray), and Ta (light gray, open symbols). For Ta, data are few and uncertain: error bars are only shown for one point, for clarity. Fits to $D_{\text {ele }}$ of Ti were constrained at $190 \mathrm{~K}$ where the rise height is null: Ta was treated similarly, for consistency. (c) Data on electron transport from the same rod of $\mathrm{Ni}$, which was acquired in many different runs. Filled squares $=$ short data collection times. Open squares $=$ truncated, long collection times. Each point represents data collected during a run. However, at $373 \mathrm{~K}$, two experiments performed on the same day under the same conditions were averaged. Differences in $D_{\text {ele }}$ between short and long collection times above the Curie point are attributed to the very high values not being well constrained for the lower resolution and higher noise at high $T$. The same trends occur for long and short collection times. At the Curie point, while the transition was occurring, the results vary widely $\left(1870-7000 \mathrm{~mm}^{2} \cdot \mathrm{s}^{-1}\right)$ and were not included in the fits. Fits include short and long collection times. Above $T_{\text {Curie }}$, a power-law fit best describes the data. The magnetic transition has a much smaller effect on $D_{\text {lat }}$ (from a thin, $1.6 \mathrm{~mm}$ cut. (d) Data on three pieces of high purity $\mathrm{Pb}$, which were each compressed in a vice to provide large diameter and data on many lengths (listed in the inset). However, the electronic contribution is very uncertain for the long samples due to $\mathrm{Pb}$ having a very weak signal. The lattice contribution is shown in gray; and the melting point in light gray. Premelting effects are seen. Temperatures were calibrated based on melting. 


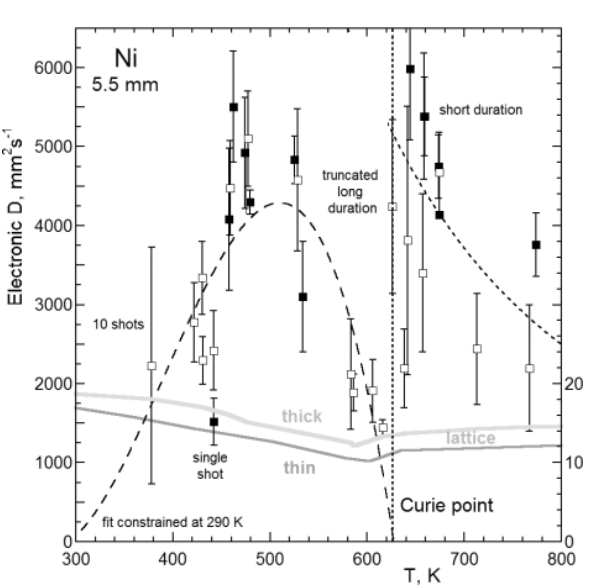

(c)

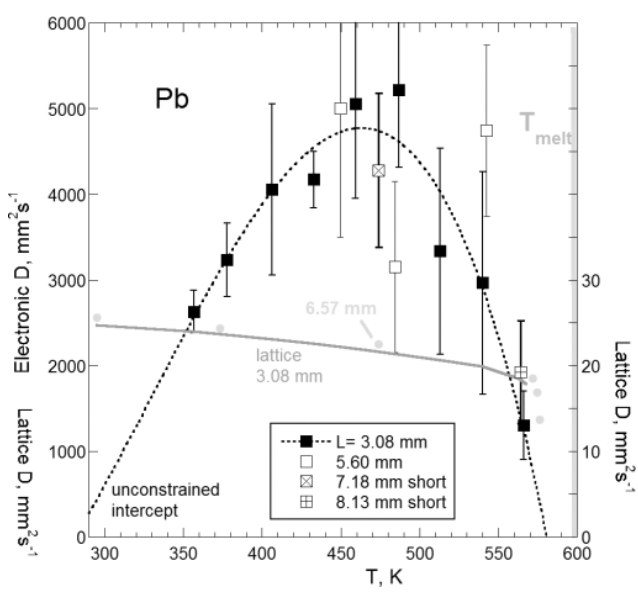

(d)

Fig. 13. (Continued)

electronic transport being a perturbation on the dominant, phononic, heat transport. The increase in $D_{\text {ele }}$ with $L$, while the rise height ratio decreases, is consistent with fewer collisions occurring as the heat current is depopulated, and so the mean free paths increase with $L$ (discussed further below).

The rapid rise could only be resolved at ambient temperature for $\mathrm{Mn}$, which has the largest $C_{\text {ele }}$ by far. By $\sim 350 \mathrm{~K}, D_{\text {ele }}$ could be extracted for many samples, particularly those with large diameter, because this attribute provides greater absolute emissions and improved $\mathrm{S} / \mathrm{N}$ ratio. We also collected more shots at low $T$ to improve statistics. Initially, $D_{\text {ele }}$ increases with $T$, reaches a maximum at a modest temperature (450-900 K, depending on the composition), and then decreases as $T$ further increases. Observing and constraining the maximum mostly depends on the range of temperatures explored. For example, if the measurements do not reach sufficiently high temperature, only the increase in $D_{\text {ele }}$ with $T$ is seen (brass, $\mathrm{Al}$ ) or the trend becomes flat but not obviously decreasing (Ag). In contrast, if the measurements do not reach sufficiently low $T(\mathrm{Co})$, only the decrease in $D_{\text {ele }}$ with increasing $T$ is observed. If the range is small and only at moderate temperatures, a flat trend is seen $(\mathrm{Ti})$. In all samples where data were collected over a fairly large range of temperatures, a maximum in $D_{\text {ele }}$ exists at some moderate $T$. For invar below $T_{\text {Curie }}$, a peak is suggested even though the $T$ range is small; we report the polynomial because the rise height ratio intercept at $440 \mathrm{~K}$ matches the intercept from the fit to $D_{\text {ele }}(T)$.

Magnetic and structural transitions clearly affect the trends in Figs. 13-15: just below any transition, as $T$ increases, $D_{\text {ele }}$ decreases. Upon crossing the Curie point, $D_{\text {ele }}$ abruptly increases, after which $D_{\text {ele }}$ decreases as $T$ increases. Due to the large number of points collected for $\mathrm{Ni}$, its high temperature trend can be constrained with a power-law fit. Other samples also show a flattening in the $D_{\text {ele }}$ trend at 


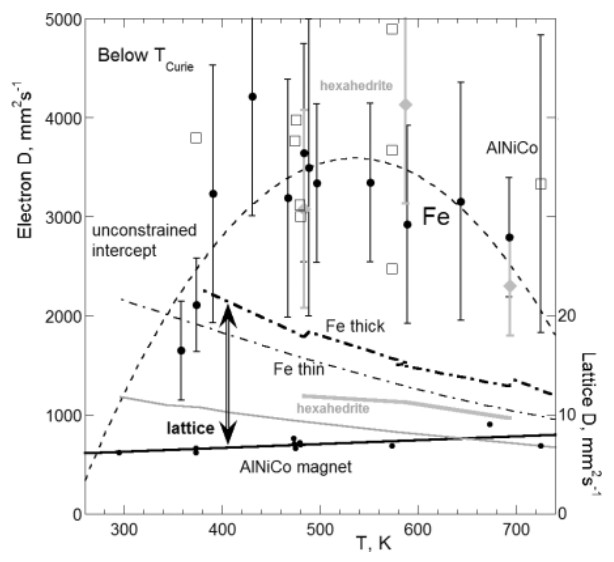

(a)

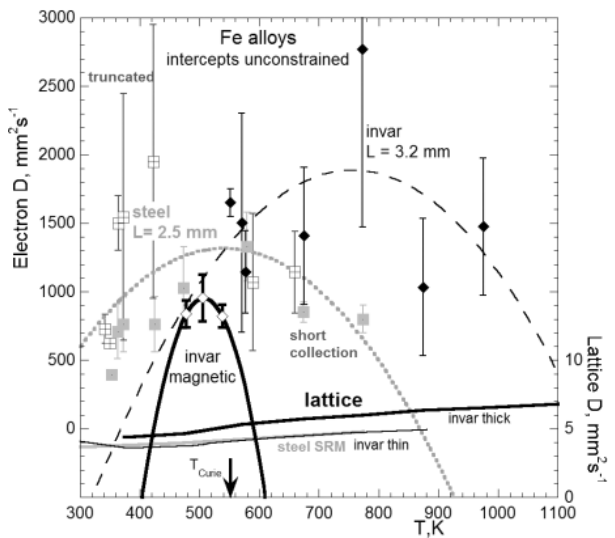

(b)

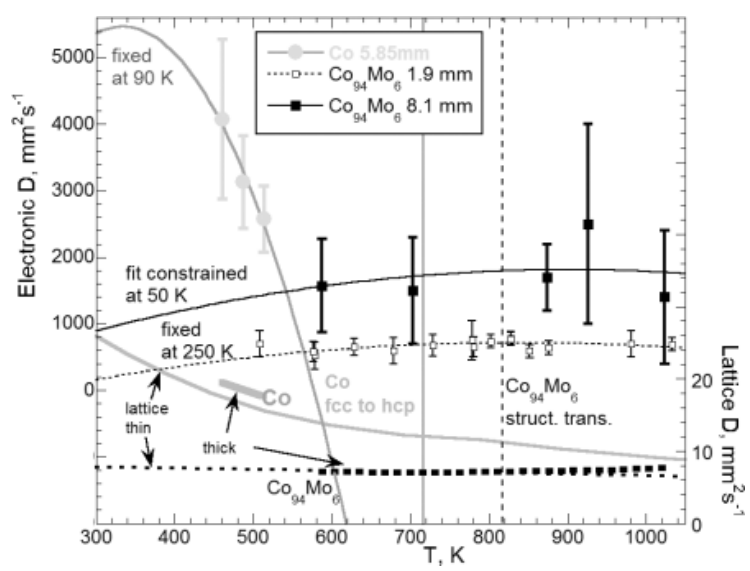

(c)

Fig. 14. Temperature dependence of $D_{\text {ele }}$ for transition row alloys. See Fig. 13 for most symbols. (a) Magnetic alloys of $\mathrm{Fe}$ with predominately $\mathrm{Ni}$ (squares and gray diamonds) compared to elemental Fe (dots), all below the Curie point. For the lattice, results from thin $(<3 \mathrm{~mm})$ and thick $(3-7 \mathrm{~mm})$ samples are shown as thin and thick solid lines at the bottom of the graph. For $D_{\text {ele }}$ of AlNiCo8, a typical error bar is shown: data are mostly single $T-t$ acquisitions from different samples of nearly-identical size with poles oriented both parallel and anti-parallel to heat flow. The point at $3770 \mathrm{~mm}^{2} \cdot \mathrm{s}^{-1}$ at $473 \mathrm{~K}$ is an average of three short shots. (b) Mostly nonmagnetic Fe alloys. Gray $=$ nonmagnetic steel-SRM. Diamonds $=$ invar with $L=3.2 \mathrm{~mm}$, where open diamonds show $D_{\text {ele }}$ below the Curie transition. X $=$ invar with $L=1.9 \mathrm{~mm}$, where the linear fit well describes the data up to $1438 \mathrm{~K}$. Intercepts are not constrained. (c) Magnetic alloys of Co (black) compared to the element Co (gray). Effects of the structural transitions are evident only in the lattice contribution for Co. For $D_{\text {ele }}$ of Co, a constrained polynomial fit is shown: above ambient temperature, this differs little from a linear fit. For the alloy, fits to $D_{\text {ele }}$ are shown, but a constant value also represents the data, which is length-dependent. Given the low amount of cation substitution, the Curie transition should occur above the temperatures explored, as is the case for low Ni substitution in the hexahedrite. 
high temperature, but insufficient points exist to constrain such trends. In contrast to magnetic changes, the structural transitions in $\mathrm{Mn}$ and $\mathrm{Co}_{94} \mathrm{Mo}_{6}$ did not affect $D_{\text {ele }}$ within uncertainty, although the scant data limit exploration of structural

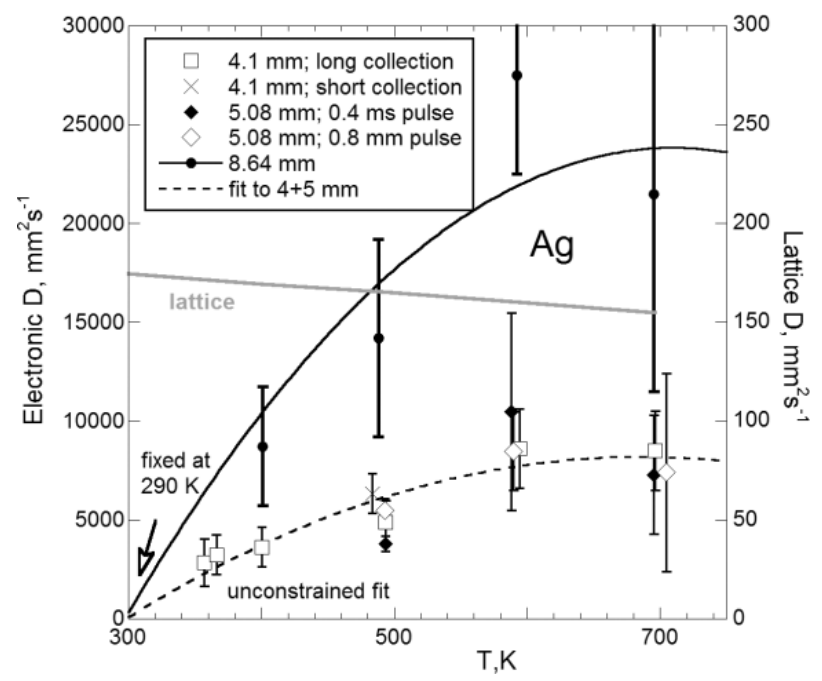

(a)

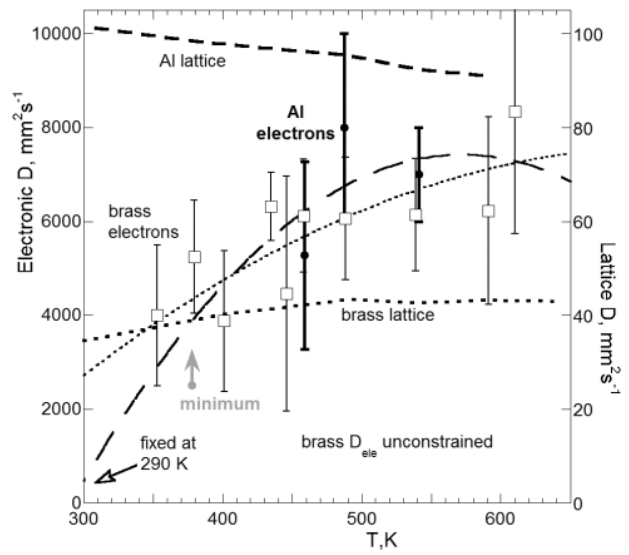

(b)

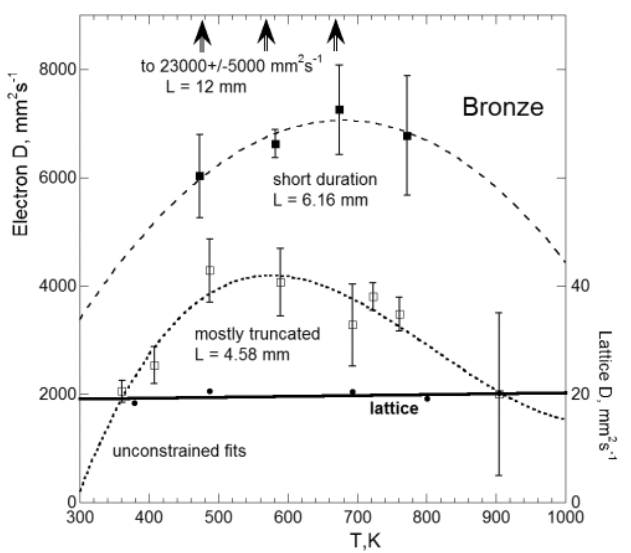

(c)

Fig. 15. Temperature dependence of $D_{\text {ele }}$ for metals and alloys with very fast signals. See Fig. 13 for most symbols. (a) High purity silver of varying lengths and conditions (listed in the legend). Significantly longer lengths have higher $D_{\text {ele }}$ whereas run conditions have little effect. Data at low $T$ were constrained by collecting $\sim 8$ shots per setpoint. (b) Aluminum and brass have similarly high $D_{\text {ele }}$, whereas their lattice contributions differ in value and slope. The gray dot is a single shot: other shots at this temperature did not fit well. This datum is not included in the fit, but indicates lower $D_{\text {ele }}$ at lower $T$. (c) Slightly magnetic Mn-bronze. The uncertain point at $875 \mathrm{~K}$ was not included in the fit. Length affecting $D_{\text {ele }}$ is substantiated, even though data from the $12 \mathrm{~mm}$ section is highly uncertain. 


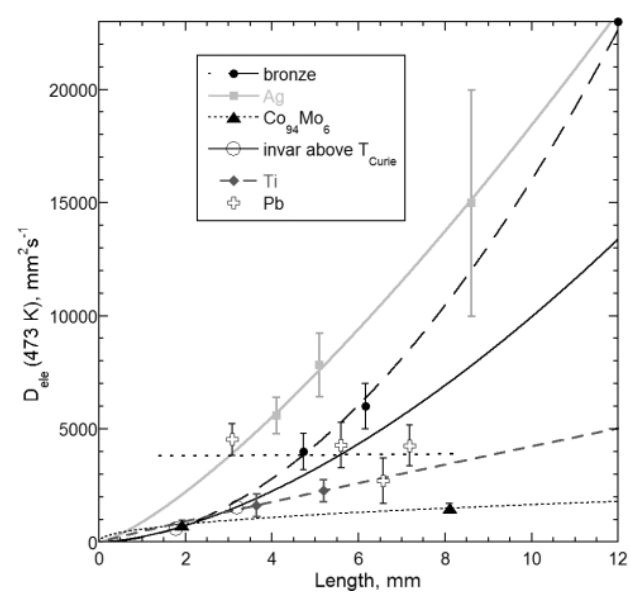

(a)

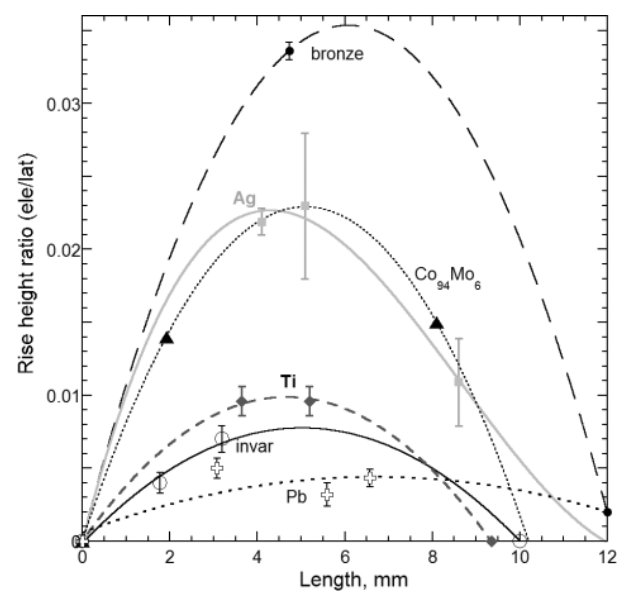

(b)

Fig. 16. Dependence of (a) $D_{\text {ele }}$ and (b) the rise height ratio on sample thickness. For bronze, $L=6.16 \mathrm{~mm}$ only involves short data collections. For all samples, the ratio approaches zero at some large $L$ of 6-14 mm, depending on composition. Power-law fits for $D_{\text {ele }}$ necessarily include the origin.

transitions. The dependence of $D_{\text {ele }}$ on $T$ and on the transitions does not appear to be affected by element purity or alloying.

The dependence of $D_{\text {ele }}$ on $T$ (Figs. 13-15) is distinct from and more complicated than the monotonic increase of the electronic/lattice rise height ratio with $T$ (Fig. 11). Similarly, $D_{\text {ele }}$ and rise height ratio depend differently on length (Fig. 16). Thus, our results on $D_{\text {ele }}$ are independent of rise height (except for the requirement that signal height exceeds the noise). Yet, the best constrained data have fits that point to $D_{\text {ele }}=0$ near $300 \mathrm{~K}(\mathrm{Mn}, \mathrm{Ni}, \mathrm{Fe}$, thin $\mathrm{Ag}, \mathrm{Pb}$, brass, and bronze) in agreement with the best constrained intercepts from rise height ratios (Table 3). This finding suggests that electronic diffusivity and rise height are affected by similar limiting factors (see below).

Thermal diffusivity for the slow rise is $\sim 0.005 \times$ that of the fast, but this proportionality varies with temperature, composition, and the presence of magnetism (Figs. 13-15; Tables 2 and 3). Mostly, for elements, $D_{\text {lat }}$ decreases with $T$, whereas for alloys $\bar{D}_{\text {lat }}$ increases with $T$. Changes in $D_{\text {lat }}$ are generally more gradual then changes in $D_{\text {ele. }}$.

Magnetic transitions affect $D_{\text {lat }}$, but this effect is neither huge nor abrupt, as observed for $D_{\text {ele }}$. Structural transitions affect $D_{\text {lat }}$ in various ways (e.g., Refs. 24 , $34,57,60$ and 62), although the figures in our report only show minor changes. Thus, the behavior of $D_{\text {lat }}$ differs substantially from that of $D_{\text {ele }}$.

From the data in Figs. 11-16 and the above discussion, the fast and slow processes diffusing heat in metals are largely independent. This finding is consistent with the nearly-free electron model. 


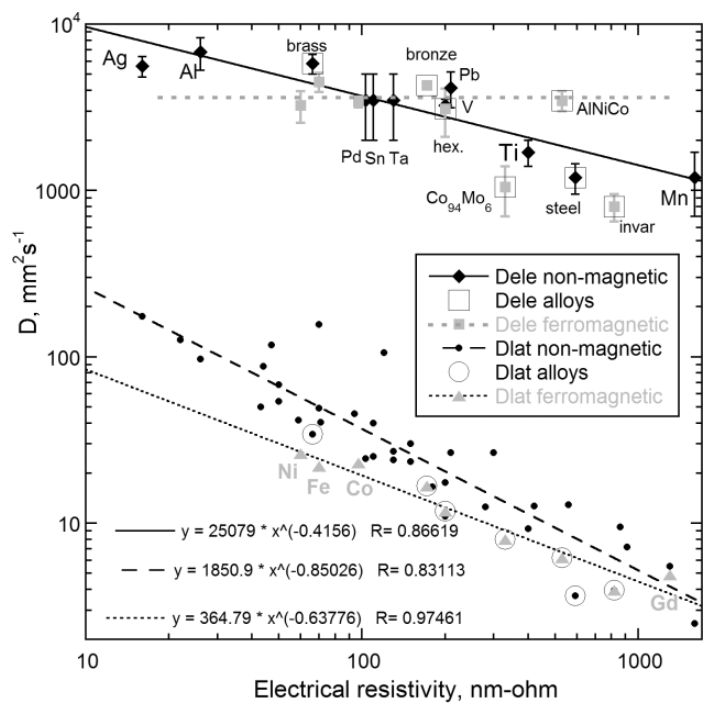

Fig. 17. Dependence of thermal diffusivity on electrical resistivity. For $D_{\text {ele }}$, the most accurate values at $473 \mathrm{~K}$ are used to avoid uncertainties associated with projection. Measurements of $\mathrm{Ni}$ and invar indicate that the nonmagnetic state has higher $D_{\text {ele }}$ than the magnetic state. For $D_{\text {lat }}$, our data (Table 2) and direct measurements of Touloukian et al..$^{22}$ are used, which are uncertain by $\sim 2 \%$ and $\sim 5 \%$, respectively. Least squares fits are listed. Electronic and lattice heat transports are affected differently by magnetism and cation disorder.

\subsection{Dependence of $D_{\text {ele }}, D_{\text {lat }}$, and rise height ratio on physical properties and an explanation of the complex dependence of $D_{\text {ele }}$ on $T$}

To investigate microscopic behavior, we compare our results to various physical properties. Due to changes in both detector characteristics and rise height ratios with temperature, the most reliable values for $D_{\text {ele }}$ are near $473 \mathrm{~K}$ (Table 3). Given the uncertainties in projecting the trends back to $298 \mathrm{~K}$, and because $D_{\text {ele }}$ at $298 \mathrm{~K}$ is likely negligible for many materials, we use our results near $473 \mathrm{~K}$ (Table 3) in comparing physical properties (Figs. 17 and 18). These trends represent ordinary temperatures.

We did not find positive trends with carrier speed or structural parameters. Fermi speed and acoustic velocities only vary by about a factor of two for the metals studied,, 24 and thus the trends in either of the thermal diffusivities, which each go roughly as the product $u \lambda$ [Eq. (15)], largely express variations in mean free path or lifetime for each carrier. As discussed in diverse sources, motions of electrons and phonons are each impeded by interactions with electrons, phonons, and defects, as well as by magnetism. Hence, the same factors should affect transport of heat by each carrier, although the specific responses should differ.

Diffusions of heat by electrons and phonons both become slower and less efficient as resistivity increases, such that $D_{\text {lat }}$ depends more strongly on resistivity, being 
nearly inverse (Fig. 17). Lattice transport is additionally hindered by magnetic interactions and cation disorder, as occurring in electrical insulators (e.g., garnets ${ }^{61}$ ). The magnetic elements define one $D_{\text {lat }}$ trend, even though several different crystal structures and electronic configurations are represented. Magnetic alloys cluster around the $D_{\text {lat }}$ trend for magnetic elements. Across the Curie transition, $D_{\text {lat }}$ is unchanged although its temperature derivative is discontinuous [Fig. 13(a), 14(b), and 14(c); see Refs. 57 and 60 for Fe].

Electronic heat transport responds to the same phenomena that influence lattice transport, but in contrasting ways. First, $D_{\text {ele }}$ depends less strongly on resistivity (for both nonmagnetic and magnetic samples), and irregularly responds to cation disorder (Fig. 17). Upon crossing the Curie transition, $D_{\text {ele }}$ increases strongly in both elemental $\mathrm{Ni}$ and the alloy invar. For invar, loss of ferromagnetism at $T_{\text {Curie }} \sim 550 \mathrm{~K}$ doubles $D_{\text {ele }}$, with the change being $\sim 800 \mathrm{~mm}^{2} \cdot \mathrm{s}^{-1}$. The change in $\mathrm{Ni}$ is even greater at $T_{\text {Curie }}=627 \mathrm{~K}$, tripling $D_{\text {ele }}$, with the change being $\sim 3500 \mathrm{~mm}^{2} \cdot \mathrm{s}^{-1}$ [these values are from averaging data, not curve fits: see Fig. 13(c)]. Magnetism may influence electronic heat transport, and is a possible explanation for $D_{\text {ele }}\left(\sim 3800 \mathrm{~mm}^{2} \cdot \mathrm{s}^{-1}\right)$ being similar for pure magnetic elements at $473 \mathrm{~K}$ (Table 3 ). However, $D$ values are scattered for magnetic alloys (Table 3; Fig. 17). Complex behavior associated with magnetism is consistent with results from ultrafast spectroscopy, which shows that excited electron lifetimes associated with magnetic interactions in ordinary ferromagnets [e.g., Ni (Ref. 12)] are slow as in phononic interactions, whereas in more complex materials (e.g., pnicnides ${ }^{11}$ ) magnetic interactions have fast lifetimes. Although investigation of additional materials is needed to fully understand the effect of magnetism on electronic heat transport, the present examples demonstrate that heat carrying electrons are affected differently by magnetism that are phonons.

An inverse correlation exists between $D_{\text {ele }}$ and $C_{\text {ele }}$ [Fig. 18(a)], which is attributed to existence of a greater number of excited electrons creating more interactions. The data indicate that electrons impede their own flow. Resistivity only roughly correlating with $C_{\text {ele }}$ [Fig. $18(\mathrm{a})$, bottom] corroborates that electronic lifetime is reduced as the number of participating electrons increase, but that other factors pertain. In ultrafast spectroscopy, lifetimes for the hot state decrease as both $C_{\text {ele }}$ and particle energies increase (Fig. 2), suggesting that $D_{\text {ele }}$ should depend on $C_{\text {ele }}$ and temperature (heat energy). In contrast, neither $D_{\text {lat }}$ nor resistivity are correlated with lattice heat capacity at $298 \mathrm{~K}$ (not shown).

Rise height ratio is affected by the same factors. Like $D_{\text {ele }}$, the rise height ratio also approaches 0 as $C_{\text {ele }} / C_{\text {lat }}$ approaches 0 for elements [Fig. 18(c)] with similar thicknesses $(3-5 \mathrm{~mm})$ and similar resistivities $(130-400 \mathrm{~nm} \cdot \mathrm{ohm})$. Rise height ratio is affected inversely by resistivity and by thickness, but only when this ratio is large.

The behavior described above is consistent with the observed temperature dependence. As temperature increases from some low value, more and more electrons 


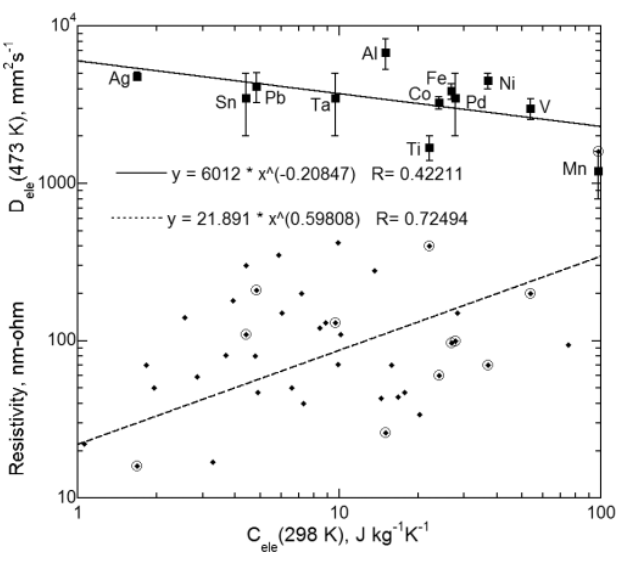

(a)

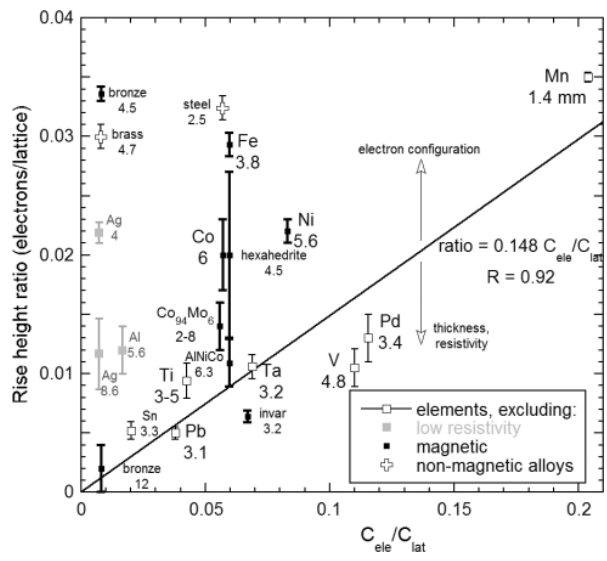

(b)

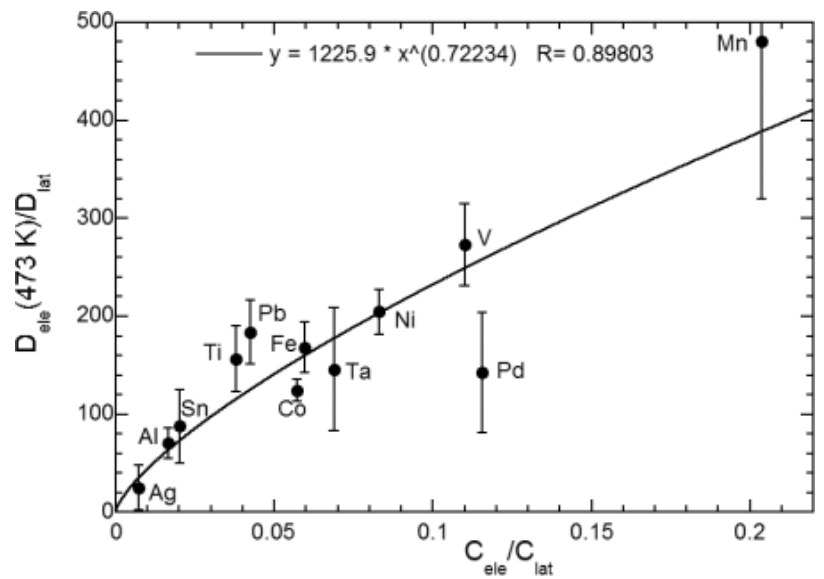

(c)

Fig. 18. Relationships of thermal diffusivity with heat capacity for elements. (a) Dependence of electronic properties of elements on $C_{\text {ele }}$. Opposite trends exist for resistivity (fit and circles for the elements studied here; triangles for all) and $D_{\text {ele }}$ (squares). Higher $C_{\text {ele }}$ means that more electrons can be excited, which creates more interactions and shorter mean free paths, and thus lower $D_{\text {ele }}$ while raising resistivity. The scatter in resistivity indicates other factors pertain, such as structure. Although we have shown $C_{\text {ele }}$ at $298 \mathrm{~K}$, changing the temperature to $473 \mathrm{~K}$ will not alter the observed linear trend because $C_{\text {ele }}=\gamma T$. (b) Ratios of heat transfer compared to ratios of heat content. If the electrons uptake a greater proportion of the heat, then the electrons transport relatively more heat. This trend is consistent with electronic heat flow being a perturbation of the lattice heat current. (c) Dependence of rise height ratio on heat capacity ratio. Labels provide sample name and thickness (in mm). For alloys (open cross), $C_{\text {ele }}$ was estimated from elemental values and the chemical composition, which may not be valid. The linear trend was constrained to include the origin and excludes elements with very low resistivity (gray symbol, $<30 \mathrm{~nm} \cdot \mathrm{ohm}$ ) and magnetic properties (filled squares), both of which have high ratios. That length reduces the ratio is evident in our Mn-bronze (which is magnetic) and silver data and suggested in the trend for the magnetic iron alloys (excepting invar, which is near its Curie point). 
are excited to higher energies (yielding higher $C_{\text {ele }}=\gamma T$ and higher speeds). At first, $D_{\text {ele }}$ increases with $T$ as the population capable of transmitting heat grows. However, once some sufficiently large electron population is excited, the carriers interfere with each other, shortening their mean free paths and lowering $D_{\text {ele }}$. This interference causes a maximum to exist in $D_{\text {ele }}$ versus $T$. Section 6 suggests an explanation for the absence of an electronic heat current below some finite, generally ambient temperature.

Alternatively, the true form for $D_{\text {ele }}(T)$ might be a skewed Gaussian, with asymptotes of $D_{\text {ele }} \rightarrow 0$ as $T \rightarrow 0$ and $\rightarrow \infty$ rather than intercepts at finite temperatures, as suggested by our polynomial fits. Our measurements are insufficiently accurate to determine the exact shape of the $D_{\text {ele }}$ versus $T$ curves. Hot electron lifetimes provide some insight. These lifetimes become independent of energy at high energy (Fig. 2; Ref. 14) and are thus independent of high electron population temperature. This is compatible with $D_{\text {ele }}(T)$ flattening to some low value at very high $T$. Our extensive data on Ni are compatible with high $T$ flattening. Regarding hot electron just above the Fermi energy, their lifetimes asymptote to infinity, ${ }^{14}$ showing stability against perturbation at room temperature, where fs-spectroscopy was conducted. Hence, the lack of detectable electronic heat currents due to small perturbations induced during our heat transfer experiments at $298 \mathrm{~K}$ is consistent with fs-spectroscopy, also at $298 \mathrm{~K}$. Heat transfer lifetimes are calculated and compared to hot electron lifetimes in Sec. 6. Magnetism provides additional interactions among electrons. All other matters being equal (i.e., at Curie points), magnetism reduces $D_{\text {ele }}$ while not affecting rise height ratio.

Long length increases $D_{\text {ele }}$ while simultaneously reducing the rise height ratio [Fig. 18(c)]. Thus, the number of carriers is lowered, causing fewer interactions, a larger mean free path, and more efficient heat transport. An obvious mechanism for reduction of carriers is irreversible conversion of electronic to phononic heat.

Rise height ratio is affected by other factors besides length and $C_{\text {ele }} / C_{\text {lat }}$. Examining Figs. 18(c) and 12(b) suggests that photon penetration is unlikely to be a factor. Band structure likely plays a role, but probing this is beyond the scope of our study.

Substances with high $C_{\text {ele }}$ have more excited electrons, more interactions, and hence lower $D_{\text {ele }}$ as well as larger electrical resistivity. Because the latter property is also tied to $C_{\text {ele }}$, albeit roughly [Fig. 18(b)] these effects are difficult to separate in our dataset which involves varying length as well. The figures suggest that disorder is unimportant to electronic heat transport compared to the other factors, because elements and disordered alloys behave similarly.

The above findings are not entirely consistent with existing formulations for an electron gas. A key factor omitted from previous models is that a competing phononic heat current exists. This competition, as well as the low heat capacity of the electron, needs to be included in a new model of metallic heat transfer (Sec. 5). The essential point is that at ordinary temperatures, the lattice uptakes the ma- 
jority of heat as it enters the metal and must transport this heat, because the electronic carrying capacity is almost negligible. Hence, the electronic contribution to heat transport is minimal, despite the high measured diffusivity of this mechanism.

\subsection{Calculation of $k_{\mathrm{ele}}$ and comparison to $k_{\text {lat }}$}

We computed $k_{\text {ele }}$ from Eq. (2) using $\gamma$ from experiments ${ }^{21}$ to provide $C_{\text {ele }}(T)$ and

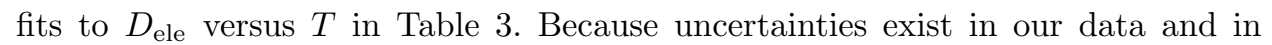
extrapolating $\gamma$ from $\sim 10 \mathrm{~K}$ to over $298 \mathrm{~K}$, using density at $298 \mathrm{~K}$ should suffice. The most accurate $k_{\text {ele }}$ values are at $473 \mathrm{~K}$ (listed in Table 3 ). The $T$ dependence is shown in Fig. 19. Values for Pd with estimated $D_{\text {ele }}$ are shown only between $300 \mathrm{~K}$ and $640 \mathrm{~K}$; Sn is also similarly treated. The nearly-linear trends of these elements are largely due to the behavior of their heat capacities. For the other elements, $k_{\text {ele }}$ is truncated in accordance with the fits to $D_{\text {ele }}$.

Values of $k_{\text {ele }}$ do not correspond with electrical conductivity [Tables 1 and 3 ; Fig. 20(a)] which is consistent with electron-electron scattering, rather than electron-phonon interactions, transferring heat. Roughly, $k_{\text {ele }}$ is 20 times $k_{\text {lat }}$ (Tables 2 and 3) which is consistent with faster speeds, but shorter mean free paths, of electrons compared to phonons. High values of $k_{\text {ele }}$ exist only for metals with many $d$-electrons in their conduction bands, whereas low values exist for elements with $s$ - and $p$-type electrons in their conduction bands. The only exception is $\mathrm{Ti}$, which has the least number of $d$-electrons, only two.

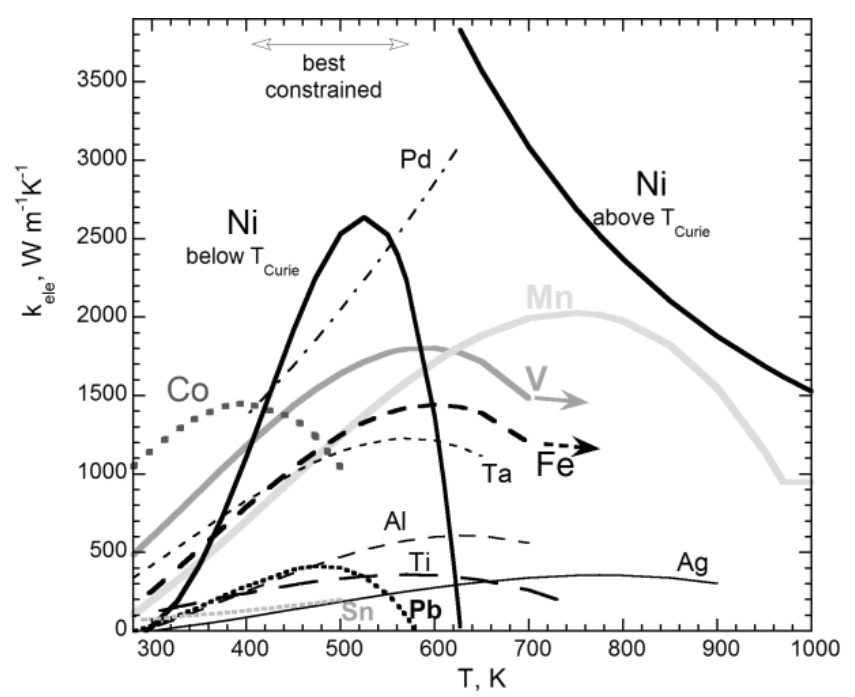

Fig. 19. Electronic thermal conductivity as a function of temperature for elements, as labeled. Nearly-linear trends exist for Pd and Sn where $D_{\text {ele }}$ was obtained over a narrow range in temperature, see Table 3. Constant density at $298 \mathrm{~K}$ was used as well as constant $\gamma$ (Table 1). Values were truncated based on trends in $D_{\text {ele }}$. For some cases, the $\sim 300 \mathrm{~K}$ value depends on the intercept from rise height ratio with $T$ (see Figs. 13-16; Table 3). 


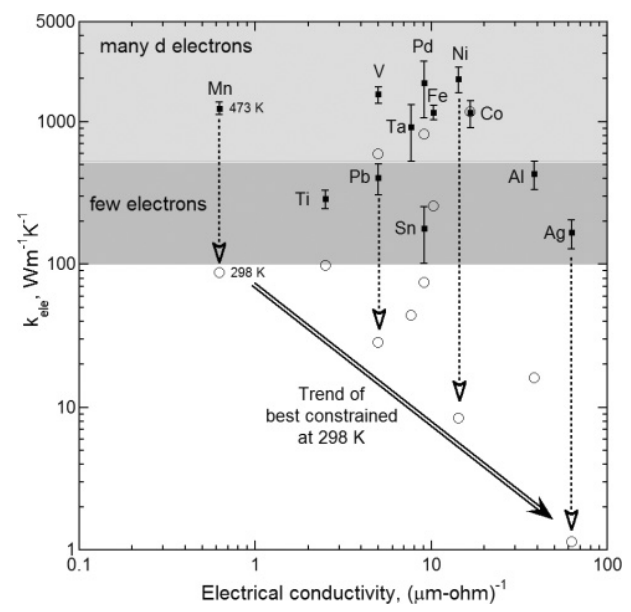

(a)

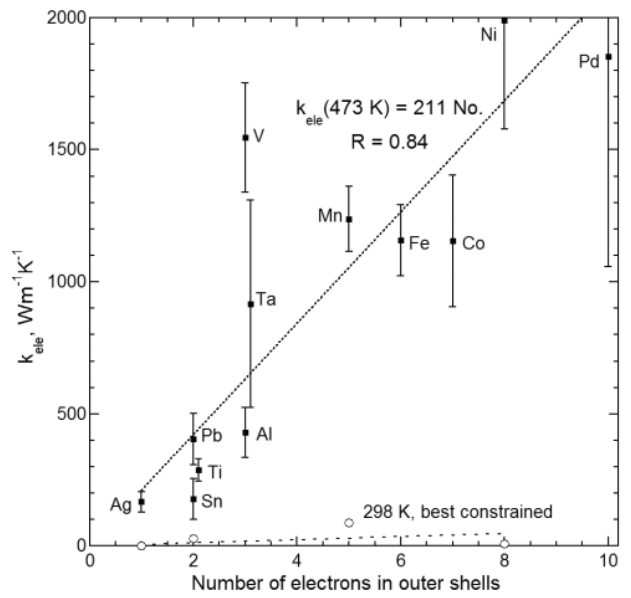

(b)

Fig. 20. Relationships of $k_{\text {ele }}$ with physical properties. For consistent comparisons, the fits to $D_{\text {ele }}$ were used to compute $k_{\text {ele }}$ at $300 \mathrm{~K}$ and $473 \mathrm{~K}$. Most samples are $3-5 \mathrm{~mm}$ thick, but the

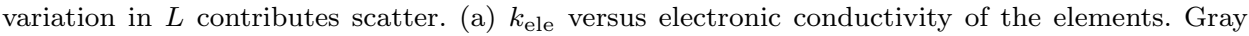
areas indicate the number of electrons in outer orbitals. At $300 \mathrm{~K}$, an inverse correlation exists

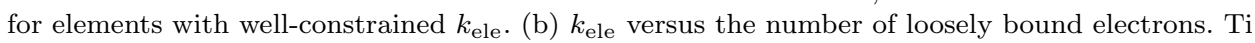
and Ta were shifted slightly to the right for clarity.

Electron configuration strongly affects $k_{\text {ele }}$ [Fig. 20(b)]. This plot suggests that the number of loosely bound electrons is the primary factor. Except for V, elements diverging from the linear trend have less certain $k_{\text {ele }}$, mainly due to noise in the $T-t$ curves. For vanadium, $\gamma$ is quite high: only $\mathrm{Mn}, \mathrm{Pd}$, and $\mathrm{U}$ have higher values on a per mole basis. ${ }^{21}$ Obtaining $\gamma$ is based on subtracting a theoretical lattice heat capacity from the experimental value plus assuming a linear response for $C_{\text {ele }}(T)$. Because $\mathrm{V}$ is superconducting at relatively high temperature $[5 \mathrm{~K}$ (Refs. 21 and $24)$ ], constant $\gamma$ for $\mathrm{V}$ may not accurately represent $C_{\text {ele }}$.

Steady-state measurements do not provide such high $k$ for pure metals (Table 2; Refs. 23-25). Previous experiments record lower $k$ associated with the lattice because the excited electrons rapidly reequilibrate (Figs. 6 and 8-10) and do not contribute to heat flow measured more than a few ms after application of the heat pulse (quasi-steady-state conditions). To better understand thermal behavior of metals, the next section develops a model for heat transfer involving multiple mechanisms.

\section{Model}

Measurements designed to distinguish the electronic component from the lattice component of thermal conductivity in metals were not previously attempted for a variety of reasons. Chief among these is the expectation of a single thermal event, even in transient experiments, related to the believed existence of an effective sin- 
gle conductivity, regardless of carrier speeds differing. This assumption is embodied in Eq. (10). For metals, thermal evolution is complex (Figs. 3-6), and $T-t$ curves present two rises, one for each process, which are independent, in accordance with the nearly-free electron model, and sequential, due to vastly different speeds characterizing electrons and phonons. Because it is impossible to exactly characterize such sequential behavior with a single conductivity, we define an equivalent conductivity $\left(k_{\text {equivalent }}\right)$ as an approximate value which conserves energy while representing the measured $T-t$ curve as consisting of a simplified, single rise.

Attribution of the majority of heat transport to electronic processes has not previously been questioned due to reasonable agreement of the WF model [Eq. (5); Fig. 1] with measured thermal conductivity at $298 \mathrm{~K}$, coupled with Meissner's ${ }^{42}$ assertion that thermal conductivities simply sum [Eq. (10)]. This historical development, along with the presence of spark noise in previous experiments, resulted in incorrect interpretation of available thermal curves, such that the small, initial electronic component (e.g., Figs. 3-7) has been overlooked while the main, lattice component was attributed to electronic processes. This section addresses why $k_{\text {ele }}$ is significantly higher than previously predicted (Fig. 1), yet the amount of heat transferred by this process is much smaller than previously assumed, by developing new summation rules for thermal conductivity, which are based on energy conservation.

To place our approach in context, previous models of the microscopic origin of thermal conductivity $(k)$ assume steady-state conditions..$^{76}$ This presumption of thermal equilibrium is inappropriate for describing the dynamic process of thermal reequilibration, as explicitly occurs during transient measurements such as LFA and the commonly used (modified) Ångstrom technique, ${ }^{22}$ and implicitly precedes collection of data during steady-state measurements. Although Eq. (10) is believed to hold under a wide range of conditions, its validity is rather limited, as demonstrated by considering Kirchhoff's law which sums heat currents:

$$
q=-k \frac{\partial T}{\partial x}=-\left(k_{1} \frac{\partial T_{1}}{\partial x}+k_{2} \frac{\partial T_{2}}{\partial x}\right)=q_{1}+q_{2} .
$$

For Eq. (10) to hold, all temperature gradients in Eq. (18) need to be identical. Either

$$
\frac{q_{1}}{k_{1}}=\frac{q_{2}}{k_{2}}
$$

or only one mechanism operates (i.e., either $k_{1}=k_{2}$ or $k_{2}=0$ ). Equation (19) shows that for Eq. (10) to hold for metals, electronic and phononic processes cannot independently move heat. Without going into detail, it should be clear that Eq. (19) links heat capacities to thermal conductivities in a manner that is not dictated by physical properties, e.g., the mechanism with higher $k$ must have higher $C$ at all temperatures for Eq. (19) to hold. This is not the case for electrons and phonons. ${ }^{4,6,7}$ Thus, Eq. (10) is not generally true, and seems especially inapplicable to metals above room temperature. 
To describe heat transfer, this section models the approach to equilibrium during time-dependent heat flow under adiabatic conditions. Our analysis depicts onedimensional flow under a nonnegligible $T$-gradient, which describes experiments on solids. A general rule is derived for independent mechanisms, and is solved for several limiting cases. We then derive another general rule to describe a single mechanism with multiple carriers. We also discuss the behaviors of phonons and electrons, and explain how their microscopic behaviors influence heat transfer and affect our sum rules.

The equations presented below will be combined with our new data (Sec. 4) and available information on carrier speeds to quantify phonon and electron lifetimes and mean free paths in Sec. 6 .

\subsection{Derivation of sum rules for independent mechanisms}

To model transient behavior, we consider nearly-instantaneous application of a prescribed amount of heat $(Q)$ to a bar of material with initial temperature $\left(T_{0}\right)$, area $=A$, length $=L$, and volume $(V=A L)$. Parallel heat flow involving simultaneous, but independent, mechanisms is described by multiple bars of equal density wherein only one mechanism operates in each bar (Fig. 21).

We emphasize that distinct temperature fields are a consequence of noninteracting mechanisms at disequilibrium, which immediately follows a thermal disturbance, as exemplified by ultrafast spectroscopic studies of hot electrons. ${ }^{9-17}$ Conserving energy requires that the apparent temperature field is a weighted sum of temperature fields associated with each mechanism in the differing bars:

$$
C T=C_{1} T_{1}+C_{2} T_{2}=\Sigma C_{i} T_{i}
$$

Assuming that the independent mechanisms obey Fourier's law leads to

$$
k \frac{\partial T}{\partial x}=k_{1} \frac{\partial T_{1}}{\partial x_{1}}+k_{2} \frac{\partial T_{2}}{\partial x_{2}}+k_{n} \frac{\partial T_{n}}{\partial x_{n}}=\sum k_{i} \frac{\partial T_{i}}{\partial x_{i}},
$$

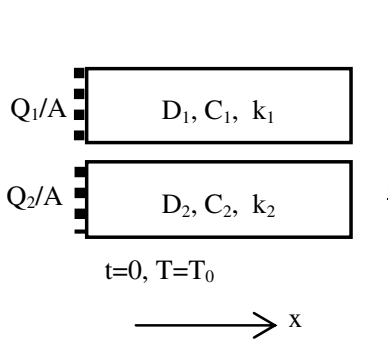

(a)

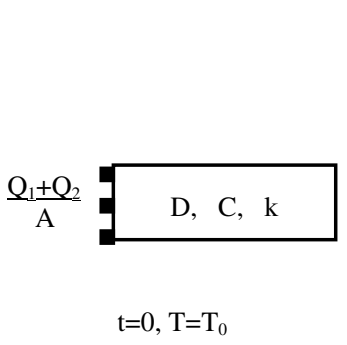

(b)

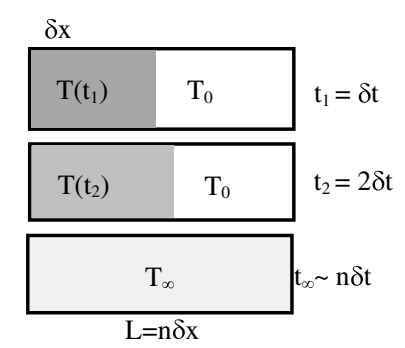

(c)

Fig. 21. Schematic of heat flow in parallel. (a) Initial conditions in two parallel bars. Heavy dots represent application of the instantaneous pulse. (b) Initial conditions for a blended bar. (c) Time-evolution during an adiabatic process. 
where the subscripts indicate which bar the mechanisms operate in. Combining Eqs. (20) and (21),

$$
k \sum C_{i} \frac{\partial T_{i}}{\partial x_{i}}=C \sum k_{i} \frac{\partial T_{i}}{\partial x_{i}} .
$$

Solving Eq. (22) requires making additional assumptions, which is possible through considering a few limiting cases.

Case 1 relates to cryogenic temperatures in metals. In case 1 , we assume that the thermal diffusivity $(D=k / C)$ is similar in both bars, resulting in a single thermal rise, and an equivalent conductivity due to governance by Eq. (9). Hence, their temperature evolves similarly with distance and time, providing thermal equilib$\operatorname{rium}\left(T \approx T_{1} \approx T_{2}\right.$ ) as reequilibration progresses. Blending the two bars provides a single medium [Fig. 21(b)]. Considering incremental changes at quasi-equilibrium [Fig. 21(c)], and conserving energy:

$$
T_{1}=T_{0}+\frac{Q_{1}}{C_{1} A \delta x_{1}} ; \quad T_{2}=T_{0}+\frac{Q_{2}}{C_{2} A \delta x_{2}} ; \quad T=T_{0}+\frac{Q}{C A \delta x} .
$$

If $C_{1} \approx C_{2}$ then $k_{1} \approx k_{2}$ and $Q_{1} \approx Q_{2} \approx Q / 2$. The last stipulation conserves energy, and insures that the final temperatures associated with the two mechanisms are similar. For two mechanisms, $C=C_{1}+C_{2}$. Combining these stipulations with Eq. (23) gives

$$
\frac{C}{2} \delta x \approx C_{1} \delta x_{1} \approx C_{2} \delta x_{2}
$$

From Figs. 21(a) and 21(b), heat flux [ $q=Q /\left(A t_{p}\right)$, where $t_{p}$ is pulse duration], as applied to the blended bar, equals the sum $q_{1}+q_{2}$. Letting $\partial T / \partial x=\delta T / \delta x$ and $\partial T=\delta T \approx \delta T_{i}$ relates the slopes of the temperature fields such that

$$
n \frac{C_{i}}{C} \frac{\partial T}{\partial x} \approx \frac{\partial T_{i}}{\partial x_{i}}
$$

Applying Eq. (25) to the summation of heat currents [Eq. (18)] results in

$$
k \frac{\partial T}{\partial x}=k_{1} \frac{\partial T_{1}}{\partial x_{1}}+k_{2} \frac{\partial T_{2}}{\partial x_{2}}=k_{1} \frac{2 C_{1}}{C} \frac{\partial T}{\partial x}+k_{2} \frac{2 C_{2}}{C} \frac{\partial T}{\partial x}=\sum k_{i} \frac{n C_{i}}{C} \frac{\partial T}{\partial x} .
$$

For $n$ independent mechanisms, $C=\Sigma C_{i}$, so

$$
k=n \frac{\sum C_{i} k_{i}}{\sum C_{i}} \text { for } C_{i} \approx C_{j} \text { and } D_{i} \approx D_{j}
$$

For the more restrictive condition of $C_{i}=C_{j}$, then Eq. (27) reduces to $k=\Sigma k_{i}$ as in Eq. (10) but only under the restriction of $D_{i} \approx D_{j}$.

The above (case 1 ) describes a single rise for a $T-t$ curve that embodies both mechanisms. When the $C_{i} \mathrm{~s}$ and $D_{i} \mathrm{~s}$ are each similar, the curve represents the average behavior of the various carriers. For this averaging to occur requires either accidental similarity of the physical properties or that the processes exchange energy (i.e., are not independent), thereby creating similar properties. 
Case 2 assumes similar $D_{i}$ s as in the previous analysis, but we relax the stipulations on relative $C_{i}$ values, and, importantly, allow the temperature fields to vary relative to one another. Applying Eq. (25) to each side of Eq. (22) results in

$$
k \sum C_{i} \frac{n C_{i}}{C} \frac{\partial T}{\partial x} \cong C \sum k_{i} \frac{n C_{i}}{C} \frac{\partial T}{\partial x} .
$$

Canceling like terms, using $C=\Sigma C_{i}$, and rearranging Eq. (28) gives

$$
k \cong \sum k_{i} C_{i} \frac{\sum C_{i}}{\sum C_{i}^{2}} .
$$

If $C_{i}=C_{j}$, then Eq. (29) reduces to $k=\Sigma k_{i}$ as did Eq. (27). Equation (29) relates $k$ to $C$ by assuming a relationship between the spatial derivative of independent temperature fields and heat capacity.

Equations (27) and (29) for noninteracting mechanisms diverge from Meissner's sum, Eq. (10). The weighting factors revealed by our derivations account for energy conservation and effects of transient phenomena in heat flow. Equation (10) incorrectly describes heat transfer, except for special cases involving equivalent diffusivities. Furthermore, Eqs. (27) and (29) are not limited to metals at cryogenic temperatures, but apply to any heat transfer problem with multiple mechanisms with similar diffusivity.

Case 3 departs from the above by assuming that the specific heats of each mechanism differ significantly, such that $C_{1} \ll C_{2} \approx C$ resulting in $D_{1} \gg D_{2}$, for $k_{1}>$ or $\sim k_{2}$. This important case represents behavior of metals at ordinary temperatures (i.e., $T$ above some tens of kelvins). Such disparate thermal diffusivity values will cause the temperature-distance evolution associated with each process to differ greatly. Over a relatively short time, fast process 1 will equilibrate to its final $T_{\infty}$ [Fig. 21(c)]. Because bar 1 representing process 1 is isothermal, $\partial T_{1} / \partial x_{1}=0$. However, over this same time interval, $T_{2}$ in bar 2 still changes with distance, providing a heat current of $k_{2} \partial T_{2} / \partial x_{2}$. Summing currents gives $k \partial T / \partial x=k_{2} \partial T_{2} / \partial x_{2}+0$ and thus, $k=k_{2}$.

We now address case 3 more rigorously. Because energy is conserved, $T_{\infty}$ for process 1 equals that of process 2 and of the blend, which is computed from Eq. (18) by setting $\delta x=\delta x_{1}=\delta x_{2}=L$. Consequently, $Q_{1} /\left(C_{1} V\right)=Q_{2} /\left(C_{2} V\right)=Q /(\overline{C V})$ from Eq. (23). (Although fast process 1 in the blended bar might initially absorb more heat than expected from thermal equilibrium, i.e., $Q^{*}>Q_{1}=Q C_{1} / C_{2}$, carriers of type 1 must subsequently transfer this excess $\left(Q^{*}-Q_{1}\right)$ to carriers of type 2 , in order to attain isothermal conditions at very long times.) Because $Q_{1}=Q C_{1} / C_{2}, Q_{1}$ is negligible compared to $Q_{2}$, then $k_{1} \partial T_{1} / \partial x_{1}$ is negligible compared to $k_{2} \partial T_{2} / \partial \mathrm{x}_{2}$. Summing heat currents gives

$$
k=k_{2}, \quad \text { for } C_{1} \ll C_{2} .
$$

Importantly, the weighted sum of Eq. (29) reduces to Eq. (30) if $C_{1} \ll C_{2}$, showing that Eq. (29) is more general than the restricting conditions used in the derivation would suggest. In contrast, Eq. (27) forces the temperature fields to be the same, 
and thus only occurs under special circumstances for independent mechanisms, but it is generally applicable for a single mechanism with multiple carriers (Sec. 5.2).

For case 3 , wherein $D_{1} \gg D_{2}$, mechanism 1 proceeds over a much faster rate, based on Eqs. (9), (14), and (15) because $L$ is the same for both processes (Fig. 21). Hence, $u_{1} \gg u_{2}$. But, mechanism 1 carries little heat $\left(C_{1} \ll C_{2}\right)$. Hence, during reequilibration, as shown in $T-t$ curves, the fast mechanism is expressed as a small rise at short times, and thus, the fast mechanism 1, which carries little heat, has little or nothing to do with long-term behavior. For this reason, Eq. (30), which is identical to

$$
k=k_{\text {lat }} \text { for metals, }
$$

reasonably describes the observed behavior of electrons and phonons in metals, down to some tens of kelvins, as discussed further in Sec. 6 .

Case 3 limited the range of $k$. To consider all possible values of $k$, we now assume that $k_{1}<k_{2}$ while $C_{1}<C_{2}$. In this variant, both the amount of heat and how fast it is transported by mechanism 1 are negligibly small, so that this mechanism has a negligible effect on heat transfer. The material property is again represented by Eq. (30).

In summary, specific heat is an equally important parameter as thermal conductivity in describing heat transfer in a material with multiple mechanisms. As a consequence, the slower process dominates should it have a significantly higher specific heat. Why this connection exists is discussed below. It should be stressed that utilization of the above equations may not capture all aspects of a given $T-t$ curve, such as the small rapid rise observed in metals (Figs. 3-7). The rapid rise must be modeled as a time-dependent phenomenon using Eq. (9). Importantly, because our analysis covers the initial conditions up to thermal equilibration, it describes the approach to steady-state, and thus describes diverse experimental measurements of thermal conductivity. Our model is not limited to transient phenomena, but describes heat conduction when energy is conserved.

\subsection{Derivation of a sum rule for a single mechanism with multiple carriers}

For a single mechanism with a total thermal conductivity of $k_{\text {single }}$, then $C_{\text {single }}=$ $\Sigma C_{i} / n$. For a single mechanism, the carriers interact and a single temperature field is a good approximation. For this reason, case 1 is appropriate. Using the above definition while following the procedure leading to Eq. (27) gives

$$
k_{\text {single }}=\frac{\sum C_{i} k_{i}}{\sum C_{i}} .
$$

If heat capacities of the various carriers are nearly equal, then

$$
k_{\text {single }}=\frac{\sum k_{i}}{n} \equiv\left\langle k_{i}\right\rangle \quad \text { for } C_{i} \approx C_{j},
$$


where the brackets designate an average. For the end-member case of a single mechanism with only one type of carrier, Eq. (32) reduces to $k_{\text {single }}=k_{i}$, which is obviously correct.

Our results for a single mechanism differ from the kinetic theory of gas, which provides

$$
k_{\text {gas }}=\frac{\sum u_{i} C_{i} \lambda_{i}}{3 n} \equiv \frac{\sum k_{i}}{3 n} \equiv \frac{\left\langle k_{i}\right\rangle}{3},
$$

where $\lambda$ is mean free path. The reasoning behind this additional factor of 3 in the kinetic theory of gas is evident in the step-by-step derivation provided by Reif. .66 The factor of $1 / 3$ originates to the assumption that in some volume, $1 / 3$ of the molecules are moving in each of the $x-, y$-, and $z$-directions: this partitioning is recognized as being approximate. ${ }^{76}$ This situation describes small excursions from steady-state conditions with a low temperature gradient, which is required to prevent convection of gas. Early experiments and theory both revolved around these conditions, to understand the purely conductive state. Despite the limitations of the historic approach, the described fluctuations of molecules in all three directions indeed describe gas behavior. In contrast, for a solid, fluctuations are extremely restricted, permitting a steep temperature gradient to be imposed. From thermodynamic law, heat can only flow down this temperature gradient, as is evident in experiments on heat conduction which measure the flow of heat in one direction, either radially (e.g., Ref. 77) or along one Cartesian direction.

Our sum rule describes the known unidirectional nature of heat flow in solids (Figs. 5 and 21) during disequilibrium. The factor of $1 / 3$ derived for gases under steady-state conditions incorrectly describes solids, which can sustain steep thermal gradient and differ greatly from gases because in the latter, mass transport is indistinguishable from heat transport. It should be omitted from Eq. (33) and all other mechanistic models of microscopic processes involving heat transfer in solids, including the WF law, Eq. (5).

\subsection{Stipulations regarding lattice conduction sums}

Phonon lifetimes have been estimated using Grüneisen parameters (reviewed by Ref. 78). Our sum rule for a single mechanism [Eq. (31)] parallels behavior of the thermodynamic Grüneisen parameter:

$$
\gamma_{\mathrm{th}}=\frac{\sum C_{i} \gamma_{i}}{\sum C_{i}}
$$

where $\gamma_{i}$ describes a vibrational mode. ${ }^{79}$ Description of adiabats by

$$
\left.\gamma_{\mathrm{th}} \equiv \frac{\partial \ln E}{\partial \ln V}\right|_{T}=\left.\frac{\partial \ln T}{\partial \ln \rho}\right|_{S},
$$

where $E$ is the internal energy and $S$ is entropy, links $k_{\text {lat }}$ to $\gamma_{\text {th }}$. Consequently, Eq. (34) supports the form of our sum rule of Eqs. (27) and (31) for lattice transport. 
Weighting mode contributions to $k_{\text {lat }}$ by their heat capacities in Eq. (31) affects not only the relative importance of the different modes present in a solid, but also how this distribution changes with temperature. In addition, not all types of vibrational modes contribute equally to heat flow, as follows.

Mode Grüneisen parameters $\left(\gamma_{i}\right)$ are defined in terms of volume of the vibrating unit, not in terms of the volume of the unit cell. ${ }^{80}$ Hence, $\gamma_{i}$ of the transverse acoustic modes under ordinary conditions is undefined (rather than infinite ${ }^{81}$ ) because these motions generally produce only a negligible volume change. ${ }^{82}$ In rare cases, e.g., when thermal expansion is negative at low $T$, transverse modes may cause significant volume changes via bond bending as in silica glass, but this special circumstance is not germane to our high- $T$ study. Regarding optic modes, volume changes exist only for certain vibrations. From Eqs. (34) and (35), modes which involve a volume change will contribute to heat transfer. Consequently, the summation for $k_{\text {lat }}$ is dominated by the longitudinal acoustic (LA) mode and some of the optic modes:

$$
k_{\text {lat }}=\frac{\sum u_{i} C_{i}^{2} \lambda_{i}}{\sum C_{i}} \quad \text { over LA and other volume-changing modes. }
$$

The simple bcc and fcc structures that describe many metals lack optic modes ${ }^{6}$ providing

$$
k_{\text {lat }}=u_{p} C_{\text {lat }} \lambda_{\mathrm{LA}} ; \quad D_{\text {lat }}=u_{p} \lambda_{\mathrm{LA}}=u_{p}^{2} \tau_{\mathrm{LA}}
$$

where $u_{p}$ is the velocity of the compression wave (i.e., the longitudinal acoustic mode) and $\tau$ is the mean free lifetime. The hcp structure has an additional Raman mode $^{83}$ whereas the more complicated structures have many modes. Because group velocities of optic modes are small, their contribution is much smaller than the acoustic modes, and can reasonably be neglected (see Refs. 6 and 49). Even if transverse modes have an effect, their velocities are smaller than but similar to $u_{p},{ }^{7,24,82}$ and thus this detail will not change our findings. Equation (37) is used in Sec. 6 to compute mean free paths in an equivalent manner for all metals, and shoul $\mathrm{d}$ be reasonably accurate for all but the highly distorted and disordered metals. Consistent trends support the above approximations.

\subsection{Electronic thermal conductivity}

Section 1.3 explains why electron-electron scattering is the mechanism by which electrons move heat. Electron-phonon events exist, but these interactions serve to terminate heat flow by electrons via reequilibrating the electron gas with the lattice (Fig. 3). Because the amount of heat carried by the electrons is extremely small, such resorption events play a negligible role in heat transport within metals.

Conserving energy during heat transport by electron-electron scattering gives

$$
k_{\text {ele }}^{*}=u_{\text {Fermi }} C_{\text {ele }} \lambda_{\text {ele }}^{*} ; \quad D_{\text {ele }}=u_{\text {Fermi }} \lambda_{\text {ele }}^{*}=u_{\text {Fermi }}^{2} \tau_{\text {ele }}^{*},
$$


where ${ }^{*}$ indicates that a thermal field pertains. The sum is not needed because average properties are a reasonable representation. The Pauli exclusion principle requires speeds near the Fermi velocity, which is a substantial fraction of the speed of light, thereby mandating that electronic heat conduction is extremely brief compared to lattice conduction. This principle furthermore strongly restricts uptake of heat by electrons, as such the amount of heat carried by electronic processes is insignificant when compared with phononic mechanisms. The unknown property is electronic thermal lifetime or mean free path. As in lattice transport, lifetimes or mean free paths are dynamic properties which are inherently complex. The available WF model, relating electronic conductivity to heat conduction, is inapplicable to conduction band electrons which nimbly avoid the motions of the massive and slow cations.

A physical model describing mean free paths of conduction electrons during heat transfer would need to address effects of temperature, resistive attenuation, band structure, population of excited states, and magnetic interactions. This effort is beyond the scope of this paper. However, we can set some limits. A minimum $\lambda$ is provided by interatomic distances of $\sim 0.2 \mathrm{~nm}$ : lower values indicate electrons that are localized, and thus cannot effectively transport heat. A maximum $\lambda$ is set by the spacing of defects or impurity ions, which provides a means for the excited electron to shed its excess heat to the lattice. Hence, alloying significantly reduces $D_{\text {ele }}$ and $k_{\text {ele }}$. Because magnetism involves interactions of electrons with spins, this phenomenon should also reduce $D_{\text {ele }}$.

\section{Discussion}

Prior to our investigation, thermal conductivity data on metals were interpreted based on two assumptions. $\frac{3}{-}$ The first is that $k$ of a solid is the simple summation of component conductivities. The second assumption is that heat transport in metals is dominantly electronic, and secondarily phononic. Our measurements and model, along with previous data, reveal flaws in both assumptions. Assuming that electronic thermal transport dominates rests on the Wiedemann-Franz law, which traces its origins to classical thermodynamics, and has been updated several times. ${ }^{4,6,7}$ However, incremental model development can obscure errors in model underpinnings. Section 1.1 discusses how the tradeoffs in the $u_{i} C_{i}$ product underlying the WF law make this formulation ambiguous. ${ }^{29}$

Our $T-t$ curves show two independent, sequential rises in $T$, corresponding to a small amount of heat being rapidly diffused while the majority of the heat moves at a much slower pace. Radiative processes are discounted because (thick) metals are opaque to light and because the intensity of the rapid signal does not increase strongly with $T$ (Sec. 4.2), which logically dictates that the diffusional processes producing the two sequential rises arise from electronic (fast) and lattice (slow) components, respectively (Sec. 4.5). Moreover, the relative signal heights are consistent 
with known relative heat capacities. Sections 6.1 and 6.2 quantitatively analyze the measured thermal diffusivities (presented in Sec. 4) from these separate, sequential rises in $T$ using our model (Sec. 5). Because (repetitive) diffusional processes are probed, lifetimes far smaller than measurement times can be inferred using Eqs. (14) and (15). Importantly, lifetimes extracted from LFA data describe the mostly independent processes of warming the electrons and phonons down the temperature gradient by interactions with like carriers, whereas lifetimes from ultrafast spectroscopy describe cooling of excited electrons by various available processes. The processes differing for the electrons means the lifetimes from the two measurements need not be identical.

\subsection{Lattice conduction in metals: Mean free paths and lifetimes}

Thermal diffusivities from the slow rise $\left(D_{\text {lat }}\right)$ have, in general, a $3 \%$ uncertainty for the short samples, and are within a few percent of literature values of $D$ (from LFA and other techniques; e.g., Ref. 22) and of computation of $D$ from $k_{\text {meas }}$ (Table 2 ; Ref. 24). Larger differences of $\sim \overline{10} \%$ occur for nonideal conditions involving overly long collection times and $L>d$. Thus, thermal diffusivity from LFA records the same, long-term values as other quasi-steady-state measurements of thermal conductivity. Our model [Eq. (27)] indicates that lattice conduction dominates heat transport in metals when energy is conserved and at long times for ordinary temperatures $(>\sim 100 \mathrm{~K})$. This deduction is supported by calculation of mean free paths at room temperature and by comparing these calculations to interatomic distances, recognizing that the mean free path must be longer than the interatomic distance in order for heat to flow effectively across a sample.

Figure 22 is based on our new values of $D$ from the slow rise for $\mathrm{W}$ and $\mathrm{Cu}$, supplemented by $D$ calculated from preexisting data on $k$ and $C .{ }^{24}$ For C, Si, and Ge, we use measurements of $D_{\text {lat }}$ from which radiative transfer effects were removed. $\underline{33}$

Figure 22(a) explores a phononic mechanism for quasi-steady-state conduction by computing $\lambda_{\mathrm{LA}}=D / u_{P}$ [Eq. (15)] from $D=k c$, where $u_{P}$ was constrained from compiled elastic moduli. ${ }^{24}$ Foremost, all $\lambda_{\mathrm{LA}}$ values exceed the average interatomic distances (computed from molar volumes and density in the database), although in highly distorted Mn and the metalloid Te, the excess is small. Second, linear trends exist for metals with similar structures (discussed further below). Third, nonmetals (B, C, Si, and Ge), for which heat conduction is phononic, have $\lambda_{\mathrm{LA}}$ similar to metals [Fig. 22(a)]. These trends (detailed below) and values in Fig. 22(a) are consistent with lattice conduction dominating metals near $298 \mathrm{~K}$.

In Fig. 22(b), we test whether an electronic mechanism is possible for the slow, primary rise by computing $\lambda_{\text {ele }}$ from measured $D$ (i.e., the slow rise) as above, except that we now use $u_{\text {Fermi }}$ for Fermi spheres. $\frac{6}{6}$ These mean free paths are significantly shorter than interatomic distances, which should prohibit heat flow, and thereby 
shows that previous results for thermal diffusivity and thermal conductivity of a metal do not describe electronic processes. A Fermi sphere reasonably approximates Fermi surfaces of the alkali and noble metals ${ }^{4}$ and Ca. .44 Band structure calculations, including evaluation of angle-resolved photoemission experiments, $\frac{85}{2}$ give $u_{\text {Fermi }} \sim$ $0.3 \times 10^{6} \mathrm{~m} \cdot \mathrm{s}^{-1}$ for $\mathrm{Ti}, \mathrm{Ni}, \mathrm{Co}$, and the $\mathrm{Fe}-\mathrm{Ni}$ alloy permalloy, which is a factor of $\sim 4$ lower than that associated with Fermi spheres for these elements. However, even with a four fold increase, calculated mean free paths remain significantly below interatomic distances. Thus, Fig. 22(b) shows that previously measured $D$ (and thus $k$ ) values at $298 \mathrm{~K}$ are too small to result from electronic heat transport with velocities near $u_{\text {Fermi }}$, as assumed in previous models.

A phononic mechanism for the slow rise is corroborated by mean free paths depending on crystal structure [Fig. 22(a)]. For each of the alkali and noble metal series, which both have a single $s$-type electron above closed shells leading to simple structures, stretching the lattice increases the distance phonons travel between collisions, as expected. The bcc series occurring to the right of the fcc series in Fig. 22(a) is consistent with bcc being more damped and less compact (since bcc has 14 nearest neighbors whereas fcc has 12 nearest neighbors). Overall, bcc structures have shorter $\lambda_{\text {LA }}$ values than do fcc [cf. dots to open squares in Fig. 22(a)]. Generally HCP structures have low $\lambda_{\mathrm{LA}}$, which is attributed to the presence of Raman modes which could interact with acoustic modes, thereby shortening $\lambda_{\mathrm{LA}}$. Similar behav-

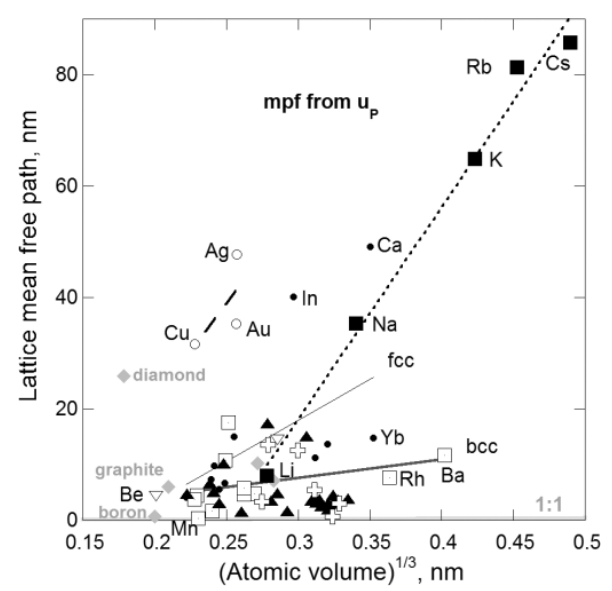

(a)

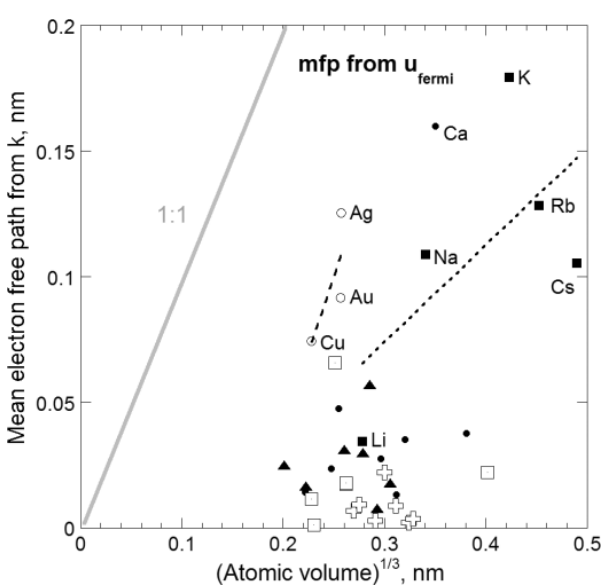

(b)

Fig. 22. Comparison of mean free paths calculated from $\lambda=k /(C u)$ at $298 \mathrm{~K}$ to the mean interatomic distance. Physical properties are from Ref. 24, except that $D$ for C, Si and Ge nonmetals (gray diamonds) are from Ref. 33. Circles and dashed line fit $=$ fcc noble metals. Dots $=$ fcc and slightly distorted fcc. Filled square and dotted line fit $=$ bcc alkali metals. Open square $=$ other bcc. Various triangles $=$ hcp. Open plus $=$ distorted structures. Thick gray line $=1: 1$ correspondence. (a) Phononic mechanism assumed, for which $u=u_{P}$. (b) Electronic mechanism assumed, for which $u=u_{\text {Fermi }}$ (data from Table 3 and Refs. 4 and 6). Unreasonably short mean free paths are obtained, refuting electrons as the main carrier of heat in metals. 
ior has been observed for insulators, wherein a strong inverse correlation exists between $D$ values and the number of infrared modes. ${ }^{33,86}$ Notably, semiconducting elements (B, graphite, Si, and Ge) cluster with most metals, whereas diamond, which has solely phononic transport, clusters with the noble metals, which most closely approach independence of electrons from phonons.

Figure 22 proves that heat conduction measurements over long times in metals at ordinary temperatures probe phononic processes, consistent with Eqs. (30a), (37), and (38). This conclusion holds whether the historical and inappropriate factor of $1 / 3$ from gas theory (see Sec. 5.2) is included or not and whether Fermi spheres or band structure calculations are used for $u_{\text {Fermi }}$. Adherence to Eq. (30) supports the validity of Eq. (29), which was derived for independent mechanisms, and shows that case 1 [Eq. (27)], which is valid for mechanisms wherein carriers exchange significant amounts of energy, does not describe the largely independent electronic and phononic heat transfer processes in metals.

Phonon lifetimes are a fundamental property of the material. Phonon lifetimes during heat flow are $\sim 0.1-100$ ps (Fig. 23). For $\mathrm{Au}, \tau_{\text {lat }}=9.7$ ps is larger than $\sim 1$ ps observed spectroscopically for electron-phonon equilibration, ${ }^{9,14,87}$ which is in reasonable agreement, due to differences in measured processes, as well as large experimental uncertainties in spectroscopic results, for times above $\sim 1$ ps. The inverse of phonon lifetimes is effectively a damping coefficient which decreases with interatomic spacing (Fig. 23). The decrease in damping is consistent with longer

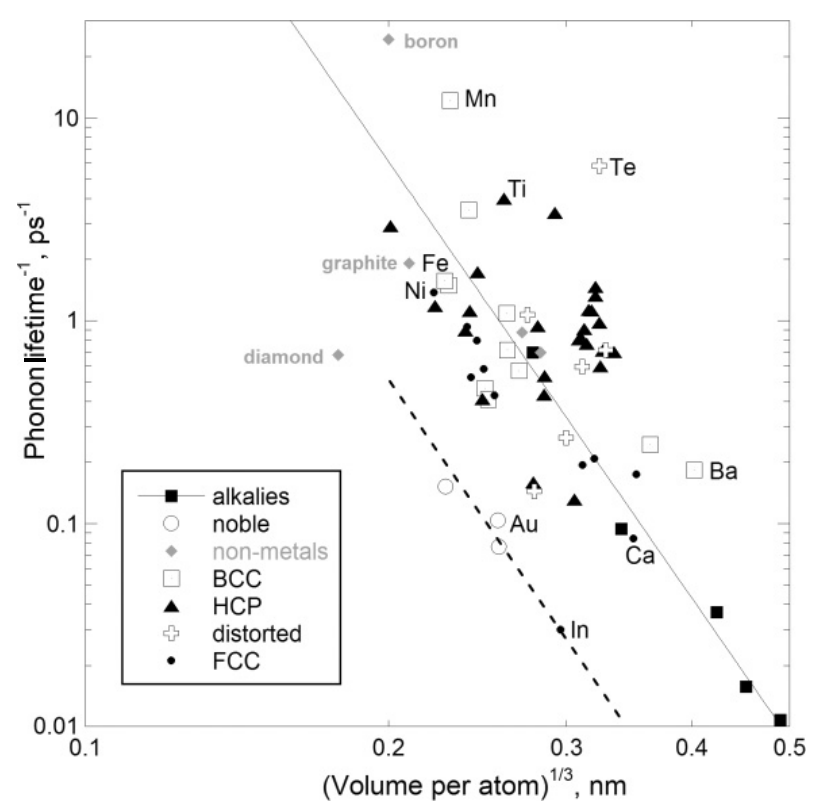

Fig. 23. Comparison of inverse phonon lifetimes with average interatomic distance. Symbols described in the inset. See Fig. 22 and text for data sources. 
bonds being weaker. In this comparison, indium, which has a moderate resistivity, falls on the trend defined by the low resistivity noble metals and purely phononic diamond. Furthermore, calcium and the semiconductors behave like most metals. The trends in lifetimes are largely structural, consistent with a phononic mechanism and Eq. (30a).

Phonon lifetimes of Fig. 23 are compatible with independent measurements of fs to ps for many materials, $\frac{88}{8}$ given that lifetimes strongly depend on frequency (e.g., Ref. 89). For thin films of Si, the lifetime is 1 ps for a frequency of $1 \mathrm{THz}, \stackrel{89}{ }$ which corresponds to $\sim 50 \mathrm{~K}$. Agreement is reasonable, given that our data are on bulk samples, and that lifetimes also depend on film thickness. ${ }^{10,14}$

\subsection{Electronic heat conduction in metals: Mean free paths and lifetimes}

Thermal diffusivities associated with electrons $\left(D_{\text {ele }}\right)$ have large experimental uncertainties $(\sim 25 \%)$, due to the low intensity and rapid onset of the rise. These behaviors result from the low heat capacity and fast speed of electrons. Variations in the reported results (Table 3 ) are outside the uncertainties, showing that $D_{\text {ele }}$ depends on many factors, as discussed in Sec. 4.6 and below. This observed mechanism must be electron-electron scattering (Fig. 3) which is fully consistent with the concept of an electron gas.

Observation of $D_{\text {ele }}$ depends on the rapid rise in $T-t$ curves exceeding the noise. Our measurements best resolve $D_{\text {ele }}$ for metals which have high photon penetration depths and high $C_{\text {ele }}$ but low resistivity samples also provide a rapid rise, although it is generally weak ( $\mathrm{Ag}$ and $\mathrm{Al}$, Fig. 15). Sample length is important because large $L$ attenuates the rapid rise intensity. The diameter of the sample is important, because this provides larger absolute emissions. Increasing the absolute emissions improves $\mathrm{S} / \mathrm{N}$ ratios which permits distinguishing the weak rapid rise from the noise over a wider temperature range. With our apparatus, the rapid rise is below the limit of detection for small samples of most metals. With greater detector sensitivity and better temporal resolution, $D_{\text {ele }}$ should be resolvable in all metals, at moderate lengths, which should delay the signal beyond the pulse without strongly attenuating it. Nonetheless, we were able to obtain data from a wide variety of metals and alloys (Table 3).

All materials studied show similar relationships between $D_{\text {ele }}$ and temperature, generally involving a maximum near $\sim 600 \mathrm{~K}$, as well as a strong discontinuity at the Curie transition. Yet, $D_{\text {ele }}$ is continuous at the few structural transitions probed here (Table 3; Figs. 13-15). In contrast, $D_{\text {lat }}$ decreases with temperature for elements, but increases with temperature for alloys while showing an inflection at the Curie point (Figs. 13-15, or Refs. 18, 57 and 60). Although the structural changes probed here also weakly affect $\overline{D_{\text {lat }}}$, we did not reach conditions where $D_{\text {lat }}$ changes discontinuously with $T$. These graphs show that electronic conduction differs substantially from lattice transport. 
To evaluate $\lambda_{\text {ele }}^{*}$ and $\tau_{\text {ele }}^{*}$, we use $u_{\text {Fermi }}$ for Fermi spheres ${ }^{4,6}$ in Eq. (38) because this permits consistent comparison of our materials. We do not use velocities appropriate to the individual Fermi surfaces (i.e., band structure calculations) because the entire conduction electron population (which has a distribution of velocities) must adjust to the incoming heat (Fig. 3). Although some average velocity is needed, a scheme for averaging, which could be temperature-dependent, requires modeling beyond the scope of this paper. Furthermore, moderate changes involved in using band structure calculations, rather than Fermi spheres, will not alter the trends which are the focus of our discussion. We use constant speed because the $T$ range of our experiments is small compared to $T_{\text {Fermi }}$ which governs electron statistics.

Figure 24 and Table 3 show that electronic mean free paths near $475 \mathrm{~K}$ (the region where they are most accurately determined) are roughly 10 times longer than typical nearest-neighbor bond lengths of $0.22 \mathrm{~nm}$ for the elements. The structurally disordered element $\mathrm{Mn}$ has the shortest $\lambda_{\text {ele }}^{*}$ whereas elements considered to have nearly-free electrons have the longest $\lambda_{\text {ele }}^{*}$. Long $\lambda_{\text {ele }}^{*}$ for $\mathrm{Pb}$ is consistent with very high purity. Mean free paths are considerably shorter in alloys, especially those with extensive cation substitutions and hence great disorder.

Nearly negligible electronic heat transfer for the elements near ambient temperature is consistent with fs-spectroscopy at this temperature, which deduced that lifetimes of electrons excited barely above the Fermi level are infinite near $298 \mathrm{~K}$

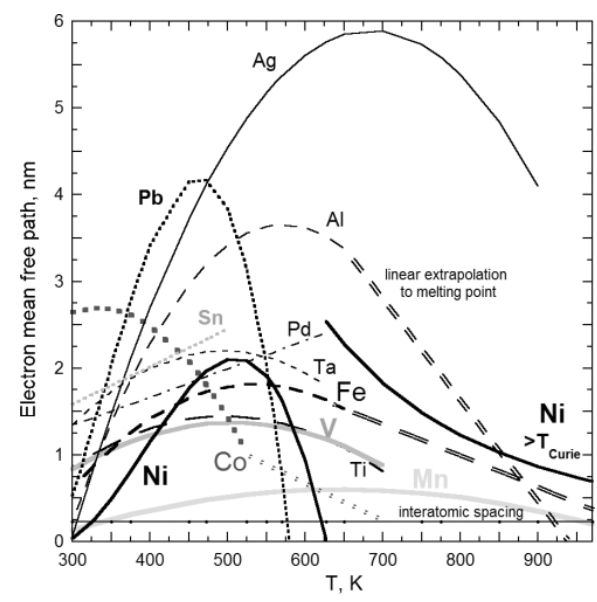

(a)

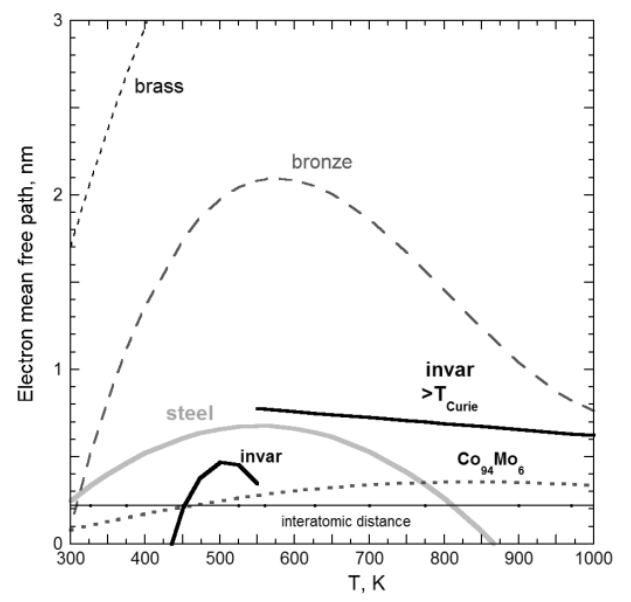

(b)

Fig. 24. Electron-electron mean free paths as a function of temperature calculated from curve fits to $D_{\text {ele }}$ and previous $u_{\text {Fermi }}$ : (a) elements and (b) alloys. In each panel, different line patterns represent different materials as labeled. Double lines are extrapolations from the temperature of measurements to the melting point, where $D_{\text {ele }} \sim 0$. Thicknesses vary for the samples, but are mostly 3-5 mm. The effect of thickness on $D_{\text {ele }}$ was not taken into account, because this is quantified for few of our materials. 
(Fig. 2). Minimally excited electrons which are essentially stable describes our heat transfer experiments. For stability to occur, the interactions must be limited to juxtaposed electrons with similar temperature. Localization is evident in Fig. 24 for both pure metals and alloys. Notably, trends in $\lambda_{\text {ele }}^{*}$ for invar and $\mathrm{Co}_{94} \mathrm{Mo}_{6}$ further indicate that electron-electron scattering does not exist in these materials near room temperature. For these alloys, the rapid rise became evident at temperatures $\sim 100 \mathrm{~K}$ greater than in all our other materials.

Increasing $T$ beyond ambient first increases $\lambda_{\text {ele }}^{*}$ (Fig. 24) which reaches a maximum in the vicinity of $500 \mathrm{~K}$, a rather modest temperature. The subsequent decline at higher $T$ is consistent with fs-spectroscopy, which shows that higher energy electrons are more reactive (less stable). In our experiments, the energy provided by the laser pulse is constant, so that the change in electron behavior with $T$ is connected with the energy (temperature) of the entire population.

The decline in $\lambda_{\text {ele }}^{*}$ at higher $T$ (Fig. 24) is affected by transitions: melting of $\mathrm{Pb}$ causes a rapid drop which is consistent with increasing disorder (premelting effects). Loss of ferromagnetism in $\mathrm{Ni}$ and invar causes an increase, but as this transition is approached from low $T$, thermal transport by electrons is severely impeded, and nearly halted just before the transition. Spin interactions and structural rearrangements clearly impede travel by excited electrons in addition to the shortening of $\lambda_{\text {ele }}^{*}$ associated with higher electron energies. Based on the consistent behavior, we estimate high $T$ trends for $\lambda_{\text {ele }}^{*}$ in $\mathrm{Al}, \mathrm{Co}$, and Fe which have transitions at $T$ not far above our measurements (Fig. 24, double lines).

Length affects $D_{\text {ele }}$ (Fig. 16) and thus will also affect $\lambda_{\text {ele }}^{*}$. Figure 24 is based on $L$ from $1.5 \mathrm{~mm}$ (for $\mathrm{Mn}$ ) to $5.8 \mathrm{~mm}$ (for $\mathrm{Co}$ ). The strong, power-law increase in Fig. 16 would need to be combined with the results in Fig. 24 and Table 3 for a complete description of the mean free path. We did not do so because our dataset

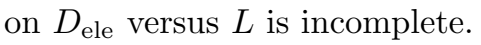

For $\lambda_{\text {ele }}^{*} \sim 1 \mathrm{~nm}$, transport of heat by electrons across $\sim 10 \mathrm{~mm}$ samples means that $\sim 10^{10}$ exchange events follow the thermal excitation of a single electron. For an energy $\delta E$ supplied by the laser pulse to the stream of electrons which cross the sample, before shedding this heat energy to the surroundings as per the second law of thermodynamics, these events involve losses on average of $10^{-10} \delta E$, which supports our deduction of nearly-elastic events during heat transport by electrons. The miniscule amount of energy loss (to other conduction electrons) is nonetheless important because it elevates the temperature of the electron population as the thermal disturbance progresses across the sample.

Lifetimes for heat transport by electron-electron scattering (Fig. 25) are about $1 \mathrm{fs}$ for elements, which is consistent with thermalization times during ultrafast spectroscopy experiments. Alloys have lifetimes resembling that of $\mathrm{Mn}$ which is likewise structurally disordered. Our results on lifetimes are unlike predictions from Landau's Fermi liquid theory ${ }^{4,90}$ whereby $\tau \sim T^{-2}$. Fermi liquid theory is considered to describe reequilibration (cooling) of hot electrons in Ag. ${ }^{27}$ However, warming, 


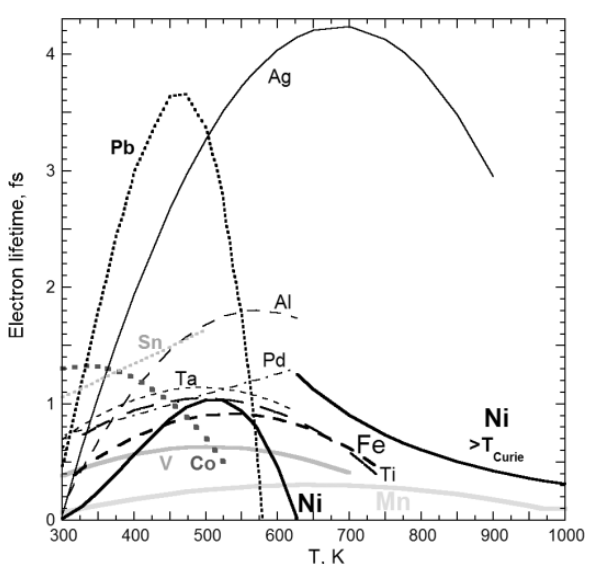

(a)

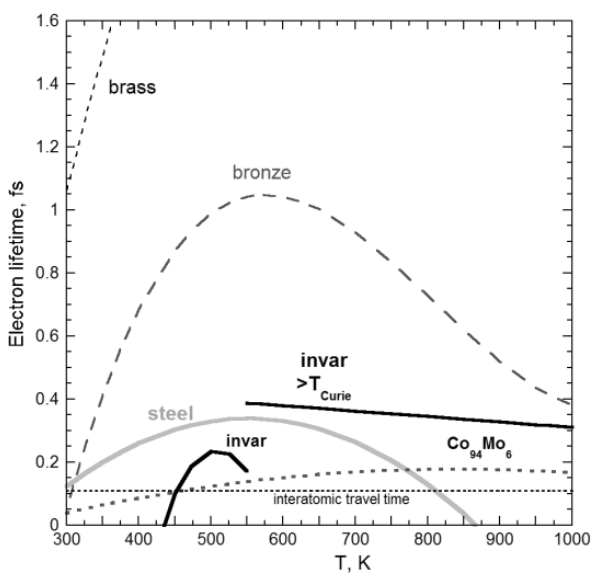

(b)

Fig. 25. Electron-electron lifetimes: (a) elements and (b) alloys. See Fig. 24 for symbols.

which involves changes in the statistical distribution of the entire population, is not addressed by this model.

\subsection{Measured $k_{\mathrm{ele}}, k_{\mathrm{lat}}$, and the Wiedemann-Franz law}

The most incredible aspect of the WF for $k_{\text {ele }}$ [Eq. (5)] is its apparent adherence to $k_{\text {meas }}$ near $298 \mathrm{~K}$ (e.g., Ref. 7; Fig. 1) which actually equals $k_{\text {lat }}$. Three factors contribute. First, the factor of $1 / 3$ in Eq. (5), which is inappropriate to measurements of one-dimensional heat flow in solid, makes $k$ artificially low. Second, Sommerfeld's quantum description [Eq. (5)] gives $L_{S}$ similar to the classical result of $L_{\text {therm }}=3 k_{B}^{2} /\left(2 e^{2}\right)$, after Drude, ${ }^{28}$ because multiplying the thermal velocity by $C_{\text {lat }}$ roughly equals $u_{F} C_{\text {ele }}$ (Refs. 4-7). Third, Eq. (5) utilizes $\tau$ from resistivity measurements. This is especially significant because $\tau$ of an electric field relates to lattice-based heat conduction rather than electronic transport due to the interactions between the vibrating atoms and free electrons (Sec. 1.1). Previous data (Figs. 1 and 4 ), comparison of $D$ to that of electrical insulators, and the data and models in this paper all show that heat transfer measurements at moderate temperatures record a phononic mechanism in metals, except initially and briefly when long lengths are studied.

The above compensating factors in the WF law result in some agreement between measured and predicted $k$ near a single temperature, but show poor agreement with measured $D$, or with $k$, as a function of temperature. The limitation of agreement to only ambient temperature previously evoked concerns (e.g., Ref. 25), since the WF law should be applicable to a wide range of temperatures. The apparent correlation of Eq. (5) with measured results stems not from the 
predictive or descriptive power of this law, but from accidental correspondence of an ambiguous model at a convenient temperature.

More detailed, modern computations of heat transfer share the limitations of the WF law. Modeling heat transfer using a macroscopic approach, as taken here, involves only a limited set of parameters $(k, C$, and $\rho)$, which are directly measurable. However, quantum mechanical descriptions of heat transfer in metals incorporate numerous, unmeasurable quantities which are important and make fitting possible. This difference distinguishes macroscopic from microscopic models, as is well known. 91

The Wiedemann-Franz law cannot be revised to describe electronic heat transport. The mean free path of the thermally excited electrons should not be related to resistivity because the latter property centers on interactions of electrons with phonons that do not involve thermally-induced changes in energy states. Direct measurement of $D_{\text {ele }}$ shows a very complicated dependence on temperature that is unlike existing models (e.g., Refs. 3, 8, and 90). New models are needed which consider perturbation of the energy levels of all conduction electrons during heat transport.

From Table $3, k_{\text {ele }}$ is many times the literature values for $k_{\text {lat }}$. Although it seems counterintuitive that the $T-t$ curve belonging to the more conductive mechanism would have been overlooked for so long, this omission follows the assumption that conductivities sum at high temperature, Eq. (10). Our experiments and models show that even in limiting cases with similar diffusivities, conductivities do not simply sum.

For metallic behavior, diffusivities are not similar. In this case, heat is transported by two approximately independent mechanisms at much different rates. The electronic mechanism has an extremely high conductivity, but transports very little heat. Lattice mechanisms transport heat slowly, but in quantity, resulting in Eq. (30a). Essentially, the importance of the mechanism is not determined by its conductivity, but by its ability to absorb energy, which stems from energy conservation. The small size of the electronic component has caused it to be repeatedly overlooked; this omission was masked by the incorrect interpretation that the single observed thermal curve was the combined result of both phenomena, and by the existence of spark noise in early experiments.

Our measurements of $D_{\text {lat }}$ and $D_{\text {ele }}$ allow us to reconsider basic assumptions involving heat transfer, and heat transfer of electrons in particular. We propose that the lifetime associated with our measurements of $D_{\text {ele }}$ is governed mainly by electron-electron collisions, because such events will transfer heat across $\sim 10 \mathrm{~mm}$ samples at the Fermi velocity over small scales. Electron-phonon collisions, whereby the excess energy of a thermally-activated electron is shed to a phonon, describe the slower process of thermal reequilibration of the electrons. 


\section{Conclusions}

Laser-flash measurements above ambient temperature utilizing unusually long sample lengths concomitantly with short data collection times show that two independent diffusive processes exist in various metals and alloys, consistent with previous inferences that both electrons and phonons transport heat in such materials. $\frac{1,2,18}{}$ The unique characteristics of this method (its simplicity, robustness, and ability to examine transient phenomena $)^{38,39}$ permit distinguishing these two processes. The faster process must be electronic, based on known carrier speeds, because diffusive or ballistic radiative transfer is ruled out for several reasons. The electronic signal height is weak and temperature-independent, consistent with data and theory on electronic heat capacity. Phonons are the main carrier of heat, consistent with their large heat capacity. We refute electrons as the cause of the main signal in LFA based on available values of $D$ (or of $D$ from $k$ ) and of $u_{\text {Fermi }}$. Our associations of electron-electron scattering with very brief transient heating and of phonon scattering with quasi-steady-state heat transport are confirmed by inferred $\lambda$ and $\tau$ values. Phononic mean free paths and lifetimes calculated from $D_{\text {lat }}=D_{\text {total }}$ depend on structure, with $D$ values being like those of purely phononic diamond and mostly phononic semiconductors, whereas our electronic mean free paths and lifetimes are consistent with previous, independent fs-spectroscopy $\frac{14}{14}$ of various processes in metals and pnictides. Secondary evidence exists in the simple response of $D_{\text {ele }}$ to temperature, and in the need for relatively high $C_{\text {ele }}$ and photon penetration in order to resolve $D_{\text {ele }}$ above noise in the LFA signals.

Modern models of microscopic heat conduction are based on the kinetic theory of gases, and contain errors because this historic model was derived assuming steadystate conditions and a small thermal gradient. Such conditions involve a negligible amount of heat moving randomly, and do not describe heat transfer experiments in solids which involve a unidirectional thermal gradient of various magnitudes, and independent flow of mass and heat. Assuming steady-state conditions, low$T$ gradients stemmed from the need to confirm theory with measurements made under conditions that circumvented convection. This disconnect with solid behavior was compounded in analyzing metals by simply summing electronic and lattice thermal conductivities which only conserves energy under very restricted conditions (Sec. 5). Our adiabatic model shows that phonons control heat transport in metals above $\sim 20 \mathrm{~K}$ [i.e., $k=k_{\text {lat }}$ : Eq. (30)], corroborating our experimental results. Due to compensating errors (i.e., $u_{\text {lat }} C_{\text {lat }}=u_{\text {Fermi }} C_{\text {ele }}$ ), the Wiedemann-Franz law correlates not with the electronic processes in a metal, but with heat transfer through the lattice, contributing to a systematic misunderstanding regarding the mechanisms inherent to heat transfer.

Our findings and revised formulations have the potential to improve our understanding of microscopic phenomena involving interactions of electrons with other electrons and/or phonons, such as metal-insulator transitions. Applications include ascertaining the thermal conductivity of nanomaterials and temperatures inside 
planets. Our findings have practical implications for the design of heat exchangers and thermal interfaces. The improved understanding of material behavior, including transient events, contributed by this paper will eventually result in improvements to heating and cooling systems, heat engines, transformers, and integrated circuits.

\section{Acknowledgments}

Support was provided by NSF EAR-0757841, 1321857 and 1524495. We thank T. Hardt and R. E. Criss (WU), J. M. Branlund (SWIC), and T. Kuech (University of Wisconsin) for help and input. We thank Dr. Bimal Kad (University of California San Diego) for providing the CoMo sample. Any opinions, findings, and conclusions, or recommendations expressed in this material are those of the authors and do not necessarily reflect the views of the NSF.

\section{References}

1. J. Koenigsberger, Phys. Z. 8, 237 (1907).

2. P. J. Klemens, in Low Temperature Physics I, eds. J. G. Daunt et al., Encyclopedia of Physics Series, Vol. 3 (Springer, Berlin, 1956), p. 198.

3. C. Uher, in Thermal Conductivity, ed. T. M. Tritt (Kluwer Academic, New York, 2004), p. 21.

4. N. W. Ashcroft and N. D. Mermin, Solid State Physics (Holt, Rinehart and Winston, New York, 1976), pp. 20-24, 30-61, 140-145, 345-351.

5. P. G. Klemens and R. K. Williams, Int. Met. Rev. 31, 197 (1986).

6. G. Burns, Solid State Physics (Academic Press, San Diego, 1990), pp. 187-221, 433-435.

7. C. Kittel, Introduction to Solid State Physics, Fourth edn. (John Wiley \& Sons, New York, 1971), pp. 224-226, 239-265.

8. J. M. Ziman, Electrons and Phonons: The Theory of Transport Phenomena in Solid (Clarendon Press, Oxford, 1962); Principles of the Theory of Solids (Cambridge University Press, 1972).

9. H. E. Elsayed-Ali et al., Phys. Rev. Lett. 58, 1212 (1987); R. W. Schoenlein et al., Phys. Rev. Lett. 58, 680 (1987).

10. M. Aeschlimann et al., Appl. Phys. A 71, 485 (2000).

11. A. Patz et al., Nat. Commun. 5, 3229 (2014), doi:10.1038/ncomms4229.

12. E. Beaurepaire et al., Phys. Rev. Lett. 76, 4250 (1996).

13. J.-Y. Bigot, M. Vomir and E. Beaurepaire, Nat. Phys. 5, 515 (2009).

14. M. Bauer, A. Marienfeld and M. Aeschlimann, Prog. Surf. Sci. 90, 319 (2015).

15. P. B. Corkum, F. Brunel and N. K. Sherman, Phys. Rev. Lett. 61, 2886 (1988).

16. S. D. Brorson et al., Phys. Rev. Lett. 64, 2172 (1990); H. E. Elsyed-Ali et al., Phys. Rev. B 43, 4488 (1991).

17. V. P. Zhukov and E. V. Chulkov, Phys.-Usp. 52, 105 (2009).

18. C. Y. Ho et al., J. Phys. Chem. Ref. Data 7, 959 (1978).

19. R. K. Williams et al., J. Appl. Phys. 52, 5167 (1981).

20. A. Sommerfeld, Z. Phys. 47, 1 (1928).

21. E. S. R. Gopal, Specific Heats at Low Temperatures (Plenum Press, New York, 1966); M. H. Aven et al., Phys. Rev. 102, 1263 (1956).

22. Y. S. Touloukian et al., Thermal Diffusivity (IFI/Plenum Press, New York, 1973). 
23. W. F. Gale and T. C. Totemeier, Smithells Metals Reference Book, 8th edn. (Elsevier, Oxford, 2004).

24. Element Collection, Inc., Thermal conductivity of the elements (2014), http://www. periodictable.com/Properties/A/ThermalConductivity.v.html. [The information was crossed checked against I. S. Grigoriev and E. Z. Meilikhov, Handbook of Physical Quantities (CRC Press, Boca Raton, 1997); Y. S. Touloukian et al., Thermal Conductivity: Metallic Elements and Alloys (IFI/Plenum Press, New York, 1970); and Refs. 18, 22, and 23].

25. J. G. Hust and L. L. Sparks, NBS Technical Note 634 (U.S. Government Printing Office, Washington, DC, 1973).

26. V. V. Kruglyak et al., Phys. Rev. B 71, 233104 (2005).

27. M. Bauer and M. Aeschlimann, J. Electron Spectrosc. Relat. Phenom. 124, 225 (2002).

28. P. Drude, Ann. Phys. 1, 566 (1900); 3, 369 (1900).

29. M. K. Transtrum et al., J. Chem. Phys. 143, 010901 (2015).

30. C. A. Paddock and G. L. Easley, J. Appl. Phys. 60, 285 (1986); K. Hatori et al., Rev. Sci. Instrum. 76, 114901 (2005); T. Baba, in First Int. Symp. Thermal Design and Thermophysical Property for Electronics, Tsukaba, Japan (2008); G. Matsui and H. Kato, Rev. Sci. Instrum. 83, 034905 (2011).

31. A. J. Schmidt, X. Chen and G. Chen, Rev. Sci. Instrum. 79, 114902 (2008).

32. D. G. Cahill and R. O. Pohl, Phys. Rev. B 35, 4067 (1987).

33. A. M. Hofmeister, J. J. Dong and J. M. Branlund, J. Appl. Phys. 115, 163517 (2014).

34. M. Pertermann et al., Contrib. Mineral. Petrol. 155, 689 (2008); A. M. Hofmeister et al., Am. Mineral. 99, 564 (2014).

35. H. S. Carslaw and J. C. Jaeger, Conduction of Heat in Solids, 2nd edn. (Oxford University Press, New York, 1959), pp. 92-114.

36. A. M. Hofmeister, Phys. Earth Planet. Inter. 180, 138 (2010).

37. W. J. Parker et al., J. Appl. Phys. 32, 1679 (1961); R. L. Rudkin, R. J. Jenkins and W. J. Parker, Rev. Sci. Instrum. 33, 21 (1962).

38. L. Vozár and W. Hohenauer, Int. J. Thermophys. 26, 1899 (2005).

39. L. Vozár and W. Hohenauer, High Temp.-High Press. 35-36, 253 (2003/2004).

40. M. A. Ordal et al., Appl. Opt. 24, 4493 (1985).

41. D. W. Lynch and W. R. Hunter, in Handbook of Optical Constants of Solids II, ed. E. D. Palik (Academic Press, Orlando, 1991), pp. 341-377; M. R. Querry, Contractor report for DOD (1985), http://oai.dtic.mil/oai/oai?verb=getRecord\&metadataPrefix= html\&identifier $=$ ADA 158623 .

42. W. Meissner, Ann. Phys. 47, 1001 (1915).

43. E. Gruneisen and E. Goens, Z. Phys. 44, 615 (1927).

44. C. C. Bidwell, Phys. Rev. 32, 311 (1928).

45. J. M. Cork, Heat (Chapman and Hall, London, 1933); R. E. B. Makinson, Math. Proc. Camb. Philos. Soc. 34, 474 (1938).

46. X. Zheng et al., Acta Mater. 55, 5177 (2007).

47. N. Wakeham et al., Nat. Commun. 2, 396 (2011), doi:10.1038/ncomms1406.

48. B. Sundqvist, Solid State Commun. 37, 289 (1981); in High Pressure in Research and Industry, eds. C. M. Backman, T. Johannison and L. Tegnér (Arkitektkopia, Uppsala, 1982), p. 432.

49. X. Tang and J. J. Dong, Phys. Earth Planet. Inter. 174, 33 (2009); Proc. Natl. Acad. Sci. 107, 4539 (2010).

50. C. T. Ewing et al., in Liquid Metals Technology - Part 1, Chemical Engineering Progress Symposium Series, Vol. 53 (American Institute of Chemical Engineers, 1957), pp. 19-24. 
51. C. S. Smith and E. W. Palmer, Trans. AIME 117, 225 (1935).

52. R. W. Powell, Int. J. Heat Mass Transf. 8, 1033 (1965).

53. K. D. Maglić and R. E. Taylor, in Compendium of Thermophysical Property Measurement Methods, eds. K. D. Maglić, A. Cezairliyan and V. E. Peletsky, Vol. 2 (Plenum Press, New York, 1992), p. 281.

54. D. R. Cowan, J. Appl. Phys. 34, 926 (1963); J. A. Cape and G. W. Lehman, J. Appl. Phys. 34, 1909 (1963).

55. H. Mehling et al., Int. J. Thermophys. 19, 941 (1998); J. Blumm et al., High Temp.-High Press. 29, 555 (1997); R. Hofmann et al., High Temp.-High Press. 29, 703 (1997).

56. J. Blumm and S. Lemarchand, High Temp.-High Press. 34, 523 (2002); J. Blumm and J. Opfermann, High Temp.-High Press. 34, 515 (2002).

57. B. J. Monaghan and P. N. Quested, ISIJ Int. 41, 1524 (2001).

58. J. B. Henderson, L. Hagemann and J. Blumm, Development of SRM 8420 series electrolytic iron as a thermal diffusivity standard, Netzsch Applications Laboratory Thermophysical Properties Section Report No. I-9E (1998), pp. 1-11; J. B. Henderson et al., Int. J. Thermophys. 19, 1647 (1998).

59. J. G. Hust and A. B. Lankford, Update of thermal conductivity and electrical resistivity of electrolytic iron, tungsten, and stainless steel, National Bureau of Standards Special Publication 260-90, US DoC, USA (1984), pp. 1-71.

60. V. I. Gorbatov et al., High Temp. 50, 292 (2012).

61. A. M. Hofmeister, Phys. Chem, Mineral. 33, 45 (2006); A. M. Hofmeister, J. Appl. Phys. 107, 103532 (2010); M. Perterman and A. M. Hofmeister, Am. Mineral. 91, 1474 (2006); Y. Xu and A. M. Hofmeister, J. Appl. Phys. 109, 033516 (2011).

62. J. M. Branlund and A. M. Hofmeister, Am. Mineral. 93, 1620 (2008).

63. V. F. Buchwald, Handbook of Iron Meterorites: Their History, Distribution, Composition and Structure (University of California Press, 1975) [see Vol. 3, p. 917 for specifics on the North Chile locality].

64. P. J. Webster, Contemp. Phys. 10, 599 (1969).

65. C. Y. Ho et al., J. Phys. Chem. Ref. Data 12, 183 (1983).

66. H. Bräuer, L. Dusza and B. Schulz, Interceram 41, 489 (1992) [also see https://www.netzsch-thermal-analysis.com/us/products-solutions/ thermal-diffusivity-conductivity/lfa-427/].

67. A. M. Hofmeister and A. G. Whittington, J. Non-Cryst. Solids 358, 1072 (2012).

68. A. M. Hofmeister, Phys. Chem. Mineral. 41, 361 (2014).

69. R. A. Friedel and G. L. Carlson, J. Phys. Chem. 75, 1149 (1971).

70. A. M. Hofmeister and E. M. Criss, Thermal diffusivity of the Fe-Ni binary including meteorites and melts: Mechanisms of heat transport in metals and implications for planetary cores. To be submitted to Physics of the Earth and Planetary Interiors.

71. H. M. Ledbetter and R. P. Reed, J. Phys. Chem. Ref. Data 2, 531 (1973).

72. I. Simon, J. Opt. Soc. Am. 12, 547 (1951); V. Lucarini et al., Kramers-Kronig Relations in Optical Materials Research (Springer, Heidelberg, 2005).

73. F. Wooten, Optical Properties of Solids (Academic Press, New York, 1972).

74. R. Siegel and J. R. Howell, Thermal Radiation Heat Transfer (McGraw-Hill, New York, 1972).

75. A. D. Rakić, Appl. Opt. 34, 4755 (1995).

76. F. Reif, Fundamentals of Statistical and Thermal Physics (McGraw-Hill, New York, 1965), pp. 471-483.

77. D. G. Cahill, Rev. Sci. Instrum. 61, 802 (1990).

78. A. M. Hofmeister, M. Pertermann and J. M. Branlund, in Thermal Conductivity of the Earth: Mineral Physics, ed. G. Schubert, Treatise on Geophysics, Vol. 2 (Elsevier, 
The Netherlands, 2007), p. 543.

79. T. H. K. Barron, Philos. Mag. 46, 720 (1955); R. S. Krishnan, R. Srinivasan and S. Devanarayanan, Thermal Expansion of Crystals (Pergamon Press, New York, 1979).

80. E. Grüneisen, Ann. Phys. 39, 257 (1912); Handb. Phys. 10, 1 (1926) [see also the NASA translation of the article, Publication No. RE2-18-59W (1959)].

81. A. M. Hofmeister and H. K. Mao, Proc. Natl. Acad. Sci. 99, 559 (2002).

82. R. T. Beyer and S. V. Letcher, Physical Ultrasonics (Academic Press, New York, 1969).

83. C. Stassis et al., Phys. Rev. B 18, 2632 (1978).

84. J. P. Jan and H. L. Skriver, J. Phys. F, Met. Phys. 11, 805 (1981).

85. D. Y. Petrovykh et al., Appl. Phys. Lett. 73, 3459 (1998); P. B. Allen, in Quantum Theory of Real Materials, eds. J. R. Chelikowsky and S. G. Louie (Kluwer Academic, Boston, 1996), p. 219.

86. J. M. Branlund and A. M. Hofmeister, Am. Mineral. 97, 1145 (2012).

87. C. K. Sun et al., Phys. Rev. B 50, 15337 (1994) [see also Ref. 12 and references therein].

88. W. E. Bron, Ultrasonic Processes in Condensed Matter (Springer, New York, 2012).

89. J. Cuffe et al., Phys. Rev. Lett. 110, 0950013 (2013).

90. L. D. Landau, Sov. Phys.-JETP 3, 920 (1957); 5, 101 (1957); 8, 70 (1959).

91. M. W. Zemansky and R. H. Dittman, Heat and Thermodynamics, 6th edn. (McGrawHill, New York, 1981). 